\title{
Interaction of Risk Factors, Comorbidities, and Comedications with Ischemia/Reperfusion Injury and Cardioprotection by Preconditioning, Postconditioning, and Remote Conditioning
}

\author{
Péter Ferdinandy, Derek J. Hausenloy, Gerd Heusch, Gary F. Baxter, and Rainer Schulz
}

Department of Pharmacology and Pharmacotherapy, Semmelweis University, Budapest, Hungary (P.F.); Cardiovascular Research Group, Department of Biochemistry, University of Szeged, Szeged and Pharmahungary Group, Szeged, Hungary (P.F.); The Hatter Cardiovascular Institute, University College London, London, United Kingdom (D.J.H.); Institute for Pathophysiology, University of Essen Medical School, Essen, Germany (G.H.); Division of Pharmacology, Cardiff School of Pharmacy and Pharmaceutical Sciences, Cardiff University, Cardiff, United Kingdom (G.F.B.); and Institute of Physiology, Justus-Liebig University, Giessen, Germany (R.S.)

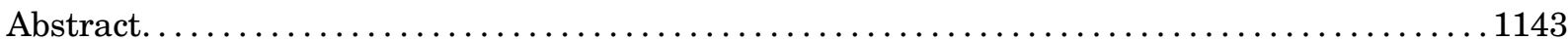

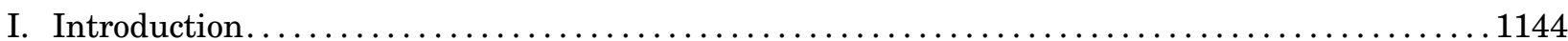

II. Experimental Approaches to Cardioprotection. . . . . . . . . . . . . . . . . . . . . . . . . . . . . 1145

A. Cardioprotection through Preconditioning ................................. 1145

1. Mitochondria and Preconditioning.................................... 1145

a. The mitochondrial permeability transition pore and preconditioning. $\ldots \ldots \ldots \ldots . \ldots 1145$

b. Mitochondrial connexin- 43 and preconditioning. ......................... 1146

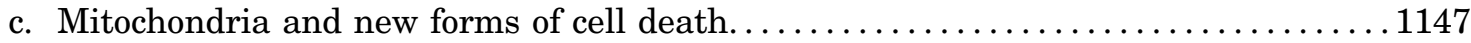

d. Mitochondrial dynamics and cardioprotection. .......................... 1147

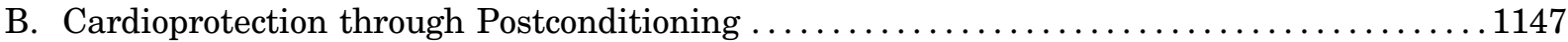

1. Autacoid Mediators of Postconditioning................................. 1148

2. Delaying the Correction of $\mathrm{pH}$ at Reperfusion. ............................. 1148

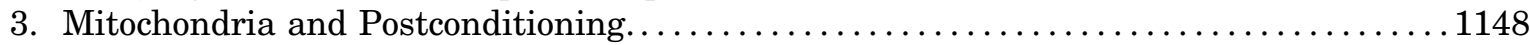

C. Cardioprotection through Pharmacologic Conditioning ........................ 1148

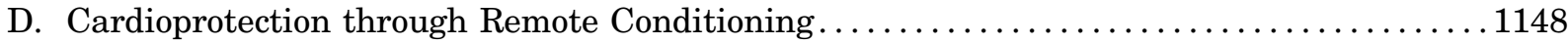

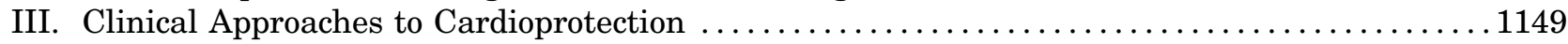

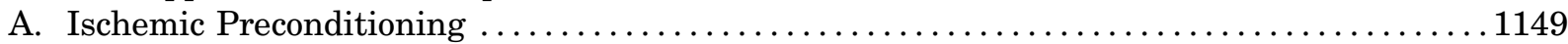

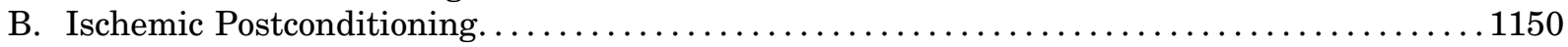

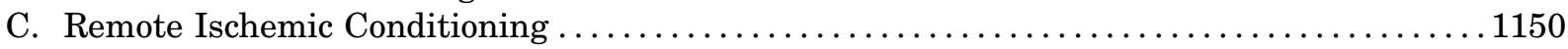

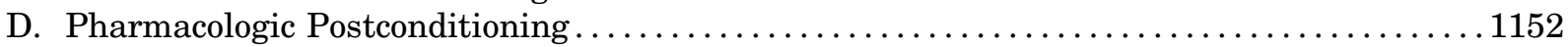

IV. Effects of Major Risk Factors on Ischemia/Reperfusion Injury and Cardioprotective

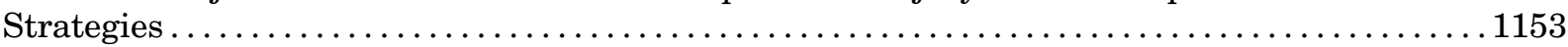

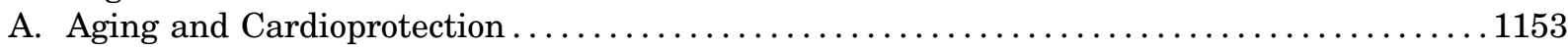

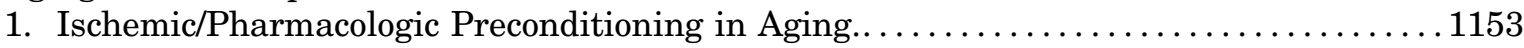

a. Effect of aging on cardioprotective signaling.......................... 1153

i. Cytosolic Signaling. . . . . . . . . . . . . . . . . . . . . . . . . . . . . . . . . 1153

ii. Mitochondria. .............................................. 1154

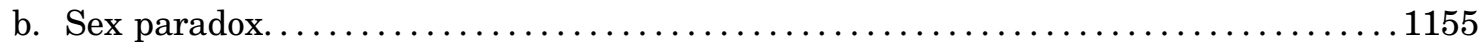

c. Delayed preconditioning (second window of protection) $\ldots \ldots \ldots \ldots \ldots \ldots \ldots \ldots \ldots \ldots \ldots$

This work was supported by grants from the British Heart Foundation [Grant FS/10/039/28270] and the RoseTrees Trust (to D.J.H.); the German Research Foundation [Grants DFG Schu 843/7-1; 843/7-2; 843/9-1] (to R.S.) and [Grants He 1320/18-1,3] (to G.H.); the National Research Fund of Hungary [Grants ANN 107803, K 109737) (to P.F.); the European Foundation for the Study of Diabetes (to P.F. and R.S.). D.H. is supported by the National Institute for Health Research University College London Hospitals Biomedical Research Centre. P.F. is a Szentágothai fellow of the National Excellence Program of Hungary [Grant TAMOP 4.2.4.A/2-11-1-2012-0001].

Address correspondence to: Dr. Peter Ferdinandy, Department of Pharmacology and Pharmacotherapy, Semmelweis University, Nagyvárad tér 4, Budapest, H-1089, Hungary. E-mail: peter.ferdinandy@pharmahungary.com

dx.doi.org/10.1124/pr.113.008300. 
2. Aging and Ischemic and Pharmacologic Postconditioning. .................. 1155

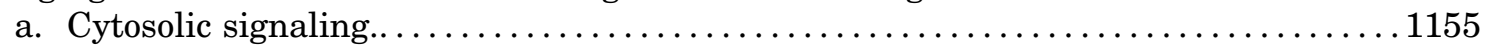

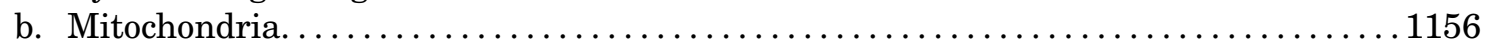

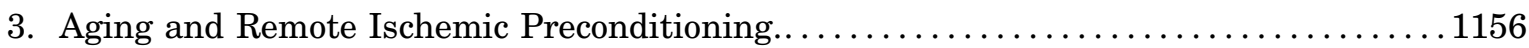

B. Hypertension, Cardiac Hypertrophy, and Remodeling. ........................ 1156

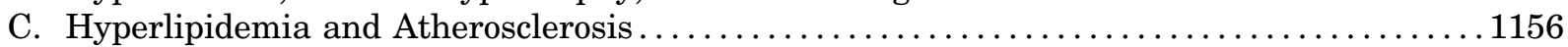

1. Ischemia/Reperfusion Injury, Ischemic Pre-, Post-, and Remote Conditioning in

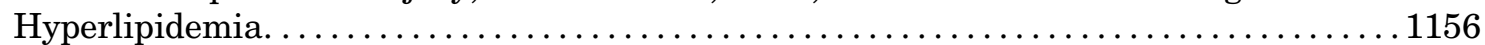

2. Pharmacologic Cardioprotection in Hyperlipidemia: Implications for Target

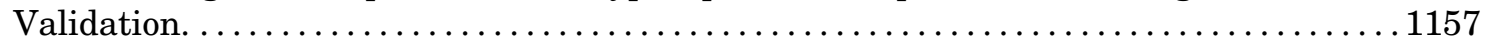

3. Effect of Hyperlipidemia on Cardioprotective Cellular Mechanisms................. 1157

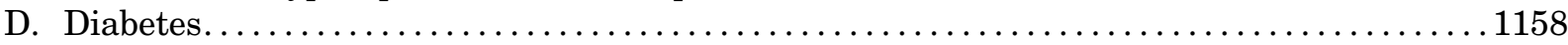

1. Ischemia/Reperfusion Injury in Diabetes. ............................. 1158

2. Cardioprotection by Preconditioning in Diabetes ......................... 1158

a. Mechanisms contributing to resistance of the diabetic heart to preconditioning.....1158

b. Restoring myocardial sensitivity to preconditioning in the presence of diabetes. ...1159

3. Cardioprotection by Postconditioning in Diabetes and Metabolic Syndrome...........1160

4. Cardioprotection by Remote Ischemic Conditioning in Diabetes. . . . . . . . . . . . 1161

E. Kidney Failure and Uremia .......................................... 1162

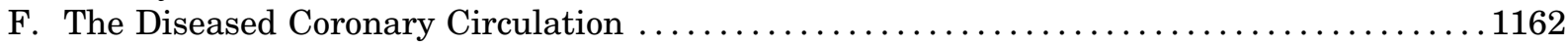

1. Epicardial Coronary Arteries......................................... 1162

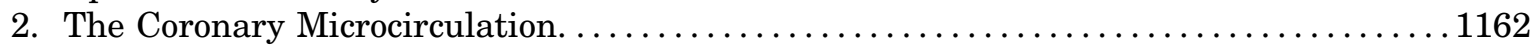

V. Effects of Concomitant Medications Used to Treat Risk Factors and Comorbidities on

Cardioprotection: Hidden Cardiotoxicity?.................................... 1163

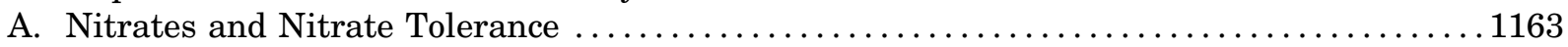

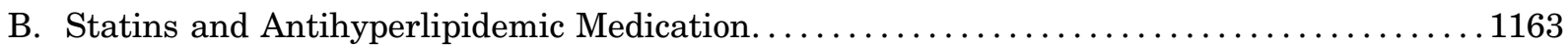

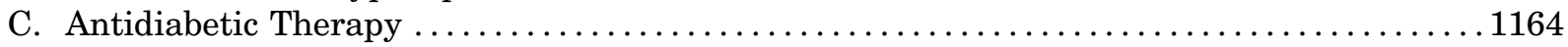

1. Antidiabetic Therapy and ATP-Sensitive Potassium Channels. . . . . . . . . . . . 1165

2. Antidiabetic Therapy with Mixed Actions on Cardioprotection. . . . . . . . . . . . . 1165

D. $\beta$-Adrenoceptor Antagonists. ........................................... 1165

E. Angiotensin-Converting Enzyme Inhibitors/Angiotensin II Receptor Type 1 Receptor

Antagonists ....................................................... 1166

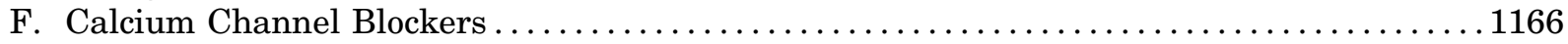

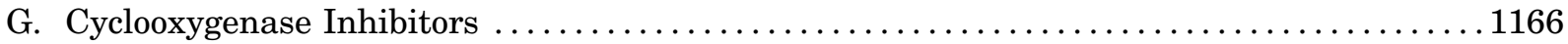

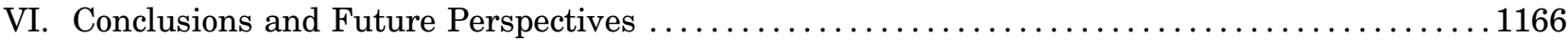

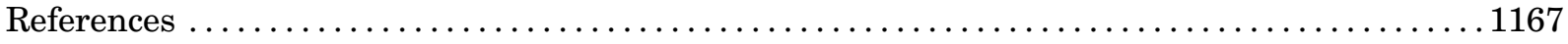

\begin{abstract}
Pre-, post-, and remote conditioning of the myocardium are well described adaptive responses that markedly enhance the ability of the heart to withstand a prolonged ischemia/reperfusion insult and provide therapeutic paradigms for cardioprotection. Nevertheless, more than 25 years after the discovery of ischemic preconditioning, we still do not have established cardioprotective drugs on the market. Most experimental studies on cardioprotection are still undertaken in animal models, in which ischemia/reperfusion is imposed in the absence of cardiovascular risk factors. However, ischemic heart disease in humans is a complex disorder caused by, or associated with, cardiovascular
\end{abstract}

risk factors and comorbidities, including hypertension, hyperlipidemia, diabetes, insulin resistance, heart failure, altered coronary circulation, and aging. These risk factors induce fundamental alterations in cellular signaling cascades that affect the development of ischemia/ reperfusion injury per se and responses to cardioprotective interventions. Moreover, some of the medications used to treat these risk factors, including statins, nitrates, and antidiabetic drugs, may impact cardioprotection by modifying cellular signaling. The aim of this article is to review the recent evidence that cardiovascular risk factors and their medication may modify the response to cardioprotective interventions. We emphasize the

ABBREVIATIONS: ACE, angiotensin-converting enzyme; AMPK, adenosine monophosphate-activated kinase; AT1, angiotensin II receptor type 1; CCB, L-type calcium channel blocker; COX-2, cyclo-oxygenase-2; Cx43, connexin 43; Drp1, dynamin-related protein; eNOS, endothelial NO synthase; ERK, extracellular signal-regulated kinase; GLP-1, glucagon-like peptide-1; GSK-3 $\beta$, glycogen synthase-3 $\beta$; K ATP $_{\text {, ATP-sensitive }}$ potassium channel; MI, myocardial infarction; MMP, matrix metalloproteinase; MPTP, mitochondrial permeability transition pore; PCI, percutaneous coronary intervention; PI3K, phosphatidylinositol 3-kinase; PKA, cAMP-dependent protein kinase; PKC, protein kinase C; PKG, cGMP-dependent protein kinase; PTEN, phosphatase and tensin homologue; RISK, reperfusion injury salvage kinase; ROS, reactive oxygen species; SAFE, survivor activating factor enhancement; Sirt1, sirtuin deacetylase 1; STEMI, ST-segment elevated MI; STAT3, signal transducer and activator of transcription-3; TNF $\alpha$, tumor necrosis factor $\alpha$. 
critical need to take into account the presence of cardiovascular risk factors and concomitant medications when designing preclinical studies for the identification and validation of cardioprotective drug targets and clinical studies. This will hopefully maximize the success rate of developing rational approaches to effective cardioprotective therapies for the majority of patients with multiple risk factors.

\section{Introduction}

Ischemic heart disease is one of the leading causes of death and disability in the industrialized societies. Effective treatment of acute myocardial infarction (MI) is based on procedures that promote the return of blood flow to the ischemic zone of the myocardium, i.e., reperfusion therapy. Reperfusion, however, may lead to further irreversible myocardial cell death, termed lethal myocardial reperfusion injury. Currently, there is no effective therapy for combined ischemia/ reperfusion injury on the market, and routine pharmacologic agents do not salvage the ischemic/reperfused myocardium. Therefore, the development of cardioprotective agents to limit the extent of infarcted tissue caused by ischemia/reperfusion injury is of great clinical importance.

Earlier pharmacologic approaches to attenuate the consequences of ischemia/reperfusion injury were of limited experimental efficacy or failed to translate into useful clinical treatments. However, in the last three decades, the heart has been shown to possess a remarkable ability to adapt to ischemia/reperfusion stress, and this molecular plasticity of the heart in ischemia/ reperfusion has been the focus of intense research in the hope that the underlying mechanisms may be amenable to therapeutic exploitation. Ischemic preconditioning, postconditioning, and remote conditioning of myocardium are well described adaptive responses in which there is brief exposure to ischemia/reperfusion before sustained ischemia (preconditioning), or at the immediate onset of reperfusion (postconditioning), or in a remote tissue before, during, or at reperfusion after sustained myocardial ischemia (remote conditioning). All forms of conditioning markedly enhance the ability of the heart to withstand a prolonged ischemic insult (Fig. 1). The discovery of these endogenous cardioprotective mechanisms has encouraged the exploration of new ways to protect the ischemic/reperfused myocardium and has amplified our knowledge of the molecular basis of cell injury and survival mechanisms during ischemia/reperfusion.

Ischemic heart disease develops as a consequence of a number of etiologic risk factors predisposing to atherosclerosis development; it always coexists with other systemic disease states. These predisposing and/ or coexisting conditions include systemic arterial hypertension with related left ventricular hypertrophy and disturbed coronary circulation (i.e., hypertensive heart disease); metabolic diseases, such as hyperlipidemia, diabetes mellitus, obesity, insulin resistance, uremia; and heart failure (Heusch et al., 2014). In addition, aging is a major risk factor predisposing to the development of ischemic heart disease. These risk factors and coexisting conditions exert multiple biochemical effects on the heart that affect the development of ischemia/ reperfusion injury per se and interfere with responses to cardioprotective interventions. Moreover, routine drug therapies for these conditions, e.g., antihyperlipidemic, antidiabetic, antihypertensive, antianginal, and antiplatelet drugs, as well as drugs indicated for noncardiovascular diseases, may also interfere with cardioprotective interventions.

Since the original observations of the loss of preconditioning in hyperlipidemic rodents (Szilvassy et al., 1995; Ferdinandy et al., 1997), it has been well established that many of the cardiovascular risk factors may interfere with cardioprotection by conditioning strategies (see our earlier reviews: Ferdinandy et al., 1998, 2007; Ferdinandy, 2003; Ovize et al., 2010). Nevertheless, most experimental studies on cardioprotection are still undertaken in juvenile healthy animal models, in which ischemia/reperfusion is imposed in the absence of the classical risk factors for cardiovascular disease. This has contributed, at least in part, to the slow progress of translation of preclinical results to clinical therapy. Although some conditioning treatments in humans have shown promising results, other studies have shown no cardioprotective effect of conditioning in patients with acute MI (Heusch, 2013). Therefore, the development of rational therapeutic approaches to protect the ischemic heart requires preclinical studies that examine cardioprotection specifically in relation to cardiovascular risk factors and their medications. Moreover, to avoid unexpected ischemiarelated safety problems, the interaction of drugs with endogenous cardioprotective mechanisms must be tested during preclinical and clinical phases of drug development as well as in postmarketing clinical studies.

The aim of this review is to update our previous review (Ferdinandy et al., 2007) on the effects of risk factors on ischemia/reperfusion injury and cardioprotection and to emphasize the ongoing critical need for preclinical studies that model the presence of risk factors and their pharmacologic treatments. Such studies are required for the proper validation of molecular targets for cardioprotection, thereby maximizing the chances of success for translation of cardioprotection into the clinical arena and for the benefit of the majority of ischemic heart disease patients who have multiple risk factors and associated medications. Furthermore, we highlight that routine medications for cardiovascular and other diseases may show undesirable effects on endogenous cardioprotective 


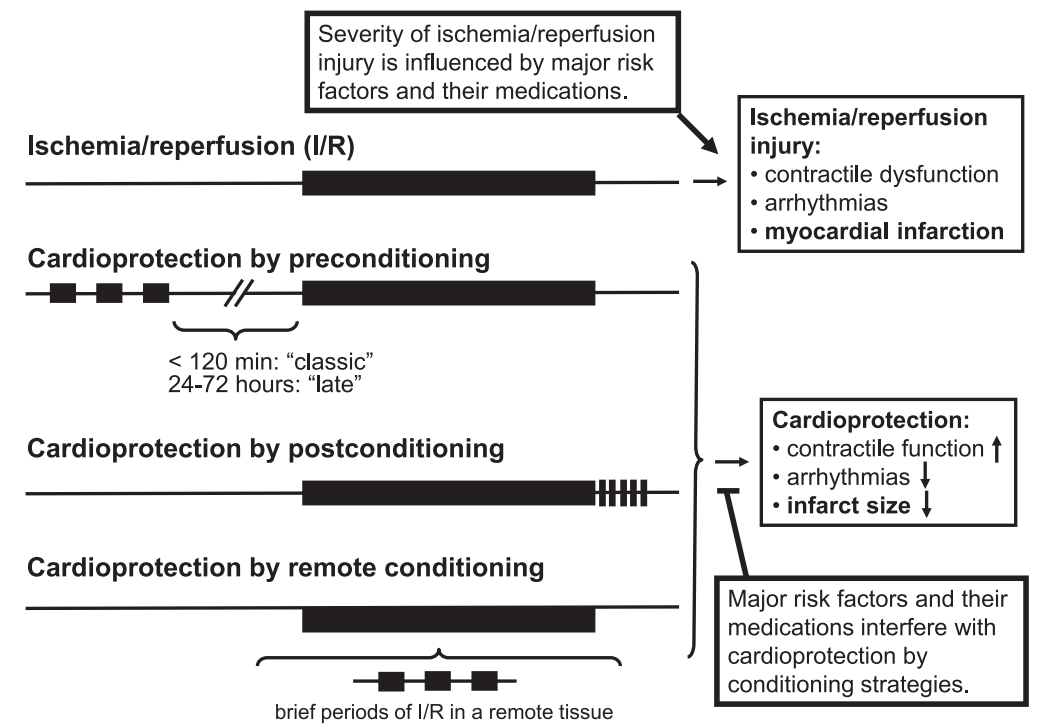

Fig. 1. The concept of ischemia/reperfusion injury and cardioprotection by pre-, post-, and remote conditioning is expressed graphically in the figure, where black bars denote periods of ischemia. Myocardial ischemia and reperfusion lead to "ischemia/reperfusion injury" characterized by the development of contractile dysfunction, arrhythmias, and tissue necrosis (infarction). Ischemic preconditioning is a well described acute and subacute adaptive response in which brief exposure to ischemia/reperfusion markedly enhances the ability of the heart to withstand a subsequent ischemia/ reperfusion injury. In this diagram, three brief periods of ischemia are used to precondition the myocardium against a subsequent period of "test" ischemia that is longer than the preconditioning periods. Preconditioning induces protection in a biphasic pattern. Brief cycles of ischemia/reperfusion applied after a longer period of ischemia also confer cardioprotection against the consequences of myocardial ischemia/reperfusion, a phenomenon called ischemic postconditioning. Brief cycles of ischemia/reperfusion applied in a remote cardiac tissue or remote organ, e.g., kidney, limbs, before, during, or right after a longer period of cardiac ischemia also provide cardioprotection, a phenomenon called remote conditioning. The cardioprotective effect of conditioning strategies results in attenuation of ischemia/reperfusion injury characterized by improvement of postischemic contractile function, decrease in the occurrence and severity of arrhythmias, and reduction of infarct size. Major cardiovascular risk factors and their medications influence the severity of ischemia/reperfusion injury and interfere with the cardioprotective effect of conditioning.

cellular signaling mechanisms, thereby possessing a "hidden cardiotoxicity" that may manifest latently in the ischemic heart as increased sensitivity to ischemic challenge or a decreased capability to adapt to an ischemic challenge, i.e., attenuated cardioprotection achieved by conditioning.

\section{Experimental Approaches to Cardioprotection}

\section{A. Cardioprotection through Preconditioning}

Cardioprotection elicited by ischemic preconditioning remains one of the most powerful therapeutic interventions for limiting infarct size after acute ischemia/reperfusion injury. Despite ongoing intensive investigation, the actual mechanisms underlying its cardioprotective effect and their interaction remain largely unclear. A large number of signaling pathways are recruited at the cardiomyocyte sarcolemma through the activation of cell surface receptors by their endogenous ligands. Many of these signal transduction pathways appear to terminate at the mitochondria, and it is in this area where most of the recent research has been focused (Fig. 2). A comprehensive review of all of the investigated mechanisms is beyond the scope of this review. The interested reader is referred to comprehensive reviews published elsewhere on the topics of ischemic preconditioning and its signal transduction (Heusch et al., 2008; Hausenloy, 2013).
1. Mitochondria and Preconditioning. Mitochondria appear to play two critical roles in the setting of ischemic preconditioning. Before the index ischemic event and in response to the preconditioning stimulus, mitochondria are known to release signaling reactive oxygen species (ROS) that then activate key mediators of cardioprotection, which subsequently prevent the opening of the mitochondrial permeability transition pore (MPTP) in the first few minutes of myocardial reperfusion, thereby attenuating myocardial reperfusion injury and limiting infarct size.

a. The mitochondrial permeability transition pore and preconditioning. The mechanism through which the signaling ROS are generated in response to the ischemic preconditioning stimulus is not clear, but one suggestion has implicated the activation of the mitochondrial ATP-sensitive potassium channel ( $\left.\mathrm{K}_{\mathrm{ATP}}\right)$, which is related to mitochondrial connexin $43(\mathrm{Cx} 43)$ (Heinzel et al., 2005) and appears to be mediated via protein kinase $\mathrm{G}$ (PKG) and mitochondrial protein kinase $\mathrm{C}(\mathrm{PKC}) \varepsilon$ (Costa and Garlid, 2008). The $\mathrm{K}^{+}$ influx into mitochondria is believed to induce matrix alkalinization that then results in the production of superoxide from complex I of the electron transport chain (Soetkamp et al., 2014).

A number of experimental studies have linked ischemic preconditioning-induced cardioprotection to the inhibition of MPTP opening at the onset of reperfusion. 


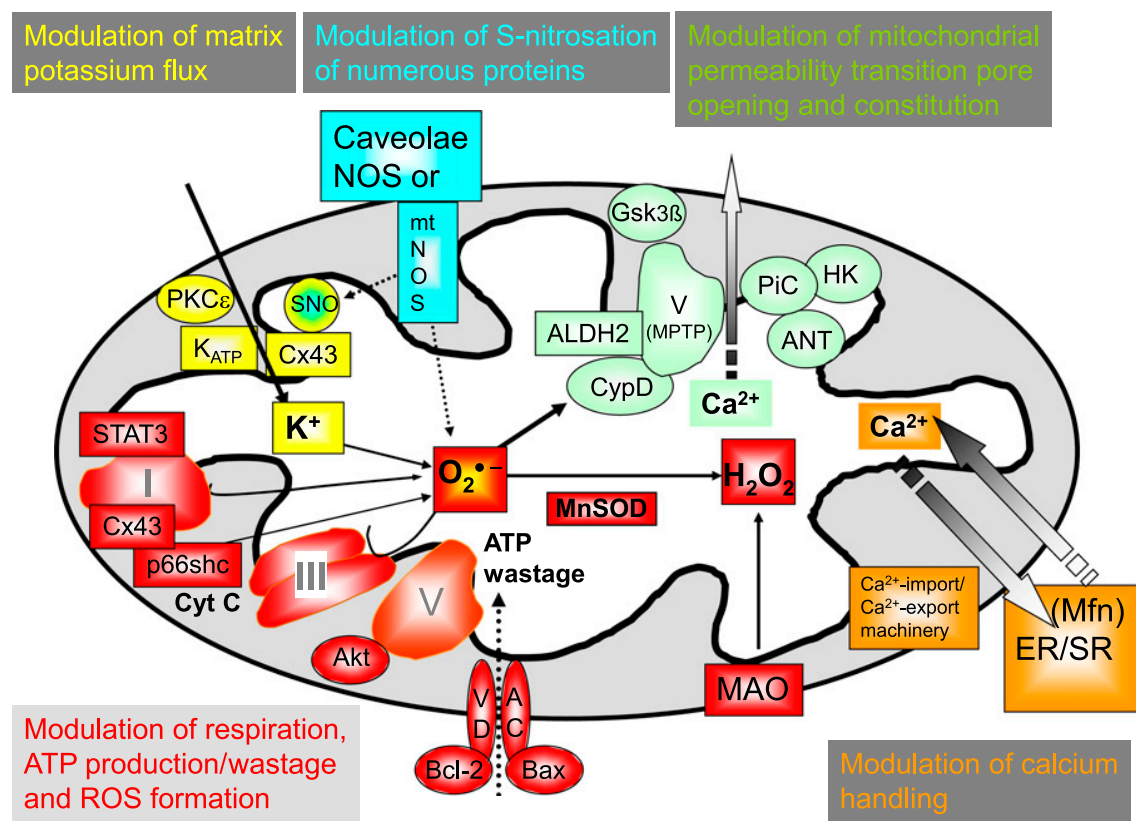

Fig. 2. Mitochondrial reactive oxygen species (ROS, including $\mathrm{O}_{2}^{-}, \mathrm{H}_{2} \mathrm{O}_{2}$ ) are at the center of cardioprotection and/or irreversible injury depending on the timing and quantity of their generation. Several mitochondrial proteins contribute to the generation of ROS through modulation of proteins of the respiratory chain (Cx43, STAT3, p66shc) or directly [monoamine oxidases (MAO)]. High amounts of ROS at the time of reperfusion contribute to irreversible tissue injury, probably by facilitating opening of the mitochondrial permeability transition pore (MPTP). The proteins contributing to the formation of MPTP are still under investigation, but dimerization of complex V or protein complexes involving adenine-nucleotide transporter (ANT), hexokinase (HK), and the phosphate carrier (PiC) has been proposed. Many factors apart from ROS are important for MPTP opening, including binding of cyclophilin-D (CypD), calcium $\left(\mathrm{Ca}^{2+}\right)$, and ADP. Mitochondrial $\mathrm{Ca}^{2+}$ concentration and homeostasis is influenced by the close interaction with the SR/ER and specialized proteins for such interaction like mitofusins (Mfn). Under pathophysiological conditions, ADP can be generated by the reversed mode of complex V using ATP as substrate to maintain the inner mitochondrial membrane proton gradient. ATP will pass the outer mitochondrial membrane through the voltage-gated anion channel (VDAC) and proteins, such as Bcl2 or Bax, affecting channel open probability. Some other proteins modifying MPTP opening have been described, such as GSK-3 $\beta$, aldehyde dehydrogenase 2 (ALDH2), and PKC $\varepsilon$. Although high concentrations of ROS are detrimental, low amounts of ROS can trigger a cardioprotective state and are central to the endogenous protection by pre- and postconditioning. In this context, increases in mitochondrial potassium $\left(\mathrm{K}^{+}\right)$lead to increased ROS formation and are central to endogenous cardioprotection. Here, mitochondrial $\mathrm{K}_{\mathrm{ATP}}$ but also $\mathrm{Cx} 43$ play important roles. Nitrosylation of thiol groups (SNO) are also important for protein activity, and nitric oxide can be derived either by a mitochondrial nitric oxide synthase (mtNOS) or by a NOS isoform in close proximity to mitochondria, transported by caveolae-like structures into the mitochondria.

The precise mechanism through which this is achieved remains undetermined but may involve the following: 1) activation of prosurvival pathways, such as the reperfusion injury salvage kinase (RISK) or survivor activating factor enhancement (SAFE) signaling pathways, that then act to prevent MPTP opening either in a direct or indirect manner (Hausenloy et al., 2009, 2011). There is evidence to suggest that one particular downstream mediator, glycogen synthase kinase- $3 \beta$ (GSK-3 $\beta$ ), appears to mediate cardioprotection through the inhibition of the MPTP, although the mechanism through which this is achieved is unclear (Juhaszova et al., 2004); 2) activation of the mitochondrial $\mathrm{K}_{\mathrm{ATP}}$ channel, which via mitochondrial $\mathrm{PKC} \varepsilon$, results in ROS-mediated inhibition of MPTP opening (Costa and Garlid, 2008); and 3) attenuation of oxidative stress generated during myocardial ischemia, thereby preventing MPTP opening at reperfusion (Clarke et al., 2008).

Since 2007, several key developments have arisen with respect to the MPTP and its role in acute ischemia/reperfusion injury. Although mitochondrial cyclophilin D has been established as a regulator of the
MPTP, the precise identities of the components of the MPTP remain unknown. Recent experimental studies have suggested that dimers of mitochondrial ATP synthase may constitute the MPTP (Bonora et al., 2013; Giorgio et al., 2013). Interestingly, an insight into the potential physiological role of the MPTP was provided by Elrod et al. (2010) who reported that mice deficient in mitochondrial cyclophilin D were more susceptible to calcium overload, suggesting that the MPTP may mediate mitochondrial calcium efflux, a mechanism that had been first proposed in 1992 (Altschuld et al., 1992). Another important discovery was the identity of the mitochondrial calcium uniporter (Baughman et al., 2011; De Stefani et al., 2011) and the surprising observation that mice deficient in the uniporter were not protected from myocardial infarction despite being resistant to MPTP opening (Pan et al., 2013).

b. Mitochondrial connexin-43 and preconditioning. Recent experimental data have suggested that the gap junction sarcolemmal protein, $\mathrm{Cx} 43$, is also present in cardiac subsarcolemmal inner mitochondrial membranes, where it acts as a signaling mediator of ischemic preconditioning but not postconditioning (reviewed 
in Schulz et al., 2007). Cx43 is believed to form hemichannels in the inner mitochondrial membrane, thereby facilitating complex I function and the influx of $\mathrm{K}^{+}$into mitochondria in response to the ischemic preconditioning stimulus (Boengler et al., 2012, 2013a; Soetkamp et al., 2014). The activation of the RISK or SAFE pathways is not involved in the protective function of $\mathrm{Cx} 43$ in ischemic preconditioning (Sanchez et al., 2013).

c. Mitochondria and new forms of cell death. The majority of experimental studies investigating the beneficial effects of conditioning the heart have focused on preventing cardiomyocyte death due to necrosis and/or apoptosis, and in this regard the mitochondria play a pivotal role. More recently, two further forms of cell death have been described. Both autophagy (including mitophagy) and regulated cell necrosis appear to be relevant to cardiomyocyte death induced by acute ischemia/reperfusion injury. Only an overview can be provided here, and the interested reader is referred to more comprehensive review articles (Giricz et al., 2012; Kaczmarek et al., 2013).

Autophagy is an evolutionarily conserved process that mediates the degradation of cytoplasmic components via the lysosomal pathway under conditions of cellular stress. It has been suggested that autophagy may be an adaptive response to protect the cell against myocardial ischemia. Autophagy can be activated in response to ischemic preconditioning, whereas its activation at the time of reperfusion is thought to be deleterious (reviewed in Giricz et al., 2012). Mitophagy allows the removal of defective mitochondria and may also provide a cardioprotective response (Kubli and Gustafsson, 2012). This process is initiated by mitochondrial fragmentation and mitochondrial membrane depolarization that induces the translocation of the cytosolic ubiquitin ligase, parkin, to the mitochondrial outer membrane where it binds to mitofusin 2 , which in itself has to be phosphorylated by phosphatase and tensin homologue (PTEN)-induced kinase 1 (Chen and Dorn, 2013), resulting in the removal of the damaged mitochondria. Abolishing mitophagy by knockout of parkin abolishes cardioprotection by ischemic preconditioning (Huang et al., 2011).

Necrosis was previously considered to be an accidental, unregulated form of cell death. However, there also appears to be a regulated form of necrotic cell death, termed "necroptosis" or "programmed necrosis" (reviewed by Kaczmarek et al., 2013). It is initiated by tumor necrosis factor $\alpha(\mathrm{TNF} \alpha)$ death domain receptor activation, the receptor-interacting protein 1 and 3 kinases, the mixed lineage kinase domain-like protein, and mitochondrial phosphoglycerate mutase/protein phosphatase, which then activates dynamin-related protein 1 (Drp1)mediated mitochondrial fission resulting in cell death (Wang et al., 2012). Importantly, pharmacologic inhibition of this novel death pathway has been reported to limit infarct size and prevent adverse post-MI left ventricular remodeling (Lim et al., 2007; Oerlemans et al., 2012).

d. Mitochondrial dynamics and cardioprotection. Mitochondria are no longer considered to be static organelles but are dynamic structures that are able to change their morphology by undergoing either fusion to generate elongated mitochondria, which allows replenishment of damaged mitochondrial DNA, or fission to produce fragmented mitochondria to replace damaged mitochondria by mitophagy (reviewed in Ong and Hausenloy, 2010; Ong et al., 2013). Interestingly, cardiac mitochondria have been demonstrated to undergo fragmentation during myocardial ischemia under the control of the mitochondrial fission protein Drp1 (Ong et al., 2010). Pharmacologic or genetic inhibition of Drp1mediated mitochondrial fission induced by ischemia has been reported to prevent MPTP opening and reduce infarct size (Ong et al., 2010; Wang et al., 2011a; Disatnik et al., 2013). Somewhat surprisingly, the ablation of cardiac mitofusin 1 and 2 (known mitochondrial fusion proteins) also prevented MPTP opening and rendered hearts resistant to acute ischemia/reperfusion injury (Papanicolaou et al., 2011, 2012). This unexpected result may be due to the pleiotropic nonfusion effects of these mitochondrial fusion proteins that include apoptosis induction, mediation of mitophagy, and tethering the sarcoplasmic reticulum to the mitochondria (de Brito and Scorrano, 2008a,b; Wang et al., 2012). A recent study has shown that pharmacologic preconditioning using nitrite protected a cardiac cell line by inhibiting ischemia-induced mitochondrial fission through the activation of protein kinase A (Pride et al., 2014). Whether ischemic preconditioning and postconditioning exert their cardioprotective effect by modulating mitochondrial morphology is not known.

\section{B. Cardioprotection through Postconditioning}

One major limitation of ischemic preconditioning has been the necessity to apply the therapeutic intervention before the sustained index myocardial ischemia, the onset of which is unpredictable in patients presenting with MI. The discovery in 2003 of ischemic postconditioning by interrupting myocardial reperfusion with several cycles of short-lived ischemia has overcome this limitation (Zhao et al., 2003). The clinical applicability of ischemic postconditioning was realized only 2 years later in ST-segment elevated MI (STEMI) patients treated by percutaneous coronary intervention (PCI) using reinflation of the coronary angioplasty balloon to interrupt myocardial reperfusion (Staat et al., 2005) (see section III for further clinical application of ischemic postconditioning). The protection afforded by ischemic postconditioning has been reproduced in most species tested, although suitable algorithms may be model- and species-dependent (Skyschally et al., 2009). The modification of the reperfusion phase had been reported 
previously to confer cardioprotection by more gentle reperfusion (Musiolik et al., 2010).

In terms of the mechanistic pathway underlying ischemic postconditioning, many of the signaling pathways, but not all (Heusch et al., 2006), are shared with ischemic preconditioning. Briefly, autacoids activate prosurvival signal transduction pathways, the majority of which converge on mitochondria and prevent MPTP opening at the time of reperfusion. A comprehensive review of all of the investigated mechanisms is beyond the scope of this review and we will only focus on the major developments since 2007. The interested reader is referred to comprehensive reviews published elsewhere on the topics of ischemic postconditioning (Burley and Baxter, 2009; Ovize et al., 2010; Shi and Vinten-Johansen, 2012; Hausenloy, 2013).

1. Autacoid Mediators of Postconditioning. Initial experimental studies using pharmacologic antagonists had implicated adenosine to be a key mediator of postconditioning through activation of the adenosine $\mathrm{A}_{2 \mathrm{~A}}$ receptor (Kin et al., 2005), $\mathrm{A}_{2 \mathrm{~B}}$ receptor (Philipp et al., 2006), or $\mathrm{A}_{3}$ receptor (Kin et al., 2005; Philipp et al., 2006), but not the $\mathrm{A}_{1}$ receptor (Kin et al., 2005; Donato et al., 2007; Xi et al., 2008). A subsequent study found that mice deficient for the myocardial adenosine $\mathrm{A}_{2 \mathrm{~A}}$ receptor were resistant to ischemic postconditioning (Morrison et al., 2007). Since 2007, an increasing number of autacoid mediators of postconditioning have been described, including bradykinin (Penna et al., 2007; Xi et al., 2008), opioids (Jang et al., 2008; Pateliya et al., 2008; Zatta et al., 2008), TNF $\alpha$ (Lacerda et al., 2009), and sphingosine (Jin et al., 2008; Vessey et al., 2008a,b).

2. Delaying the Correction of $p H$ at Reperfusion. The acidic intracellular conditions produced during myocardial ischemia exert a strong inhibitory effect on the MPTP, keeping it closed during ischemia, despite calcium overload, increased inorganic phosphate, oxidative stress, and ATP depletion. In the first few minutes of reperfusion, the washout of myocardial lactate and activation of the $\mathrm{Na}^{+}-\mathrm{H}^{+}$exchanger and $\mathrm{Na}^{+}-\mathrm{HCO}_{3}{ }^{-}$ cotransporter rapidly correct the intracellular acidosis, thereby releasing the inhibition on the MPTP and allowing the latter to open at the time of reperfusion (Halestrap et al., 2004; Yellon and Hausenloy, 2007). A number of experimental studies reported that ischemic postconditioning may prevent MPTP opening by delaying the restoration of physiologic $\mathrm{pH}$ at the onset of reperfusion (Cohen et al., 2007; Fujita et al., 2007), although the actual mechanism through which this might be achieved is not clear. Whether the stuttering reperfusion of the postconditioning protocol inhibits MPTP opening by delaying the washout of the myocardial lactate, attenuating oxidative stress production, or activating the RISK or SAFE pathway is unclear.

3. Mitochondria and Postconditioning. Experimental studies suggest that ischemic postconditioning prevents myocardial reperfusion injury and limits infarct size by inhibiting MPTP opening (Argaud et al., 2005). As with ischemic preconditioning, the mechanism through which this is achieved is not clear, but a number of potential signaling pathways have been proposed: 1) the activation of the prosurvival cardioprotective pathways, such as the RISK, SAFE, and NO-cGMP-PKG pathways, at the onset of reperfusion inhibit MPTP opening (Hausenloy et al., 2005, 2011; Bopassa et al., 2006; Heusch et al., 2008, 2011; Boengler et al., 2011a; Andreadou et al., 2014); 2) the delayed restoration in intracellular $\mathrm{pH}$ may inhibit MPTP opening (Cohen et al., 2007); and 3) the reduction in ROS generated at reperfusion may prevent MPTP opening (Clarke et al., 2008).

\section{Cardioprotection through Pharmacologic Conditioning}

Elucidation of the signaling pathways underlying ischemic conditioning in the heart has helped to identify a number of novel therapeutic targets for cardioprotection. These include targets in the signal transduction pathways linking the cell membrane to the mitochondria and direct targets in the mitochondria. A number of pharmacologic agents capable of mimicking the cardioprotective effects of ischemic conditioning continue to be investigated in the experimental setting, but there appear to be species differences, e.g., cyclosporine-A does not protect the rat heart (De Paulis et al., 2013). Some of these agents have been investigated in the clinical setting already. The most promising pharmacologic cardioprotective agents and their potential targets include: cyclosporine-A (MPTP inhibition); metoprolol, matrix metalloproteinase (MMP) inhibition, glucagon-like peptide 1 (GLP-1) analogs (RISK pathway); and nitrite/nitrates and soluble guanylate cyclase activators (NO-cGMP-PKG pathway) (reviewed in Evgenov et al., 2006; Stasch et al., 2011; Sharma et al., 2012; Andreadou et al., 2014; Bice et al., 2014; Rassaf et al., 2014).

\section{Cardioprotection through Remote Conditioning}

The major disadvantage of ischemic preconditioning and postconditioning as therapeutic interventions for limiting acute myocardial ischemia/reperfusion injury is that they both require the intervention to be applied directly to the heart, thereby limiting their clinical applicability. In this regard, the discovery in 1993 (Przyklenk et al., 1993) that the cardioprotective stimulus could be applied to remote myocardium and later to a remote organ away from the heart, was a major advance. This phenomenon has been termed "remote ischemic conditioning" (reviewed in Hausenloy and Yellon, 2008; Vinten-Johansen and Shi, 2013). However, the major breakthrough that facilitated the translation of remote ischemic conditioning into the clinical setting was the discovery in the experimental setting that the cardioprotective stimulus could be applied to the musculoskeletal tissue of the hindlimb (Birnbaum et al., 1997; 
Oxman et al., 1997). This was followed by the discovery in human volunteers that the cardioprotective stimulus could be applied to the arm or leg in a noninvasive manner by simply inflating and deflating a blood pressure cuff or similar device (Günaydin et al., 2000; Kharbanda et al., 2002).

An additional advantage with remote ischemic conditioning is its ability to confer cardioprotection when initiated at different time points in relation to acute ischemia/reperfusion injury. It can be applied before myocardial ischemia (remote ischemic preconditioning) (Przyklenk et al., 1993); after the onset of myocardial ischemia but before reperfusion (remote ischemic perconditioning) (Schmidt et al., 2007); at the onset of myocardial reperfusion (remote ischemic postconditioning) (Andreka et al., 2007); and even after 15 minutes of reperfusion has elapsed (remote ischemic delayed postconditioning) (Basalay et al., 2012). Remote conditioning interventions thereby lend themselves to application in a number of different clinical settings of acute ischemia/reperfusion injury (see section III). Moreover, repeated daily episodes of remote ischemic postconditioning over a period of 28 days after MI in a rat model of acute ischemia/reperfusion injury have been reported to have beneficial effects on post-MI remodeling (Wei et al., 2011).

Despite its discovery in 1993, the actual mechanism underlying the cardioprotective effect of remote conditioning remains unclear. The signal transduction pathway can be divided into three stages: 1) the application of the "conditioning" stimulus to the remote organ or tissue results in the generation of a cardioprotective signal, the nature of which is unclear; 2 ) the mechanism through which the cardioprotective signal is conveyed to the heart is currently unclear but is believed to involve both neural and circulating humoral components; and 3) the recruitment of established cardioprotective signaling pathways within the cardiomyocyte (reviewed in Hausenloy and Yellon, 2008). Dissection of the individual contributions of these three sequential signaling steps has been an experimental challenge that remains unsolved. The current paradigm suggests that the conditioning stimulus within the remote organ or tissue generates autacoids, such as adenosine, bradykinin, and opioids, which result in the stimulation of the neural pathway to that remote organ or tissue (Liem et al., 2002; Jensen et al., 2012; Redington et al., 2012). The neural pathway then relays the cardioprotective signal to the brain stem nuclei (Lonborg et al., 2012), where a humoral factor(s), as yet unidentified, is released into the circulation and carried to the heart to mediate the cardioprotective effect. Recently, involvement of the SDF-1 $\alpha /$ CXCR 4 axis has been shown (Davidson et al., 2013). Also, cardioprotection by remote ischemic preconditioning of the rat heart was recently shown to be mediated by extracellular vesicles released by brief periods of ischemia and acting as potential carriers of cardioprotective substances (Giricz et al., 2014). A comprehensive discussion of the potential mechanisms underlying remote ischemic conditioning is beyond the scope of this review, and the reader is referred to comprehensive reviews on the subject (Hausenloy and Yellon, 2008; Vinten-Johansen and Shi, 2013). The clinical application of this phenomenon is dealt with in the next section.

\section{Clinical Approaches to Cardioprotection}

There are now a number of studies that have examined cardioprotection by ischemic preconditioning, ischemic postconditioning, and remote conditioning in various clinical scenarios (Heusch, 2013).

\section{A. Ischemic Preconditioning}

Conceptually, ischemic preconditioning has been associated with preinfarction angina, i.e., unstable angina preceding acute MI. It is known that preinfarction angina is associated with better clinical outcome than an abrupt acute MI without preceding episodes of angina (Heusch, 2001; Rezkalla and Kloner, 2004). The causal attribution of protection with preinfarction angina to ischemic preconditioning rather than collateral recruitment or more rapid reperfusion, as well as to the early versus the delayed form of ischemic preconditioning, remains unclear. Although conceptually inferred for preinfarction angina, ischemic preconditioning has been more empirically studied in interventional and surgical revascularization protocols (Fig. 3).

During repeated balloon angioplasty, ECG alterations, pain sensation, lactate production, and creatine kinase release were found to be attenuated during the second compared with the first coronary occlusion period, and this was taken as evidence of ischemic preconditioning (Heusch, 2001). With the use of pharmacologic antagonists, the causal involvement of adenosine, opioids, $\alpha$-adrenoceptor activation, and $\mathrm{K}_{\mathrm{ATP}}$ was demonstrated. However, a caveat must be noted because reduced ST-segment elevation can be dissociated from reduced infarct size (Birincioglu et al., 1999) such that the selected endpoint of ischemic preconditioning's protection may be critical for successful clinical translation. There are also a number of studies where an ischemic preconditioning algorithm was used in coronary artery bypass graft or valvular surgery, and protection was seen in terms of reduced release of serum biomarkers (creatine kinase-MB, troponin I or T) (Jenkins et al., 1997; Lu et al., 1997; Szmagala et al., 1998; Li et al., 1999; Teoh et al., 2002a,b; Buyukates et al., 2005; Codispoti et al., 2006; Ji et al., 2007; Amr and Yassin, 2010). However, not all studies were positive (Alkhulaifi et al., 1994; Perrault et al., 1996; Cremer et al., 1997; Kaukoranta et al., 1997; Illes and Swoyer, 1998; PêgoFernandes et al., 2000; Wu et al., 2001; Ghosh and Galinanes, 2003; Jebeli et al., 2010). Both positive and 


\section{Ischemic preconditioning}

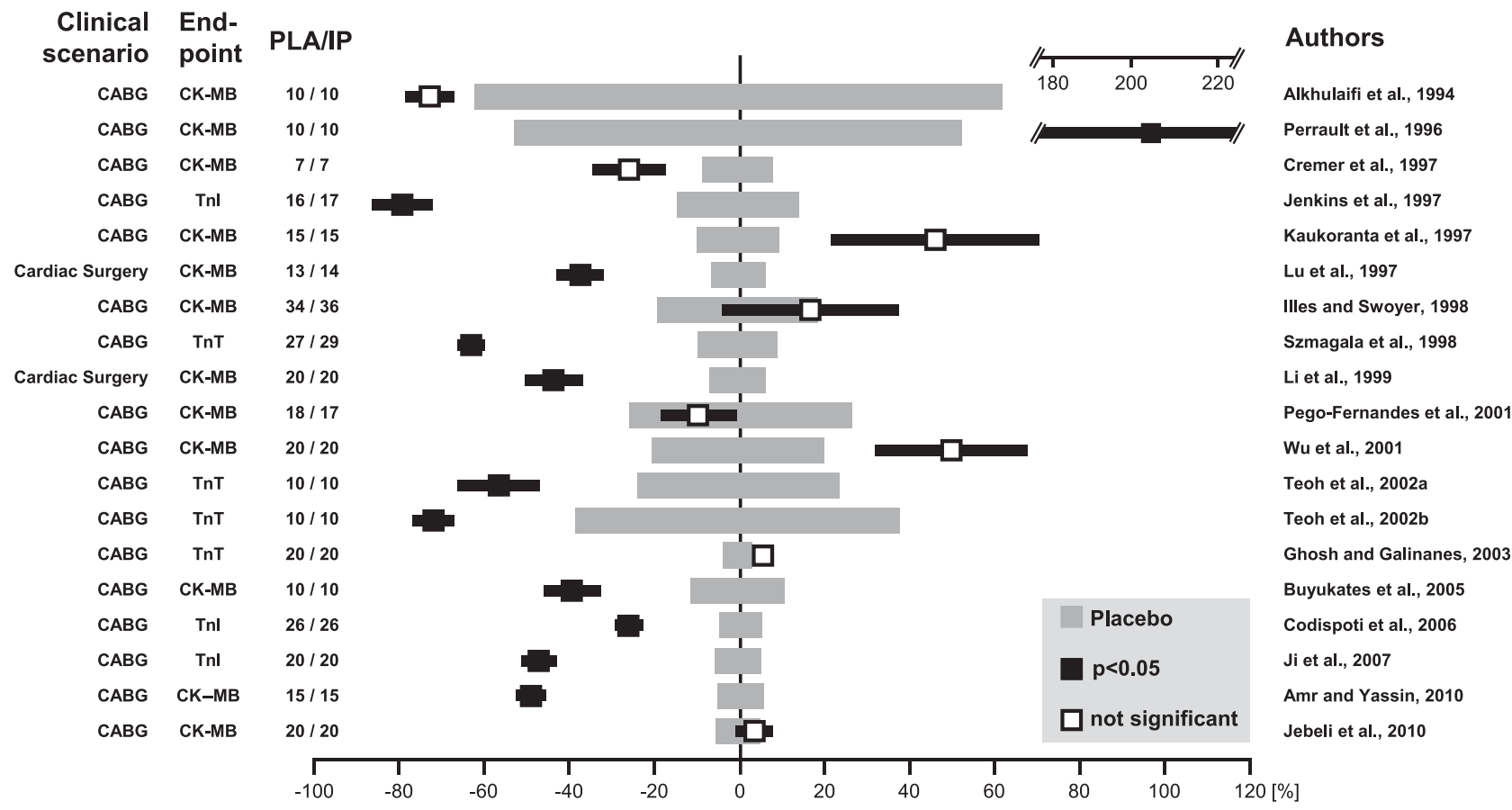

Fig. 3. Forest plot on the available clinical studies (state December 2013) on ischemic preconditioning. Gray bars indicate the standard error of the mean in the placebo group, black bars the \% infarct size reduction with its standard error in the conditioned group (updated from Heusch, 2013). CABG, coronary artery bypass grafting; CK-MB, creatine kinase-MB; IP, ischemic preconditioning; PLA, placebo; TnI, troponin I; TnT, troponin T.

negative studies suffer from small cohort sizes and lack of clinical outcome as endpoint. Nevertheless, the impression is that ischemic preconditioning can be used to induce protection in elective cardiac surgery, and a metaanalysis of published studies suggests clinical benefit in terms of reduced arrhythmias, less inotrope support requirement, and reduced intensive care unit stay (Walsh et al., 2008).

\section{B. Ischemic Postconditioning}

Ischemic postconditioning has been used in patients undergoing primary PCI for an acute MI (Fig. 4). The landmark study by Staat et al. (2005) appeared only 2 years after the original experimental report of ischemic postconditioning in dogs (Zhao et al., 2003). Several studies demonstrated reduced infarct size by reduced biomarker release (creatine kinase, creatine kinase$\mathrm{MB}$, troponin I) or by gadolinium-contrast magnetic resonance imaging ( $\mathrm{Ma}$ et al., 2006; Luo et al., 2007; Yang et al., 2007; Laskey et al., 2008; Luo et al., 2008a,b; Thibault et al., 2008; Li et al., 2009; Zhao et al., 2009b; Lonborg et al., 2010; Xue et al., 2010; Garcia et al., 2011; Ji et al., 2011; Liu et al., 2011a; Luo et al., 2011; Durdu et al., 2012; Thuny et al., 2012; Liu et al., 2013; Mewton et al., 2013). However, not all studies have reported positive findings (Sorensson et al., 2010; Freixa et al., 2012; Tarantini et al., 2012; Ugata et al., 2012; Dwyer et al., 2013; Elzbieciak et al., 2013; Hahn et al., 2013). The sample size of the study cohorts was small, making them sensitive to false-negative type
II errors. A systematic underestimation of the protective potential of ischemic postconditioning may result from lack of direct stenting. Direct stenting removes any residual stenosis and prevents coronary microembolization from the culprit lesion (Loubeyre et al., 2002) when further manipulated by the postconditioning maneuver (Heusch, 2012). With use of direct stenting, the consequences of immediate full reperfusion are compared with those of a postconditioning algorithm, without any interference by a residual stenosis or by coronary microembolization. Also, Ovize and colleagues who consistently reported protection with ischemic postconditioning always inflated the balloon upstream of the stent (Staat et al., 2005; Thibault et al., 2008; Thuny et al., 2012). A larger clinical trial recently failed to observe reduced peak creatine kinaseMB or a significant benefit in clinical outcome from ischemic postconditioning, but unfortunately this trial did not use direct stenting in most patients (Hahn et al., 2013).

\section{Remote Ischemic Conditioning}

In recent years, remote ischemic conditioning has become the most popular form of mechanical cardioprotection, because the procedure is noninvasive, predictable, precise, safe, and notably avoids manipulation of the coronary culprit lesion (Fig. 5). Remote ischemic preconditioning has been used in elective interventional revascularization (Iliodromitis et al., 2006; Hoole et al., 2009; Ahmed et al., 2013; Luo et al., 2013; Prasad et al., 
Ischemic postconditioning

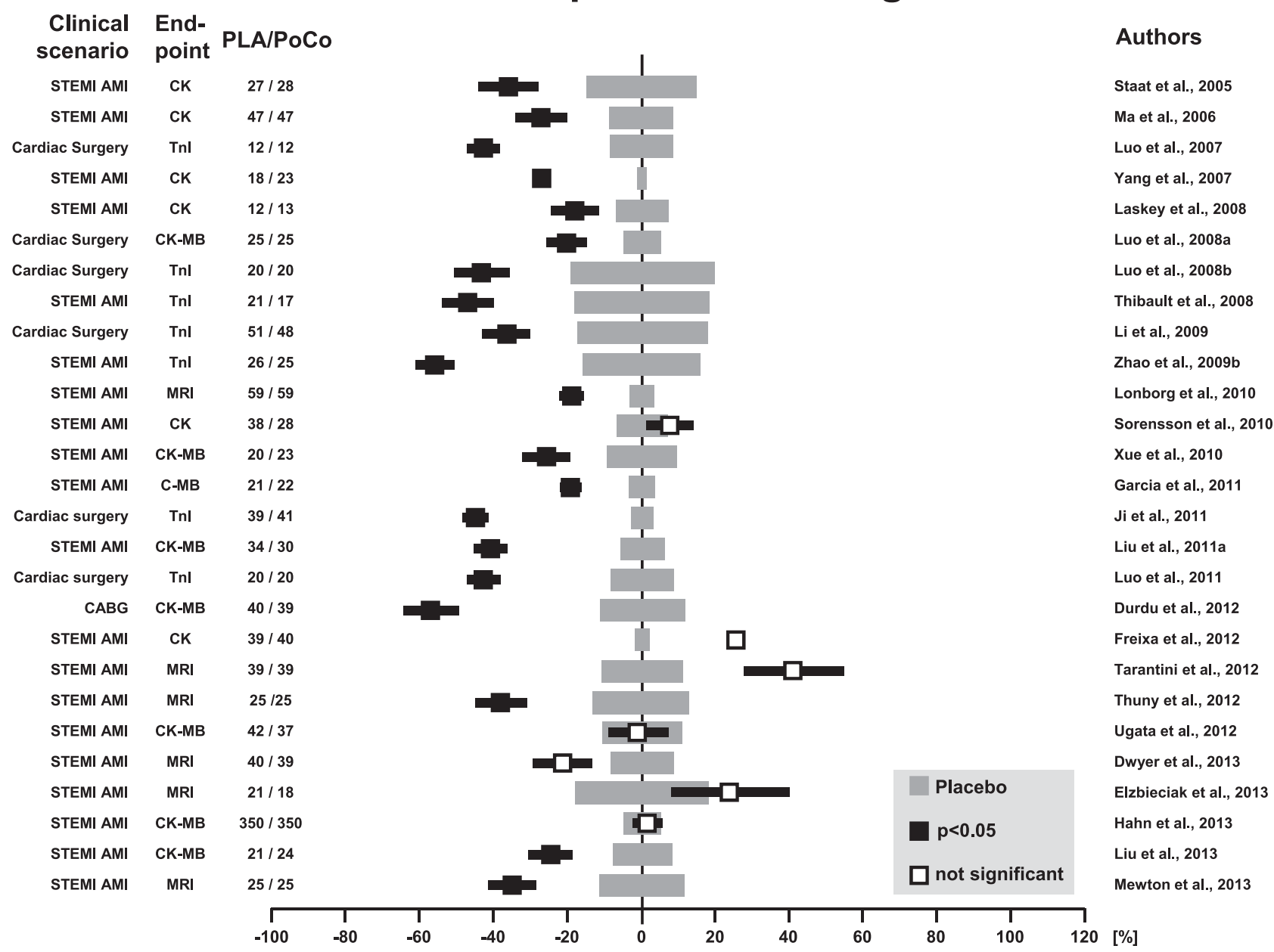

Fig. 4. Forest plot on the available clinical studies (state December 2013) on ischemic postconditioning. Gray bars indicate the standard error of the mean in the placebo group, black bars the \% infarct size reduction with its standard error in the conditioned group (updated from Heusch, 2013). AMI, acute myocardial infarction; CABG, coronary artery bypass grafting; CK, creatine kinase; CK-MB, creatine kinase-MB; MRI, magnetic resonance imaging; TnI, troponin I.

2013) and in surgical coronary revascularization (Günaydin et al., 2000; Hausenloy et al., 2007; Venugopal et al., 2009; Ali et al., 2010; Hong et al., 2010, 2012; Rahman et al., 2010; Thielmann et al., 2010, 2013; Wagner et al., 2010; Karuppasamy et al., 2011; Heusch et al., 2012b; Kottenberg et al., 2012, 2014a; Lomivorotov et al., 2012; Lucchinetti et al., 2012; Young et al., 2012; Saxena et al., 2013). The procedure has also been applied in other forms of cardiac surgery (Cheung et al., 2006; Li et al., 2010; Zhou et al., 2010; Choi et al., 2011; Luo et al., 2011; Wu et al., 2011a; Lee et al., 2012; Pavione et al., 2012; Xie et al., 2012; Young et al., 2012; Albrecht et al., 2013; Jones et al., 2013; Meybohm et al., 2013; Pepe et al., 2013). Not all studies reported infarct size reduction, using biomarker release or imaging as endpoints. A common feature of all the negative studies appears to be the use of propofol anesthesia in some form; propofol has been demonstrated to abrogate the protection by remote ischemic preconditioning (Kottenberg et al., 2012, 2014a; Bautin et al., 2013).
A few studies have also used a remote conditioning procedure during an ongoing acute $\mathrm{MI}$ before primary PCI; increased myocardial salvage was seen in one study (Botker et al., 2010), but no significant reduction in infarct size by biomarker release or imaging (Botker et al., 2010; Munk et al., 2010; Rentoukas et al., 2010). One recent study demonstrated reduced infarct size, as assessed by biomarker release and magnetic resonance imaging, when the remote lower limb conditioning protocol was started in a postconditioning mode at the onset of reperfusion in patients with acute MI (Crimi et al., 2013). Three further studies even reported reduced all-cause mortality (secondary endpoint) in patients undergoing a remote conditioning protocol before elective PCI (Davies et al., 2013), emergency PCI (Sloth et al., 2014), or surgical coronary revascularization (Thielmann et al., 2013). Another recent study reported no clinical benefit in patients undergoing elective cardiac surgery with a combined remote ischemic pre- and postconditioning protocol; however, 


\section{Remote ischemic conditioning}

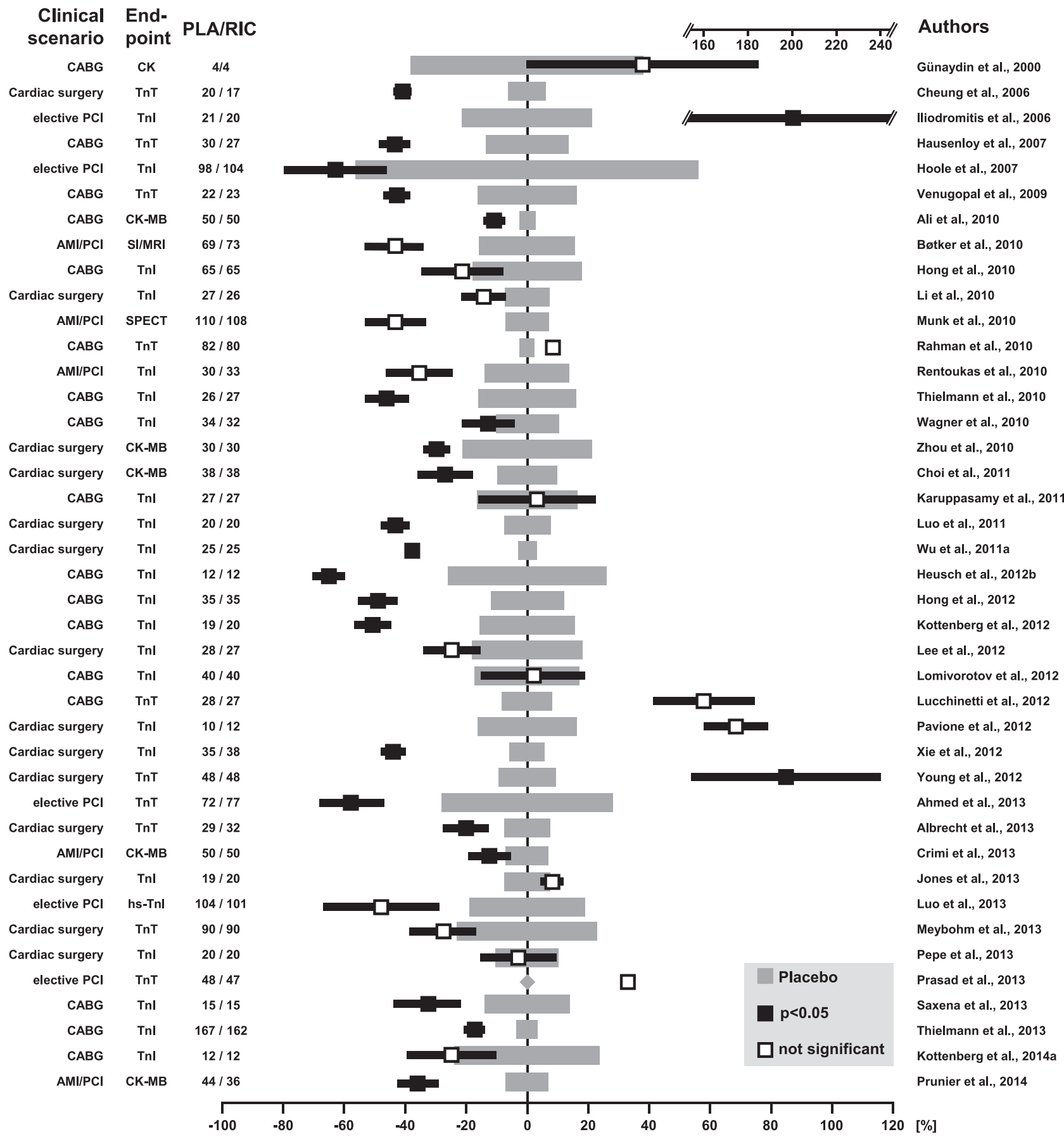

Fig. 5. Forest plot on the available clinical studies (state December 2013) on remote ischemic conditioning. Gray bars indicate the standard error of the mean in the placebo group, black bars the \% infarct size reduction with its standard error in the conditioned group (updated from Heusch, 2013). AMI, acute myocardial infarction; CABG, coronary artery bypass grafting; CK, creatine kinase; CK-MB, creatine kinase-MB; MRI, magnetic resonance imaging; SI, salvage index; TnI, troponin I; TnT, troponin T.

this study also used propofol and did not report protection in terms of biomarker release or imaging endpoints (Hong et al., 2014). Also, no additive protection of local ischemic postconditioning with remote ischemic preconditioning was seen in the small-scale RIPOST-MI study in patients undergoing primary PCI for acute MI (Prunier at al., 2014). We therefore await the results of several ongoing multicenter trials on remote conditioning where mortality is a primary endpoint; these include ERICCA (Hausenloy et al., 2012) (NCT 1247545), RIPHeart (NCT 01067703), or CONDI II (NCT 01857414).

\section{Pharmacologic Postconditioning}

Our increasing understanding of the mechanisms underlying ischemic postconditioning has identified 
a vast array of signaling mediators, which can be targeted by pharmacologic agents to recapitulate the cardioprotective effects of ischemic postconditioning. In this regard, a number of pharmacologic approaches to limit infarct size in STEMI patients undergoing primary PCI have been investigated (reviewed in Sharma et al., 2012; Hausenloy et al., 2013a). Unfortunately, many of these studies have failed to demonstrate any cardioprotective effect in the clinical setting, despite promising experimental animal data. This apparent failure can be attributed to a number of different factors. These include the use of animal models that do not adequately represent clinical reality, e.g., due to lack of comorbidities; and poor study design (Ludman et al., 2010; Ovize et al., 2010; SchwartzLongacre et al., 2011; Hausenloy et al., 2010, 2013a; Bell et al., 2012).

More recently, several novel pharmacologic approaches have been reported to limit infarct size when administered before reperfusion in primary PCI-treated STEMI patients (Table 1). Most promising among these therapies are cyclosporine-A, exenatide, and metoprolol. Whether these pharmacologic postconditioning agents can actually improve clinical outcomes remains to be investigated, and in this regard, a large multicenter clinical outcome study is currently underway investigating cyclosporine-A (NCT 01502774).

In summary, mechanical and pharmacologic conditioning strategies are promising therapeutic options for cardioprotection in patients undergoing elective or emergency coronary revascularization, although there are several negative studies. Most of the clinical trials, both positive and negative, have been small. The positive trials have been conducted in selected patients under well controlled conditions, whereas the negative trials (e.g., on remote preconditioning) have been less selective in terms of patient recruitment and procedures (anesthesia, surgery). The observed lack of protection in the negative studies can in part be attributed to the presence of different risk factors, comorbidities, and their medications in different patient cohorts, as well as to poorly validated drug targets in juvenile and healthy animal models, and poorly designed clinical studies (Ferdinandy et al., 2007; Ovize et al., 2010; Hausenloy et al., 2013a). None of the existing studies has really raised a safety concern for the conditioning strategies. Larger studies with clinical outcome endpoints are necessary to gain more insight into the clinical applicability of conditioning strategies in different patient populations with different medications and confounding factors.

\section{Effects of Major Risk Factors on Ischemia/ Reperfusion Injury and Cardioprotective Strategies}

In the mid-1990s, hyperlipidemia was the first cardiovascular risk factor to be associated with the loss of preconditioning cardioprotection in rabbits and rats (Szilvassy et al., 1995; Ferdinandy et al., 1997). Since then, it has been well established that in addition to hyperlipidemia, most of the other major risk factors and/or medications that target them may modify cardioprotective signaling, leading to the loss or attenuation of cardioprotection by ischemic or pharmacologic conditioning (see for extensive earlier reviews: Ferdinandy et al., 1998, 2007; Ferdinandy, 2003). In this section, we review more recent evidence of the impact of the most important risk factors on ischemia/reperfusion injury and cardioprotection (Table 2).

\section{A. Aging and Cardioprotection}

1. Ischemic/Pharmacologic Preconditioning in Aging. Although ischemic and pharmacologic preconditioning attenuate ischemia/reperfusion injury in juvenile hearts, most studies suggest a loss of protection in aged hearts (for review, see Ferdinandy et al., 2007; Boengler et al., 2009; Przyklenk, 2011). By using endothelial function rather than myocardial infarct size as endpoint of protection in humans in vivo, increased age was associated with loss of protection by ischemic preconditioning against endothelial dysfunction after ischemia/ reperfusion in the brachial artery (van den Munckhof et al., 2013).

A number of studies have focused on different components of the signaling cascades (for review, see Heusch et al., 2008), assessing differences between young and aged hearts that might explain the observed loss of cardioprotection with aging.

a. Effect of aging on cardioprotective signaling.

i. Cytosolic Signaling. Blockade of the $\mathrm{Na}^{+} / \mathrm{H}^{+}$exchanger protected myocardium from ischemia/reperfusion injury in aged rats, whereas anesthetic preconditioning did not (Liu and Moore, 2010). cAMP-dependent protein kinase (PKA) activation and Akt activation are critical for ischemic preconditioning-induced cardioprotection (Yang et al., 2013). The adenylyl cyclase activator forskolin, which promotes subsequent PKA activation, reduced infarct size in young but not in aged rat hearts (Huhn et al., 2012). The loss of cardioprotection in aged, diabetic Goto-Kakizaki rats was associated with a chronic upregulation of Akt phosphorylation and a lack of further activation of Akt by ischemic preconditioning (Whittington et al., 2013b). The myocardial Akt isoforms Akt1 and Akt2 must be distinguished in their function for ischemic preconditioning's protection. The lack of a protective response to ischemic preconditioning in Akt1 knockout mice was accompanied by impaired phosphorylation (and thus inactivation) of GSK-3 $\beta$ (Kunuthur et al., 2012). Similarly, lack of pharmacologic preconditioning by isoflurane in aged rat hearts was associated with differences in the Akt/GSK-3 $\beta$ signaling pathway (Zhu et al., 2010), and pharmacologic GSK-3 $\beta$ inhibition decreased infarct size in young but not in old rat hearts 
TABLE 1

Clinical studies of PPCI-treated STEMI patients who reported beneficial effects with a pharmacologic agent administered at early reperfusion

\begin{tabular}{cccc}
\hline Clinical Study & Pharmacologic Postconditioning Agent & $\begin{array}{c}\text { Number of } \\
\text { Patients }\end{array}$ & Effect \\
\hline & $\begin{array}{l}\text { Atrial natriuretic peptide } \\
\text { J-WIND-ANP }\end{array}$ & 569 & $\begin{array}{c}\text { 15\% reduction in infarct size } \\
\text { (Kitakaze et al., 2007) } \\
\text { infusion started after } \\
\text { reperfusion }\end{array}$ \\
& $\begin{array}{c}\text { 2.0\% absolute increase in left } \\
\text { ventricular ejection fraction }\end{array}$
\end{tabular}

Piot et al., 2008

Lonborg et al., 2012

IMMEDIATE

(Selker et al., 2012)

$\begin{array}{cl}\text { METOCARD-CNIC 2013 } & \begin{array}{l}\text { Metoprolol } \\ \text { Intravenous metoprolol 3-5 mg } \\ \text { boluses administered in } \\ \text { ambulance before PPCI. }\end{array}\end{array}$

Intravenous CsA $(2.5 \mathrm{mg} / \mathrm{kg}) 10$ minutes before primary PCI

Exenatide started 15 minutes before primary PCI for 6 hours

Glucose insulin potassium (GIK) therapy

Intravenous GIK infusion for 12 ambulance before reperfusion
Intravenous infusion of exenatide hours started by paramedics in

357

Reduction in infarct size and less in-hospital mortality and cardiac arrest

220
58

44\% reduction in infarct size
( 72 -hour AUC total CK)
20\% reduction in infarct size
(CMR in subset of 27 patients)
28\% reduction in infarct size and
smaller LVESV on CMR at
6 months (Mewton et al., 2010)

107

Increase in myocardial salvage index at 90 days by CMR

Reduced infarct size as \% of AAR at 90 days by CMR

Patients presenting with short ischemic times ( $\leq 132 \mathrm{~min}$ ) had greater myocardial salvage
Mechanism of Cardioprotection

Atrial natriuretic peptide is a pharmacologic activator of a number of prosurvival signaling pathways including the RISK and cGMP-PKG pathways.

Cyclosporin-A is a known inhibitor of the mitochondrial permeability transition pore, a critical determinant of cardiomyocyte death.

Exenatide is a long-acting analog of GLP-1 that lowers blood glucose as well as limiting MI size through the activation of the RISK pathway.

AAR, area at risk measured; AUC, area under the curve; CK, creatinine kinase; CMR, cardiac MRI; LVESV, left ventricular end-systolic volume.

(Zhu et al., 2011a). In aged rat hearts, sirtuin deacetylase-1 (Sirt1) activity was increased after ischemia/reperfusion compared with young hearts (Adam et al., 2013). Although young Sirt1 knockout mice hearts could not be preconditioned (Nadtochiy et al., 2011b), a drug-induced increase in Sirt1 activity did not elicit cardioprotection after ischemia/reperfusion, suggesting that Sirt1 activity is necessary but not sufficient for the cardioprotective effects of ischemic preconditioning (Nadtochiy et al., 2011a) and is most likely not responsible for any observed age-related difference.

ii. Mitochondria. The different cytosolic signaling pathways activated by ischemic or pharmacologic preconditioning converge at the level of mitochondria (for review, see Heusch et al., 2008; Boengler et al., 2011a,b; Wojtovich et al., 2012), and the opening of certain mitochondrial ion channels alone is sufficient to elicit protection (for review, see Wojtovich et al., 2012). Pharmacologic preconditioning by helium, which protected young but not old rat hearts, could be abolished by blockade of the mitochondrial calcium-sensitive potassium channel (Heinen et al., 2008). As expected, pharmacologic activation of the mitochondrial calcium- sensitive potassium channel reduced irreversible injury induced by ischemia/reperfusion in young rat hearts but surprisingly was also effective in reducing infarct size in aged rat hearts (Huhn et al., 2012). GSK-3 $\beta$ inhibition significantly prolonged the time to MPTP opening induced by ROS in cardiomyocytes isolated from young but not from aged rat hearts (Zhu et al., 2011a, 2013b). Attenuation of ischemic or pharmacologic preconditioning's protection in the aged heart was associated with failure to reduce adenine-nucleotidetranslocase-cyclophilin-D interactions, a critical modulator of MPTP opening (Zhu et al., 2013b). Cyclosporine A, which binds cyclophilin D, thereby delaying MPTP opening, reduced myocardial infarct size and time to MPTP opening in young rats, whereas it failed to significantly affect either infarct size or time to MPTP opening in old rats (Liu et al., 2011b). Four weeks of treatment with the superoxide scavenger tempol restored pharmacologic preconditioning and cardioprotection by cyclosporine-A in old rats, and the reinstatement of the cardioprotected condition was associated with delayed onset of MPTP opening (Zhu et al., 2013a). 
TABLE 2

Effect of major risk factors on ischemia/reperfusion (I/R) injury as well as pre-, post-, and remote conditioning in the majority of the studies

\begin{tabular}{lcccc}
\hline \multicolumn{1}{c}{ Risk Factor } & I/R Injury & Preconditioning & Postconditioning & Remote Conditioning \\
\hline Aging & $\uparrow$ & $\downarrow$ & $\downarrow$ & - CL \\
Hypertension, hypertrophy, and remodeling & - & - & $\downarrow$ & N.D. \\
Hyperlipidemia & $\uparrow$ & $\downarrow$ CL & $\downarrow$ & N.D. \\
Diabetes & $\uparrow$ & $\downarrow$ CL & $\downarrow$ & $\downarrow$ CL \\
Uremia, kidney failure & $\uparrow$ & - & $\downarrow$ & N.D. \\
Impaired coronary microcirculation & $\uparrow \uparrow$ & $\downarrow$ & N.D.
\end{tabular}

CL, some clinical data are also available; N.D., no data available; $\uparrow$, enhance; $\downarrow$, attenuate,, , no effect.

b. Sex paradox. Most of the experimental studies (for review, see Ostadal et al., 2009) confirm the clinical observations (Canali et al., 2012) that female hearts have an increased resistance to ischemia/reperfusion injury, associated with an altered distribution of PKC and extracellular signal-regulated kinase (ERK) isoforms compared with male hearts (Hunter and Korzick, 2005). The already high tolerance of the adult female heart can be increased further by ischemic preconditioning. However, it seems that this protective effect of preconditioning in female animals depends on age: it was absent in the young female rat heart but it appeared with the decrease of resistance toward ischemia/reperfusion injury during aging (Ostadal et al., 2009). An increased resistance toward ischemia/reperfusion injury in aged female hearts could also be restored by a $\mathrm{PKC} \varepsilon$-activator administered before ischemia, and restoration of protection was associated with an enhanced mitochondrial PKC $\varepsilon$-translocation (Lancaster et al., 2011).

c. Delayed preconditioning (second window of protection). Twenty-four hour delayed anesthetic preconditioning with sevoflurane reduced infarct size in young but not in old rat hearts. Anesthetic preconditioning affected gene expression profiles (functional categories of cell defense/death, cell structure, gene expression/protein synthesis, inflammatory response/ growth/remodeling, and signaling/communication) of the cardiomyocyte in an age-associated pattern (Zhong et al., 2012).

2. Aging and Ischemic and Pharmacologic Postconditioning. Ischemic or pharmacologic postconditioning attenuates ischemia/reperfusion injury in young animal hearts (Skyschally et al., 2009). However, in most, although not all (Yin et al., 2009) studies, the protection is lost in aged hearts (for review, see Boengler et al., 2009; Przyklenk et al., 2011). Comparing ischemic preconditioning and postconditioning, one study suggested that ischemic postconditioning was less affected by aging than ischemic preconditioning (Vessey et al., 2009). However, genetic characteristics, a minor difference in age, or the number of postconditioning cycles are all critical factors for the successful effect of ischemic postconditioning and must be taken into consideration (Boengler et al., 2008a, 2009; Skyschally et al., 2009; Somers et al., 2011). Although there is no doubt that postconditioning protects human hearts (Heusch et al., 2013), there is some evidence that the extent of protection might depend on age. In a retrospective analysis, postconditioning the human heart by multiple balloon inflations failed to reduce irreversible injury in patients above the age of 65 years (Darling et al., 2007). By using the improvement of left ventricular function by postconditioning as endpoint, rather than reduction of infarct size, a recent meta-analysis also suggested a beneficial effect of postconditioning only in patients younger than 62 years (Zhou et al., 2012).

As with ischemic preconditioning, the more recent experimental studies have attempted to define specific alterations in the signaling mechanisms leading to the failure of protection by postconditioning in aged compared with young hearts.

a. Cytosolic signaling. Ischemic postconditioning reduced infarct size in young mice hearts, and the protection was associated with an upregulation of ERK but not Akt signaling. In contrast, postconditioning failed to limit infarct size in aged hearts, possibly as a consequence of the defect in ERK phosphorylation and increased mitogen activated protein kinase phosphatase-1 expression. Indeed, mitogen activated protein kinase phosphatase inhibition restored the ischemic postconditioned phenotype in aged mice hearts (Przyklenk et al., 2008). Similarly, pharmacologic postconditioning with isoflurane protected the heart in young but not in senescent rats; again the failure to activate the RISK pathway might have contributed to the attenuation of isoflurane-induced postconditioning effect in senescent rats (Chang et al., 2012). In one study with maintained reduction of infarct size by ischemic postconditioning in aged rat hearts $(16-18$ months), protection was accompanied by an increase in phosphorylation of Akt and GSK-3 $\beta$ similar to that measured in young rat hearts (Yin et al., 2009). In addition to the RISK pathway, the SAFE pathway also appears to be affected by age. The signal transducer and activator of transcription-3 (STAT3), which is involved in ischemia/reperfusion injury and cardioprotection by conditioning protocols (Boengler et al., 2008b), was less highly expressed and activated in aged mice hearts (Boengler et al., 2008a). Possibly, STAT3 plays a role in modifying mitochondrial function during ischemia/reperfusion, such as ROS formation (Boengler 
et al., 2013b) and opening of the MPTP (Boengler et al., 2010; Heusch et al., 2011). Although many postconditioning interventions are affected by age, pharmacologic postconditioning with sphingosine reduced infarct size to the same extent in young and aged rat hearts (Vessey et al., 2009). Blockade of PKG or PKA attenuated the cardioprotection by sphingosine, suggesting that the cyclic nucleotide-dependent signaling pathway utilized by sphingosine remains unaffected by age (Vessey et al., 2008a,b, 2009).

b. Mitochondria. Similarly to what has been described for ischemic preconditioning, direct pharmacologic inhibition of electron transport at reperfusion using amobarbital protected mitochondria and decreased myocardial injury in isolated aged rat hearts, even when signaling-induced pathways of postconditioning that are upstream of mitochondria were ineffective (Chen et al., 2012).

3. Aging and Remote Ischemic Preconditioning. Although remote ischemic preconditioning protects young and aged human hearts from ischemia/reperfusioninduced irreversible injury and improves patient outcomes after coronary artery bypass grafting, little is known about the age dependency of the process. In a recent experimental study, remote ischemic preconditioning by lower limb ischemia did not protect against ischemia/reperfusion injury in isolated newborn rabbit hearts and even caused deleterious effects in these hearts, although it effectively reduced infarct size in adult rabbit hearts (Schmidt et al., 2014).

Using endothelial function rather than irreversible myocardial injury as endpoint, healthy elderly people had a greater relative increase of flow-mediated vasodilatation after remote ischemic preconditioning than young individuals (Moro et al., 2011). Thus, whether an age dependency of remote ischemic preconditioning exists remains unknown at present.

Taken together, many studies demonstrate that protection by ischemic and pharmacologic preconditioning (early and delayed phase) and postconditioning is lost in aged hearts. Loss of cardioprotection is related to alterations in cytosolic signaling cascades leading to modification in the opening of MPTP. However, direct stimulation of mitochondrial targets might be capable of inducing protection even in aged hearts.

\section{B. Hypertension, Cardiac Hypertrophy, and Remodeling}

Ischemic or pharmacologic (e.g., adenosine-receptor agonist, propofol) preconditioning reduced infarct size in normotensive and hypertensive, hypertrophied rat hearts in vitro (Ebrahim et al., 2007; Hochhauser et al., 2007; King et al., 2012) and in vivo (Dai et al., 2009). Similarly, pharmacologic preconditioning with isoflurane 6 weeks after permanent coronary artery ligation reduced infarct size after ischemia/reperfusion in the remaining myocardium, although hearts exhibited a substantial compensatory hypertrophy. The cardioprotection by isoflurane was abolished by inhibition of phosphatidylinositol 3-kinase (PI3K) or $\mathrm{K}_{\mathrm{ATP}}$ blockade, indicating that the established signaling cascade of protection was intact in the remodeled myocardium (Lucchinetti et al., 2008). In contrast, ischemic postconditioning reduced infarct size in normotensive but not hypertensive rat hearts (Penna et al., 2010; Wagner et al., 2013). The phosphorylation of GSK- $\beta$ was increased by ischemic postconditioning in normotensive rats. However, this increase was completely absent in hypertensive, hypertrophied rat hearts (Wagner et al., 2013). In anabolic steroid-induced cardiac hypertrophy, ischemic postconditioning failed to reduce infarct size after ischemia/reperfusion; postconditioning increased Akt phosphorylation regardless of its protective effects, but reduced expression of protein phosphatase expression was measured in protected hearts (Penna et al., 2011). Chronic captopril treatment significantly reduced left ventricular hypertrophy in hypertensive rats and reduced infarct size after ischemia/reperfusion in isolated hearts from both normotensive and hypertensive rats. Ischemic postconditioning was unable to add its protective effect to that of chronic captopril although treatment induced hypertrophy regression and almost completely normalized left ventricular pressure (Penna et al., 2010).

Taken together, although preconditioning's protection is still present in animals with hypertension and/or left ventricular hypertrophy, infarct size reduction by ischemic postconditioning appears to be lost. Once again the lack of protection by ischemic postconditioning relates to changes in the cytosolic signaling pathway. Treatment of the primary disease (hypertension, hypertrophy) does not restore the cardioprotection by ischemic postconditioning.

\section{Hyperlipidemia and Atherosclerosis}

1. Ischemia /Reperfusion Injury, Ischemic Pre-, Post-, and Remote Conditioning in Hyperlipidemia. In epidemiological studies, there is a well recognized relationship between serum total cholesterol concentration and the morbidity and mortality due to MI. Previously, this was attributed solely to the development of coronary atherosclerosis as a result of hypercholesterolemia. However, in the last two decades, a significant volume of evidence has accumulated showing that hyperlipidemia exerts direct effects on the myocardium that may interfere with cardioprotective mechanisms. Although there are some conflicting results, most of the preclinical studies, together with some small scale clinical studies, have shown that hyperlipidemia per se, but not atherosclerosis, leads to a significant aggravation of myocardial ischemia/reperfusion injury and to attenuation of the cardioprotective effect of both early and late preconditioning. These studies were reviewed by us previously (Ferdinandy et al., 2007). 
More recent studies confirm the deleterious effects of hyperlipidemia on susceptibility to ischemia/reperfusion injury and on ischemic preconditioning. Expansion of infarct size in a hyperlipidemic pig model was shown by Osipov et al. (2009). The loss of the infarct size limiting effect of ischemic preconditioning (Gorbe et al., 2011; Landim et al., 2013; Babbar et al., 2013; Yadav et al., 2010b; Xu et al., 2013) and late ischemic preconditioning (Yadav et al., 2012) have been shown in different models of diet-induced hyperlipidemia in rats.

The loss of the infarct size limiting effect of ischemic postconditioning has been also confirmed in the last 5 years by several studies in hypercholesterolemic rat (Kupai et al., 2009; Landim et al., 2013; Wu et al., 2014) and rabbit models (Andreadou et al., 2012; Iliodromitis et al., 2010). In the fructose-fed hypertriglyceridemic rat heart, cholesterol lowering by fenofibrate restored ischemic preconditioning-mediated cardioprotection (Babbar et al., 2013). However, in one study ischemic postconditioning was still effective in hyperlipidemic rats (Zhao et al., 2009a). The effect of hyperlipidemia on remote conditioning has not yet been reported.

2. Pharmacologic Cardioprotection in Hyperlipidemia: Implications for Target Validation. One of the most extensively investigated cardioprotective cellular signaling pathways is the NO-cGMP-PKG pathway, and elements of this signaling pathway are thought to be promising drug targets (Burley et al., 2007; GarciaDorado et al., 2009; Gorbe et al., 2010; Andreadou et al., 2014; Bice et al., 2014). However, Giricz et al. (2009) showed that, although the NO donor SNAP, which activates soluble guanylate cyclase, or BNP, which activates particulate guanylate cyclase, or the stable cGMP analog 8-bromo cGMP, significantly reduced infarct size in normal rat hearts, none of these treatments was effective in hearts of rats fed a cholesterolenriched diet. The loss of cardioprotection by activators of the cGMP-PKG pathway was possibly due to inactivation of $\mathrm{PKG}$ by oxidative dimerization of the kinase under hyperlipidemic conditions. These results indicate that drug targets upstream of PKG are less likely targets for cardioprotection, because activation of such targets does not provide cardioprotection in the presence of hyperlipidemia. Another recognized cardioprotective pathway includes $\mathrm{K}_{\mathrm{ATP}}$ channel activation. However, it was recently shown that the infarct size limiting effect of either the nonselective $\mathrm{K}_{\mathrm{ATP}}$ activator cromakalim or the selective mitochondrial $\mathrm{K}_{\mathrm{ATP}}$ activator diazoxide was abrogated in hyperlipidemic, cholesterolfed rats (Csonka et al., 2014). Sevoflurane-induced delayed cardioprotection against ischemia/reperfusion injury was also lost in hypercholesterolemia, potentially via interference of hyperlipidemia with the inducible NO synthase/mitochondrial $\mathrm{K}_{\mathrm{ATP}}$ channel pathway in rats (Zhang et al., 2012). Moreover, in hyperlipidemic Zucker obese rats, neither postconditioning nor the
MPTP inhibitor cyclosporine-A could exert cardioprotection (Huhn et al., 2010). These results indicate that activation of the NO-cGMP-PKG pathway or $\mathrm{K}_{\mathrm{ATP}}$ channels and inhibition of the MPTP may not be ideal cardioprotective drug targets, because they are ineffective in the presence of hyperlipidemia, a frequent attendant condition in patients with ischemic heart disease.

In contrast, the use of hyperlipidemic animal models of cardioprotection reveals some pharmacologic targets that remain effective in the presence of hyperlipidemia. Cardioprotection by preconditioning involves an approximately $20 \%$ inhibition of myocardial MMP2 (Lalu et al., 2002; Giricz et al., 2006). However, MMP2 inhibition by preconditioning is absent in hyperlipidemic rats. Giricz et al. (2006) showed that by mimicking the moderate MMP2 inhibitory effect of preconditioning with the inhibitor ilomastat, cardioprotection occurred in both normal and hyperlipidemic hearts. Effective cardioprotection by a moderate inhibition of intracellular MMP2 was confirmed recently by Bencsik et al. (2014). MMP2 inhibition as a pathway parallel to the known cardioprotective pathways has been confirmed by Bell et al. (2013). Dietary supplementation of red palm oil in rats with established hyperlipidemia was able to reduce infarct size associated with MMP2 inhibition (Szucs et al., 2011). These results show that MMP2 inhibition might be a valid cardioprotective target, because moderate inhibition of MMP2 in the heart confers cardioprotection in both normal and hyperlipidemic animals subjected to MI (Dorman et al., 2010). Independent of MMP2 inhibition, Yadav et al. (2012) showed that pharmacologic inhibition of GSK- $3 \beta$ produced a late cardioprotected state in both normal and hyperlipidemic rats, possibly associated with $72 \mathrm{kDa}$ heat shock protein induction. This may also suggest an MPTP-independent effect of GSK-3 $\beta$ inhibition.

3. Effect of Hyperlipidemia on Cardioprotective Cellular Mechanisms. The mechanism by which hyperlipidemia may influence the severity of myocardial ischemia/reperfusion injury and cardioprotection is not fully understood. However, decreased cardiac NO content, increased oxidative/nitrosative stress, inactivation of PKG, impairment of the mevalonate pathway, decreased heat-shock response, increased ecto-5'nucleotidase activity, enhanced apoptotic cell death, as well as dramatic changes in cardiac gene expression profile, have all been demonstrated as consequences of hyperlipidemia (see for earlier reviews: Ferdinandy et al., 1998, 2007; Ferdinandy, 2003). Moreover, there is evidence that the microRNA expression pattern of hyperlipidemic rat hearts (Varga et al., 2013) and the gene expression patterns in the Zucker Diabetic Fatty rat strain are changed (Sarkozy et al., 2013), indicating that the heart responds to the systemic hyperlipidemic state by concerted alterations in gene expression 
including genes controlling metabolic functions. We also showed that hyperlipidemia changes $\mathrm{Cx} 43$ distribution in the sarcolemma and in the mitochondria (Gorbe et al., 2011). Hyperlipidemia also interferes with the nitrosative triggering signal of postconditioning in rats (Kupai et al., 2009)

In summary, the majority of preclinical studies show that hyperlipidemia worsens the outcome of ischemia/ reperfusion injury and attenuates the cardioprotective effect of both early and late preconditioning, postconditioning, and pharmacologic conditioning via hyperlipidemiainduced changes in cardioprotective signaling pathways. Nevertheless, there are some promising targets that may be still effective in the hyperlipidemic heart, e.g., MMP-2. These findings emphasize the necessity for the development of new cardioprotective drugs that are able to reverse the increased susceptibility of hyperlipidemic hearts to ischemia/reperfusion stress and to enhance adaptive cardioprotective mechanisms in hyperlipidemic patients.

\section{Diabetes}

Epidemiological studies and clinical trials have clearly shown that both type I (insulin-dependent) and type II (noninsulin-dependent) diabetics are more prone to developing ischemic heart disease, including acute MI and post-MI complications (Otto et al., 2012; Baars et al., 2013). The diabetic heart is more susceptible to acute myocardial ischemia/reperfusion injury (Alegria et al., 2007; Marso et al., 2007).

1. Ischemia/Reperfusion Injury in Diabetes. Although it has long been known that diabetes is an independent risk factor for the development of ischemic heart disease and that the long-term outcome of ischemic heart disease is worsened by diabetes in humans, inconsistency exists in the literature regarding the susceptibility of myocardium to acute ischemia/reperfusion injury in various animal models of diabetes (reviewed in Ferdinandy et al., 2007; Miki et al., 2012; Whittington et al., 2012). With respect to the preclinical animal studies, it appears that the susceptibility of the diabetic heart to acute ischemia/reperfusion injury is critically dependent on the duration and severity of the diabetes model and the experimental conditions of the acute ischemia/reperfusion injury model.

In experimental studies, the diabetic heart has been shown to be less sensitive to acute ischemia/reperfusion injury in those experimental studies that used 1) a short duration of diabetes ( $<6$ weeks); 2 ) glucose as the only substrate; and 3) a no-flow acute ischemia/reperfusion injury protocol. In contrast, in studies where the diabetes model was more prolonged and severe, fatty acids were present in the substrate, and a low-flow acute ischemia/reperfusion injury protocol was used, the diabetic heart was found to be more sensitive to acute ischemia/reperfusion injury (Miki et al., 2012; Whittington et al., 2012). In contrast to the inconsistent preclinical studies, the majority of clinical studies demonstrates worse outcome from acute MI in diabetic patients. This suggests that the use of chronic diabetes animal models better reflects the clinical situation.

A number of studies have examined cardioprotection in different experimental animal models of diabetes and in diabetic patients. The majority of studies shows that the presence of diabetes may interfere with the cardioprotective mechanisms, attenuating the effectiveness of these therapeutic strategies. As expected in some of the animal diabetic models used in the experimental studies, there is some overlap with the related comorbidities of obesity and metabolic syndrome, both of which may also impact on the efficacy of cardioprotection. Many of the therapies used to treat diabetes may also impact on the cardioprotective intervention (discussed in a later section V.C). Finally, some experimental studies are beginning to investigate the effect of one or more comorbidities on the efficacy of cardioprotective strategies, which better reflects the clinical setting in which multiple comorbidities often coexist with ischemic heart disease.

2. Cardioprotection by Preconditioning in Diabetes. The majority of preclinical experimental studies investigating the effect of diabetes on the myocardial response to either ischemic or pharmacologic preconditioning has found that the presence of diabetes renders the heart more resistant to the infarct size-limiting effects of preconditioning (reviewed in Ferdinandy et al., 2007; Miki et al., 2012). Recent experimental studies have also shown that the cardioprotective efficacy of pharmacologic preconditioning using a variety of different agents, including erythropoietin (Miki et al., 2009; Hotta et al., 2010), $\delta$-opioid receptor agonist (Hotta et al., 2010), isoflurane (Matsumoto et al., 2009), L-glutamate (Povlsen et al., 2009), remifentanil (Kim et al., 2010), and helium (Huhn et al., 2009b), is also impaired in the diabetic heart. Whittington et al. (2013b) investigated the combined effect of diabetes and age on the response of the heart to acute ischemia/ reperfusion injury. As expected, the combination of aging (up to 18 months in the rat) and diabetes (Goto-Kaziaki rat) increased infarct size in response to acute ischemia/reperfusion and raised the threshold for ischemic preconditioning in a predictably additive manner.

a. Mechanisms contributing to resistance of the diabetic heart to preconditioning. A variety of different mechanisms have been suggested to contribute to the impaired response of the diabetic heart to preconditioning. These include impaired activation of known intracellular prosurvival signaling pathways, such as the Akt and ERK1/2 components of the RISK pathway (Hausenloy and Yellon, 2007), and its downstream targets, such as GSK-3 $\beta$ and the Janus-activated kinaseSTAT3 components of the SAFE pathway (Lecour, 2009). More recent experimental studies have investigated the effect of chronic Akt activation on the myocardial 
response to preconditioning. Whittington et al. (2013b) found that in the aged rat heart (12-18 months old) there was chronic activation of Akt, which was not further augmented in response to ischemic preconditioning, suggesting that in this setting Akt activation may not mediate cardioprotection. In this regard, Fullmer et al. (2013) showed that pharmacologic (insulin) or genetic activation of Akt in the heart blocked the infarct size-limiting effects of ischemic preconditioning.

Since 2007, experimental studies have investigated several novel mechanisms to explain the impaired response of the diabetic heart to preconditioning. Miki et al. (2009) reported that the presence of augmented endoplasmic reticulum stress in the diabetic heart blocked ERK1/2-mediated phosphorylation of GSK-3 $\beta$, leading to increased susceptibility to MPTP opening and mitochondrial calcium overload. Hotta et al. (2010) showed that the angiotensin II subtype 1 receptormediated upregulation of calcineurin in the diabetic rat heart interfered with the phosphorylation of Janusactivated kinase 2 and PI3K-Akt signaling, thereby affecting the efficacy of pharmacologic preconditioning. Ajmani et al. (2011) provided data implicating caveolin, which was increased in the diabetic heart and interfered with endothelial NO synthase (eNOS) activity, contributing to the inability of the diabetic heart to respond to ischemic preconditioning. Impaired mitochondrial biogenesis, secondary to a dysfunctional adiponectinadenosine monophosphate-activated kinase (AMPK) axis (Yan et al., 2013), and increased $\mathrm{TNF} \alpha$-induced oxidative stress (Su et al., 2013) were also proposed as mechanisms underlying the greater sensitivity of the diabetic heart to ischemia/reperfusion injury and its resistance to ischemic preconditioning. A recent study by Gurel et al. (2013) provided preliminary data suggesting that in the diabetic heart the failure of ischemic preconditioning to localize hexokinase to mitochondria may contribute to the lack of cardioprotection. Finally, an intriguing study by Vinokur et al. (2013) suggests that altered iron metabolism may contribute to the response of the diabetic heart to acute ischemia/reperfusion injury and ischemic preconditioning. Under basal conditions diabetic hearts sustained less acute ischemia/reperfusion injury, a finding that was associated with basally increased levels of myocardial ferritin, a cardioprotective factor. Conversely, the failure of the diabetic heart to respond to ischemic preconditioning was found to be associated with an accelerated loss of myocardial ferritin during ischemia.

To overcome the resistance of the diabetic heart to preconditioning, several studies have used pharmacologic agents to target the effectors of cardioprotection downstream of the impaired signaling pathways or have used pharmacologic modulation of alternative cardioprotective pathways. In this regard, it has been shown that pharmacologic inhibition of GSK- $3 \beta$, a downstream target of Akt and ERK1/2, was able to reduce infarct size in the diabetic heart (Miki et al., 2009; Yadav et al., 2010a). Other experimental studies have reported being able to pharmacologically precondition the diabetic heart using a variety of diverse agents including olprinone (a phosphodiesterase type 3 inhibitor) (Matsumoto et al., 2009) and even ginsenoside $\mathrm{Rb} 1$ (a pharmacologically active component of ginseng) (Wu et al., 2011b).

b. Restoring myocardial sensitivity to preconditioning in the presence of diabetes. Whether antidiabetic therapy or another pharmacologic agent can restore the sensitivity of the diabetic heart to preconditioning has been investigated. $\mathrm{Gu}$ et al. (2008) found that simvastatin treatment was able to restore cardioprotection elicited by ischemic preconditioning in the presence of hyperglycemia, and this effect was associated with the generation of NO. Pretreatment of diabetic Goto-Kakizaki rats with the antidiabetic sulfonylurea glimepiride (which did not reduce infarct size itself) was demonstrated to restore the sensitivity of the myocardium to ischemic preconditioning, such that one cycle instead of three cycles of ischemic preconditioning was sufficient to limit infarct size (Hausenloy et al., 2013c). However, this effect of glimepiride in lowering the threshold for ischemic preconditioning appeared to be independent of serum glucose levels, because the latter remained unchanged with glimepiride treatment (Hausenloy et al., 2013c). Finally, chronic insulin treatment increases cardiac adiponectin and restores cardioprotective AMPK signaling (Pei et al., 2013).

Experimental studies using human right atrial tissue harvested from patients undergoing coronary artery bypass grafting surgery have reported that myocardial tissue from diabetic patients is resistant to ischemic preconditioning when subjected to simulated acute ischemia/reperfusion injury (Ovunc, 2000; Barua et al., 2011). Barua et al. (2011) showed that the resistance to ischemic preconditioning could be overcome by modulating NO availability in atrial tissue from diabetic patients. Interestingly, Sivaraman et al. (2010) demonstrated that intensifying the ischemic preconditioning stimulus protected isolated human right atrial trabeculae harvested from diabetic patients against simulated acute ischemia/reperfusion injury. In this study, the inability to precondition the diabetic atrial tissue using a standard preconditioning protocol was associated with lower basal levels of Akt activation (Sivaraman et al., 2010). Furthermore, Wang et al. (2011b) reported enhanced expression of PTEN (a suppressor of Akt activity) through microRNA interference (Ling et al., 2013) associated with reduced Akt and downstream eNOS expression in diabetic human atrial tissue.

In vivo human data have confirmed the resistance of diabetic patients to the protective effect of ischemic preconditioning. Engbersen et al. (2012) demonstrated using a forearm model of endothelial injury that the 
efficacy of ischemic preconditioning to reduce acute ischemia/reperfusion injury was lower in type $1 \mathrm{di}$ abetic patients and was completely abolished in these patients during hyperglycemia.

3. Cardioprotection by Postconditioning in Diabetes and Metabolic Syndrome. Experimental animal data suggest that the presence of diabetes and related conditions, such as obesity and metabolic syndrome, may impact on the cardioprotective efficacy of both ischemic and pharmacologic postconditioning. For ischemic postconditioning, using either a genetic or a chemically induced murine model of diabetes, Przyklenk et al. (2011) found that the isolated perfused diabetic heart was resistant to the infarct size-limiting effects of ischemic postconditioning compared with the normoglycemic heart. In this study, the lack of response to ischemic postconditioning in the diabetic heart was associated with a failure to activate the ERK1/2 component of the RISK pathway. By using an isolated rat heart model of acute ischemia/reperfusion injury, Ren et al. (2011) demonstrated that the failure to postcondition the diabetic heart appeared to be linked to reduced myocardial expression of transient receptor potential vanilloid 1 channel, calcitonin gene related peptide, and substance $\mathrm{P}$ (Babiker et al., 2012) have shown that the diabetic heart was resistant to a novel form of postconditoning using pacing (10 cycles of 30 seconds left ventricular pacing alternated with 30 seconds right atrial pacing) compared with the nondiabetic heart.

Further studies have demonstrated that pharmacologic postconditioning using a variety of agents can also be affected by the presence of diabetes. Raphael et al. (2010) found that hyperglycemia abrogated the cardioprotection elicited by the inhaled anesthetic isoflurane, administered at the onset of myocardial reperfusion, using a rabbit in vivo model of acute ischemia/reperfusion injury, a finding that was associated with impaired activation of Akt-eNOS. Similarly, the infarct size-limiting effect of the inhaled anesthetics desflurane (Tai et al., 2012) and sevoflurane (Drenger et al., 2011), administered at the onset of myocardial reperfusion to rats in vivo, was abolished in the presence of diabetes. This was associated with a failure of these anesthetic agents to activate Akt, ERK1/2, GSK-3 $\beta$ (Tai et al., 2012), and STAT3 (Drenger et al., 2011). An interesting study by Potier et al. (2013) suggested that there may be differential effects in the response to cardioprotection using different bradykinin receptor agonists in the presence of diabetes. It was shown that ischemic postconditioning, the angiotensin-converting enzyme (ACE) inhibitor ramiprilat or a bradykinin $B_{2}$ receptor agonist, all reduced infarct size and activated Akt, ERK1/2, and GSK- $3 \beta$ in the nondiabetic heart but failed to do so in the diabetic heart (Potier et al., 2013). In contrast, the diabetic heart, but not the nondiabetic heart, was found to be amenable to pharmacologic postconditioning using a bradykinin $\mathrm{B}_{1}$ receptor agonist (Potier et al., 2013).
A number of experimental studies have investigated the cardioprotective effects of combining ischemic and pharmacologic postconditioning in the diabetic heart. Badalzadeh et al. (2012) demonstrated that the isolated diabetic rat heart was resistant to cardioprotection by either ischemic postconditioning or cyclosporine-A alone, but with the combination of interventions, a significant reduction in infarct size was observed. In contrast, Fan et al. (2012) found that atorvastatin was able to postcondition the diabetic heart, despite it being resistant to ischemic postconditioning; when given in combination, an additive cardioprotective effect was seen. These data suggest that the diabetic heart may be amenable to cardioprotection if the intensity of the postconditioning stimulus is enhanced.

Attempts have been made to recapture the cardioprotective effects of postconditioning by correcting the hyperglycemic state. In this regard, although insulin pretreatment failed to restore the sensitivity of the diabetic heart to postconditioning (Drenger et al., 2011), the transplantation of pancreatic islet tissue was able to reverse the effect of diabetes on the myocardial response to postconditioning (Przyklenk et al., 2011).

In contrast to the above findings, Lacerda et al. (2012) found that despite the presence of diabetes, the murine heart was equally sensitive to ischemic postconditioning $(6 \times 10$-second cycles of coronary arterial occlusion/reperfusion) as the nondiabetic heart. However, this discordant finding may in part be explained by the fact that the streptozotocin-induced diabetes was only initiated 5-10 days before MI. A recent study by Oosterlinck et al. (2013), using a genetic murine model of diabetes (ob/ob and DKO strains), demonstrated that the cardioprotective effect of ischemic postconditioning was attenuated, although this may in part relate to the use of a nonstandard ischemic postconditioning protocol $(3 \times 10$-second cycles of coronary arterial occlusion/reperfusion).

Bouhidel et al. (2008) investigated the effect of obesity alone on the efficacy of ischemic postconditioning. They found that the leptin-deficient obese (ob/ob) mice were resistant to cardioprotection by ischemic postconditioning and that this was associated with an inability to activate Akt, ERK1/2, p70S6 kinase, and AMPK. By using a rat model of the metabolic syndrome, Wagner et al. (2008) found that the Wistar-Ottawa-Karlsburg W rats were resistant to the cardioprotection elicited by ischemic postconditioning, and this was associated with an inability to phosphorylate ERK1/2 and its downstream effector GSK-3 $\beta$. Huhn et al. $(2009 \mathrm{~b}, 2010)$ reported that the Zucker obese rat was resistant to pharmacologic postconditioning using either sevoflurane or cyclosporine-A. The finding that the presence of a comorbidity could impact on the cardioprotective efficacy of cyclosporine-A is somewhat surprising, because its target, the MPTP, is believed to be downstream of the signaling pathways affected by the comorbidity. 
However, one can speculate that the comorbidity may interfere with cardioprotection by modulating mitochondrial function. An interesting study by Pons et al. (2013) showed that regular treadmill exercise was able to protect the heart in obese (ob/ob) mice through the activation of prosurvival kinases (Akt, ERK1/2, GSK-3 $\beta$, p70S6 kinase, AMPK) and in the absence of any improvement in metabolic profile. These findings suggest that regular exercise may have beneficial effects on the heart independently of any effect it may have on the metabolic profile.

Despite the preclinical data demonstrating the resistance of the diabetic heart to both ischemic and pharmacologic postconditioning, there have been no clinical trials investigating the effect of diabetes on the cardioprotective efficacy of postconditioning. This is despite a number of proof-of-concept clinical studies comprising a significant proportion of diabetic patients, reporting beneficial effects of postconditioning in STEMI and coronary artery bypass graft surgery patients (see section III). Interestingly, Lemoine et al. $(2008,2010,2011)$ found that human atrial trabeculae subjected to simulated acute ischemia/reperfusion injury were equally sensitive to the cardioprotective effects of pharmacologic postconditioning using desflurane, whether harvested from diabetic or nondiabetic patients undergoing coronary artery bypass graft surgery.

4. Cardioprotection by Remote Ischemic Conditioning in Diabetes. Despite the clinical potential of remote ischemic conditioning, there is a paucity of animal data investigating whether the diabetic heart is amenable to this cardioprotective intervention. Investigating the effect of diabetes in this setting can be quite challenging, given that the presence of diabetes may modulate signaling at any one or more of the following stages of the mechanistic pathway: the remote organ or tissue in which the conditioning stimulus is applied, the neurohormonal pathway that conveys the cardioprotective stimulus to the heart, and finally, the activation of intracellular prosurvival signaling pathways within the heart (reviewed in Hausenloy and Yellon, 2008).

There has been only one experimental animal study published specifically investigating the cardioprotective efficacy of remote ischemic preconditioning in the presence of diabetes. Zhu et al. (2011b) found that the diabetic rat heart in vivo was amenable to infarct size reduction elicited by limb preconditioning $(3 \times 5$ minute cycles of hindlimb cuff inflation/deflation). However, it is important to note that in this study streptozotocin-induced diabetes was only initiated 1 week before MI. Whether remote ischemic preconditioning would have been effective in a chronic diabetes animal model remains to be determined.

Remote ischemic conditioning, using a cuff placed on either the upper arm or leg to induce three or more cycles of brief ischemia and reperfusion, has been reported to have beneficial effects in a number of clinical settings including STEMI patients treated by primary PCI, coronary artery bypass graft patients, and patients undergoing elective PCI (reviewed in Hausenloy and Yellon, 2008; Candilio et al., 2011; Heusch, 2013). Although many of these clinical studies included diabetic patients they did not specifically address the question of whether the diabetic heart was amenable to remote ischemic conditioning.

$\mathrm{Xu}$ et al. (2014) found that a standard remote ischemic conditioning stimulus $(3 \times 5$-minute upper arm cuff inflations/deflation) did not reduce the magnitude or incidence of periprocedural myocardial injury during elective PCI in older patients (mean age 69 years) with diabetes. However, it is difficult to distinguish the effect of diabetes from that of age on the remote ischemic preconditioning protocol. Furthermore, the authors of this study did not demonstrate that their remote ischemic preconditioning protocol was effective in a younger nondiabetic population (Xu et al., 2014). In an intriguing clinical study by Jensen et al. (2012) it was reported that diabetic patients with a peripheral neuropathy failed to produce a cardioprotective humoral factor in response to a standard remote ischemic preconditioning protocol $(4 \times 5$-minute upper arm cuff inflation/deflation) compared with nondiabetic and diabetic patients who did not have a peripheral neuropathy. This finding supports the notion that an intact neural pathway from/to the remotely conditioned organ or tissue is required to elicit the cardioprotective humoral factor (Jensen et al., 2012). In this particular study, the cardioprotective efficacy of the humoral factor was tested in an isolated perfused rabbit heart ischemia/reperfusion model, and therefore whether the diabetic heart was amenable to remote ischemic preconditioning cardioprotection was not actually investigated (Jensen et al., 2012). A later study by the same research group investigated the impact of myocardial $O$-linked $\beta$ - $N$-acetylglucosamine $(O$-GlcNAc) in remote ischemic preconditioning-induced protection (Jensen et al., 2013). They found that plasma dialysate harvested from either normal volunteers or diabetic patients treated with a standard protocol of remote ischemic preconditioning $(3 \times 5$ cycles of upper am cuff inflation/deflation) was able to protect naive human right atrial trabeculae subjected to simulated ischemia/ reperfusion injury, findings which were associated with increased levels of myocardial $O$-GlcNAc (Jensen et al., 2013). These findings appear to suggest that increased myocardial levels of $O$-GlcNAc may indicate a cardioprotective phenotype.

In summary, the majority of both preclinical and clinical data suggests that the diabetic heart is more susceptible to ischemia/reperfusion injury and that the cardioprotective effect of ischemic and pharmacologic preconditioning and of postconditioning is impaired in the presence of diabetes. This impairment appears to be associated with deficient activation of prosurvival 
signaling pathways, such as Akt, ERK1/2, AMPK, and STAT3. Due to the limited preclinical and clinical studies available, whether the diabetic heart is amenable to remote ischemic conditioning is currently not known.

\section{E. Kidney Failure and Uremia}

Uremia, resulting from kidney failure, is a metabolic disease associated with a high prevalence of ischemic heart disease (Levey and Coresh, 2012). Therefore, one may speculate that a uremic state may also lead to the attenuation of endogenous cardioprotective mechanisms. However, Byrne et al. (2012) reported that ischemic preconditioning, remote conditioning, and postconditioning were still cardioprotective after 4 weeks of subtotal nephrectomy or adenine-enriched diet-induced acute uremia in rats. They also showed in this model that the RISK and SAFE signaling pathways were unaffected. However, experimental models of acute uremia may not properly reflect the clinical situation, because uremia frequently remains unrecognized until its late stages (Levey and Coresh, 2012). Kocsis et al. (2012) studied if prolonged experimental uremia of 30 weeks duration affects ischemic preconditioning. They found that although prolonged experimental uremia led to severe metabolic changes and mild myocardial dysfunction, the cardioprotective effect of ischemic preconditioning was still preserved 30 weeks after partial nephrectomy in rat hearts. However, it is not known if longer durations of uremia may interfere with postconditioning or remote conditioning. Human conditioning studies in uremia have not been reported.

Taken together, the limited preclinical studies suggest that despite the complex systemic metabolic changes in uremia, cardioprotection by pre- and postconditioning is preserved. Nevertheless, further preclinical studies in long-term experimental uremia models, as well as clinical studies, will be necessary to show if mechanical or pharmacologic conditioning can still protect the heart in uremic patients.

\section{F. The Diseased Coronary Circulation}

Experimental studies on myocardial ischemia/ reperfusion are usually performed in healthy young animals; moreover, irrespective of age, these animals have a virgin coronary circulation. In clinical reality, atherosclerosis develops progressively over time. Plaque fissure and rupture are then acutely superimposed on the underlying atherosclerosis and further complicated by intraluminal platelet aggregation and coagulation. In contrast, experimental studies usually rely on abrupt closure and reopening of the epicardial coronary artery with external devices. Therefore, the status of both the epicardial coronary arteries and the coronary microcirculation is vastly different between clinical reality and most experimental models. We need to consider these differences as confounders in translation of cardioprotective strategies (Heusch et al., 2012a). In fact, in most but not all of the more clinically relevant conditions, a diseased coronary circulation tends to attenuate the efficacy of cardioprotection.

1. Epicardial Coronary Arteries. Atherosclerotic plaque rupture in an epicardial coronary artery with superimposed intraluminal thrombotic occlusion of the coronary arterial lumen is the culprit event that causes acute MI in the clinical setting. Primary PCI removes the occlusive thrombus but-unless there is direct stenting-leaves the underlying atherosclerotic vascular lesion unaffected. Therefore, reperfusion does not occur through a fully patent epicardial coronary artery but one with a residual stenosis. As a consequence, there is not abrupt, but attenuated, more gentle reperfusion. This gentle reperfusion will be even more prominent when thrombolysis is applied, giving slow thrombus dissolution. Gentle reperfusion per se attenuates reperfusion injury and reduces infarct size (Heusch, 2004), as has been demonstrated by gradual restoration of coronary perfusion pressure in isolated rodent heart preparations (Nemlin et al., 2009) and slow restoration of coronary blood flow in larger mammals in vivo (Musiolik et al., 2010). Therefore, when comparing a group of patients undergoing a conditioning intervention to a purported control group, which inadvertently undergoes gentle reperfusion through a residual stenosis and thereby experiences some protection, the difference between the two groups that reflects the magnitude of cardioprotection by the conditioning intervention is diminished. Use of direct stenting removes any residual stenosis and coronary microembolization from the culprit lesion such that the consequences of immediate full reperfusion in the control group are compared with those of the postconditioning algorithm in the intervention group (Loubeyre et al., 2002; Heusch, 2012).

2. The Coronary Microcirculation. Plaque rupture is not only the causal index event that leads to acute MI; minor plaque fissure or rupture may also precede or follow the index event, initiated either spontaneously and/or after mechanical intervention. In the absence of coronary occlusion, plaque fissure/rupture releases particulate debris from the underlying atherosclerotic lesion that is superimposed by intraluminal platelet aggregates and coagulation material. These atherothrombotic particles are then washed into the coronary microcirculation where they cause microembolization. Coronary microembolization with inert particles in dogs and pigs induces microinfarcts and an inflammatory reaction (Heusch et al., 2009). However, in patients the ruptured atherosclerotic plaque also releases soluble vasoconstrictor, proinflammatory and prothrombotic mediators that contribute to the impairment of coronary microvascular perfusion (Kleinbongard et al., 2011). Both the particulate debris and the soluble mediators 
not only impair coronary microvascular perfusion per se but may also interfere with cardioprotective conditioning strategies.

As discussed previously (section III.A), preinfarction angina is associated with cardioprotection and probably represents a clinical correlate of ischemic preconditioning. In a pig model, coronary microembolization, which may occur clinically during preinfarction angina, induces microinfarcts that add to the aggregate infarct size caused by the index MI. Coronary microembolization with inert particles in pigs neither induces acute ischemic preconditioning nor does it interfere with acute ischemic preconditioning (Heusch et al., 2009). However, on a somewhat more prolonged time frame, coronary microembolization causes the upregulation of $\mathrm{TNF} \alpha$ over several hours, which then induces cardioprotection (Skyschally et al., 2007); this form of protection several hours after coronary microembolization was termed a "third window of ischemic preconditioning" (Heusch et al., 2007).

The no-reflow phenomenon is the most severe form of coronary microvascular obstruction that occurs during reperfusion after acute $\mathrm{MI}$ and is associated with adverse prognosis. Embolization of atherothrombotic debris, platelet and platelet/leukocyte aggregates, vasoconstrictor substances, edema, and physical destruction of the capillary bed all contribute to the no-reflow phenomenon. Minor forms of microvascular obstruction are frequently observed after elective and emergency PCI (Heusch et al., 2013). Coronary microembolization that occurs during early reperfusion may even be initiated by interventional manipulation of the culprit lesion, increasing infarct size. In a pig model of coronary microembolization during early reperfusion, a redistribution of embolizing particles into the infarct border zone is causal for lateral extension of the infarct (Skyschally et al., 2013). Many interventionalists abstain from postconditioning maneuvers by further manipulation of the culprit lesion after primarily successful reperfusion for fear of causing coronary microembolization and additional damage (Heusch, 2012). However, in pigs, ischemic postconditioning is still effective in reducing infarct size even when associated with coronary microembolization, albeit to a lesser extent (Skyschally et al., 2013). Therefore, again it appears wise to perform postconditioning after direct stenting, which prevents coronary microembolization from the culprit lesion (Loubeyre et al., 2002; Heusch, 2012).

Finally, we have to consider the impact of soluble mediators released into the coronary circulation, which not only contribute to microvascular obstruction per se but may also induce cardioprotection. These include notably $\mathrm{TNF} \alpha$ (Skyschally et al., 2007; Kleinbongard et al., 2010; Kleinbongard et al., 2011) and endothelin (Kleinbongard et al., 2013a), but others might also be implicated.
In summary, the coronary circulation is not only the primary origin of myocardial infarction and reperfusion, but also a major confounder of local cardioprotective strategies, i.e., ischemic preconditioning and postconditioning.

\section{Effects of Concomitant Medications Used to Treat Risk Factors and Comorbidities on Cardioprotection: Hidden Cardiotoxicity?}

Here we review recent data on the effect of the most frequently encountered drug therapies for cardiovascular risk factors that may modify cardioprotective mechanisms and thereby modify the efficacy of cardioprotection (Table 3). Very little is known on the possible unwanted effect of medications on the ischemic heart and endogenous cardioprotection, which may result in a latently developing cardiotoxicity (Golomb et al., 2009), and which is now termed as "hidden cardiotoxicity." Hidden cardiotoxicty of a medication may not be seen in the healthy heart but may manifest in only the ischemic heart as an increased tissue injury due to an increased sensitivity to an ischemic challenge or a decreased capability to adapt to an ischemic challenge.

\section{A. Nitrates and Nitrate Tolerance}

Organic nitrates have been used for more than 100 years to prevent and alleviate symptoms of angina pectoris and to reduce blood pressure. However, the main limitation of long-term prophylactic nitrate therapy is the development of vascular nitrate tolerance, which leads to the loss of clinical efficacy (see for review: Csont and Ferdinandy, 2005; Csont, 2010). It has been shown in preclinical studies that the presence of nitrate tolerance aggravates ischemia/reperfusion injury and leads to loss of the cardioprotective effect of preconditioning (see for review Ferdinandy et al., 2007). Since then, little progress has been made in this field. Nevertheless, Fekete et al. (2013) recently showed that the presence of nitrate tolerance inhibits the infarct size limiting effect of postconditioning in rats. Moreover, in a recent human study, Gori et al. (2010) reported that the endothelial preconditioning effect of a single dose of nitroglycerin is lost upon a prolonged exposure to nitroglycerin. Nevertheless, the acute administration of nitrates appears not to interfere with remote conditioning in patients undergoing coronary artery bypass graft surgery (Kleinbongard et al., 2013b).

\section{B. Statins and Antihyperlipidemic Medication}

A number of large clinical trials and meta-analyses has shown that chronic administration of the most frequently used antihyperlipidemic drugs, statins (hydroxymethylglutarate $\mathrm{CoA}$ reductase inhibitors, i.e., inhibitors of the mevalonate pathway), have potent cholesterol-lowering effects and reduce cardiovascular morbidity and mortality (Mills et al., 2011) even in 
TABLE 3

Effect of most common medications of cardiovascular risk factors on ischemia/reperfusion (I/R) injury as well as pre-, post-, and remote conditioning in the majority of the studies

It should be noted that very few data are available so far on the effect of medications on conditioning strategies.

\begin{tabular}{|c|c|c|c|c|}
\hline Medication & I/R Injury & Preconditioning & Postconditioning & Remote Conditioning \\
\hline Nitrate tolerance & $\uparrow$ & $\downarrow$ & $\downarrow$ & N.D. \\
\hline Statins & $\downarrow$ & $\downarrow$ & $\downarrow$ & N.D. \\
\hline \multicolumn{5}{|l|}{ Antidiabetics } \\
\hline KATP inhibitors & $\uparrow$ & $\downarrow$ & $\downarrow$ & $\downarrow$ \\
\hline Others & $\downarrow$ & - & - & N.D. \\
\hline$\beta$-Blockers & $\downarrow$ & $\downarrow-$ & - & N.D. \\
\hline ACE inhibitors & $\downarrow$ & $\uparrow$ & N.D. & N.D. \\
\hline AT1 antagonists & $\downarrow$ & $\uparrow$ & N.D. & N.D. \\
\hline Calcium channel blockers & $\downarrow-$ & - & N.D. & N.D. \\
\hline COX inhibitors & $\uparrow$ & N.D. & N.D. & N.D. \\
\hline
\end{tabular}

N.D., no data available; $\uparrow$, enhance, $\downarrow$, attenuate, - , no effect.

low-risk patients (Mihaylova et al., 2012). However, it seems that statin therapy is less effective in women (Gutierrez et al., 2012). Because the anti-ischemic effect of rosuvastatin, as shown by improved flowmediated dilation after upper arm ischemia, may disappear after chronic treatment in patients (Liuni et al., 2012), acute statin loading therapy has been recommended for PCI recently (Benjo et al., 2014). However, acute statin therapy was found ineffective in a recent clinical trial in low-risk PCI patients (Ludman et al., 2011). The possible mechanisms of the statininduced cardioprotective effect are reviewed elsewhere (Ludman et al., 2009; Mahalwar and Khanna, 2013).

We previously reviewed that statins protect the heart against ischemia/reperfusion injury in preclinical studies but may interfere with the infarct size-limiting effect of preconditioning (see Ferdinandy et al., 2007). However, very little is known on the possible interactions of statins with cardioprotection by conditioning strategies. Kocsis et al. (2008) showed that preconditioning failed to decrease infarct size in rat hearts treated acutely with lovastatin and that postconditioning failed to decrease infarct size after chronic lovastatin treatment. In this study, myocardial levels of coenzyme Q9 and phosphorylation of Akt were decreased due to lovastatin treatment. Moreover, acute, but not chronic lovastatin treatment increased the phosphorylation of p42 ERK. Szucs et al. (2013) recently showed that activation of the mevalonate pathway with farnesol is cardioprotective; thus, one may speculate that inhibition of this pathway by statins may interfere with cardioprotective mechanisms. Accordingly, chronic atorvastatin treatment in diabetic rats with postconditioning failed to show any cardioprotection (Fan et al., 2012). Upregulation by statins of PTEN, which then dephosphorylates PI3K, might contribute to the lack of endogenous cardioprotection after long-term statin treatment (Schulz, 2005). In rabbits, short-term simvastatin treatment and postconditioning were unable to reduce infarct size, but pravastatin decreased infarct size, possibly via eNOS activation (Andreadou et al., 2012). Chronic simvastatin treatment, in contrast to postconditioning, was able to reduce infarct size in both normocholesterolemic and hypercholesterolemic rabbits (Iliodromitis et al., 2010). The effect of statins on remote conditioning has not yet been reported.

In summary, although statins show a potential cardioprotective effect, some statins, depending on the duration of treatment, may interfere with the mechanisms of cardiac adaptation to ischemia/reperfusion stress. This may represent an uncharacterized deleterious effect of statins. Further preclinical and clinical studies are needed to explore the effect of statins on cardioprotection by conditioning strategies. Nevertheless, the development of novel lipid-lowering agents that do not interfere with innate mechanisms of cardiac stress adaptation may further improve the efficacy of lipid-lowering therapy in the prevention of cardiovascular events in hypercholesterolemic patients.

\section{Antidiabetic Therapy}

The treatments used to lower blood glucose levels in diabetic patients may impact on the cardioprotective efficacy of "conditioning" strategies by modulating the underlying intracellular signaling pathways within the heart in two major ways. The antidiabetic therapy may either interfere with the cardioprotective signaling pathway, thereby blocking the "conditioning" strategy, or it may mimic the "conditioning" strategy, thereby inducing cardioprotection (reviewed in Ferdinandy et al., 2007; Ye et al., 2011). Through these effects, there is the potential for antidiabetic therapies to impact on long-term cardiac outcomes in diabetic patients.

In 2008, the US Food and Drug Administration issued requirements that novel antidiabetic drugs must be shown not to increase the risk of cardiovascular events. It is well established that antidiabetic therapy can prevent microvascular complications of diabetes, but whether it can also reduce macrovascular complications and improve major cardiovascular outcomes, such as myocardial infarction, stroke, heart failure, and fatal arrhythmias, is unresolved. 
1. Antidiabetic Therapy and ATP-Sensitive Potassium Channels. The activation of either the sarcolemmal or mitochondrial $\mathrm{K}_{\mathrm{ATP}}$ channel within the cardiomyocyte is a critical step in the signaling pathway underlying both preconditioning and postconditioning (Ferdinandy et al., 2007; Hausenloy, 2013). It is well established in the preclinical literature that some long-acting antidiabetic sulfonylureas (such as tolbutamide and glibenclamide), which act by blocking $\mathrm{K}_{\mathrm{ATP}}$ channel activity in the pancreas to promote insulin release, also interfere with the signaling pathway underlying preconditioning and postconditioning by antagonizing $\mathrm{K}_{\mathrm{ATP}}$ channel activity within the cardiomyocyte (reviewed in Ye et al., 2011). However, some of the newer shorter-acting antidiabetic sulfonylureas, such as gliclazide and glimepiride, do not appear to interfere with cardioprotection (Wu et al., 2007). A recent metaanalysis has confirmed that sulphonylurea use may elevate the risk of cardiovascular disease among patients with diabetes (Phung et al., 2013), although one long-term clinical outcome study failed to report any increase in cardiovascular events in diabetic patients treated with glibenclamide compared with gliclazide (Juurlink et al., 2012).

2. Antidiabetic Therapy with Mixed Actions on Cardioprotection. A number of antidiabetic agents which are used to treat hyperglycemia in diabetic patients are known to confer cardioprotection; many of these appear to act through the activation of the signaling pathways underlying "conditioning" interventions.

Experimental studies have previously established that the antidiabetic agents insulin and metformin can confer cardioprotection in animal MI models (Bhamra et al., 2008; Whittington et al., 2013a). Some of the newer antidiabetic agents have also been reported to limit infarct size in the diabetic and nondiabetic heart, including 1) thiazolidinediones, such as rosiglitazone (Morrison et al., 2011; Palee et al., 2013) and pioglitazone (Ye et al., 2008; 2010a); 2) GLP-1 analogs, such as liraglutide (Noyan-Ashraf et al., 2009) and exenatide (Timmers et al., 2009); and 3) dipeptidyl peptidase-4 inhibitors, such as sitagliptin (Sauve et al., 2010; Ye et al., 2010a; Hausenloy et al., 2013b) and vildagliptin (Hausenloy et al., 2013b). The novel antidiabetic agent mitiglinide (Ogawa et al., 2007) has been found to affect the efficacy of preconditioning or postconditioning in the diabetic heart. However, not all studies have reported cardioprotective effects with these antidiabetic agents (Kristensen et al., 2009; Yin et al., 2011).

A number of clinical studies have either investigated whether antidiabetic agents abolish cardioprotection or have investigated the antidiabetic agent as a therapeutic intervention for limiting MI size. Large longterm clinical outcome studies have examined the effect of the antidiabetic therapy on major cardiovascular events. Hueb et al. (2007) demonstrated in diabetic patients that treatment with repaglinide (a $\mathrm{K}_{\mathrm{ATP}}$ channel antagonist) blocked the ischemic preconditioning effect against exercise-induced myocardial ischemia. Using a similar clinical model, Rahmi et al. (2013) confirmed the negative effect of repaglinide on preconditioning but also found that vildagliptin did not block preconditioning's protection. The protection by remote ischemic preconditioning is abrogated in diabetic CABG patients when treated with sulphonylureas (Kottenberg et al., 2014b).

Administration of the GLP-1 analog exenatide at the time of myocardial reperfusion has been reported to reduce infarct size in STEMI patients treated by primary PCI in two proof-of-concept clinical studies (Lonborg et al., 2012; Woo et al., 2013). The recent IMMEDIATE trial suggests that the beneficial effect of glucose-insulin-potassium therapy in STEMI patients may indeed be operative if administered in the ambulance en route to the primary PCI center while ischemia is ongoing (Selker et al., 2012).

A number of large multicenter long-term clinical outcome studies have reported mixed results in terms of their impact on major ischemia-related cardiovascular outcomes with either better [metformin (Roussel et al., 2010)], worse [rosiglitazone (Nissen and Wolski, 2007)], or no effect [alogliptin (White et al., 2013) and saxagliptin (Scirica et al., 2013)].

In summary, it is important to appreciate that many antidiabetic therapies can either interfere with or mimic the cardioprotective intervention under investigation. It is important to take this into account when designing clinical studies of cardioprotection in diabetic patients.

\section{D. $\beta$-Adrenoceptor Antagonists}

The effect of ischemic preconditioning was abolished in isolated rat hearts after long-term oral treatment with propranolol or nipradilol (Suematsu et al., 2004). In contrast, landilol treatment during ischemic preconditioning in isolated rat hearts ( $\mathrm{Yu}$ et al., 2010) or intravenous atenolol or esmolol, given before ischemia or at reperfusion, did not interfere with protection afforded by ischemic preconditioning in rabbit hearts in vivo (Iliodromitis et al., 2004). However, esmolol abolished the infarct size reduction achieved by fluraneinduced anesthetic preconditioning (Lange et al., 2006). In isolated rat cardiomyocytes, hypoxia or anesthesiainduced 24-hour delayed preconditioning was abolished by metoprolol (Goetzenich et al., 2011). Similarly, anesthesia-induced postconditioning was mediated in part by $\beta$-adrenergic signaling as infarct size reduction was again blocked by esmolol (Lange et al., 2009). Although $\beta$-blockers might thus interfere with the protection afforded by preconditioning and postconditioning in healthy hearts in vivo, carvedilol restored the protection by ischemic preconditioning (Watanabe et al., 2006) or postconditioning (Oikawa et al., 2008) that was 
otherwise lost in hearts with a chronic coronary artery stenosis.

Most recently, a meta-analysis of 15 trials with a total of 1155 patients confirmed that remote ischemic preconditioning significantly reduced postoperative biomarkers of myocardial injury, and this effect was most significant in valve surgery and to a lesser extent in coronary artery bypass graft surgery. One major source of heterogeneity in the protective effect was the use of $\beta$-blockers, suggesting that these drugs might attenuate the cardioprotective effect of remote ischemic preconditioning in patients (Zhou et al., 2013).

Currently, there is no clear answer yet as to whether $\beta$-blockers impact on endogenous cardioprotective signaling. However, the data provided so far highlight the need for further studies and more detailed analyses, given the widespread use of $\beta$-blocker therapy.

\section{E. Angiotensin-Converting Enzyme Inhibitors / Angiotensin II Receptor Type 1 Receptor Antagonists}

ACE inhibitors and angiotensin II receptor type 1 (AT1) receptor antagonists when administered before ischemia and/or reperfusion reduce irreversible myocardial injury (Jalowy et al., 1998; Jancso et al., 2004; Messadi et al., 2010). However, the beneficial effects of ACE inhibitors and/or AT1 receptor antagonists become attenuated when cyclooxygenase-2 (COX-2) inhibitors are simultaneously applied (Jalowy et al., 1998). Both ACE inhibitors and AT1 receptor antagonists lower the threshold to achieve endogenous cardioprotection, especially in hearts with comorbidities (Jaberansari et al., 2001; Ebrahim et al., 2007; Penna et al., 2010).

\section{F. Calcium Channel Blockers}

L-type calcium channel blockers (CCBs) are widely used as antianginal and antihypertensive drugs. Experimentally, they reduce infarct size in almost all animal models of MI, with no apparent chemical class differences between different CCBs (i.e. 1,4-dihydropyridines, verapamil, or diltiazem). However, protection is consistently higher when treatment is started before or during early ischemia, with little benefit seen when the agents are given at reperfusion (Kleinbongard et al., 2012). Contrary to the promising experimental data, clinical trials in acute MI have been disappointing (Opie et al., 2000), probably because the agents need to be given before the onset of ischemia or during the early ischemic phase. Although studies on post- or remote conditioning and CCBs are lacking, a single study in pigs demonstrated no interference of CCBs with cardioprotection by ischemic preconditioning (Wallbridge et al., 1996).

\section{G. Cyclooxygenase Inhibitors}

COX-2 is involved in the protective signaling of preconditioning and postconditioning (Alcindor et al.
2004; Sato et al., 2007; Penna et al., 2008; Tosaka et al., 2011). Blockade of COX-2 can abolish the cardioprotective phenomena, most importantly delayed or second window preconditioning (Przyklenk and Heusch, 2003; Huhn et al., 2009a; Guo et al., 2012). High-dose aspirin, which blocks both COX-1 and COX-2, interferes with endogenous cardioprotection (Ye et al., 2010b). Nevertheless, many patients with an acute MI receive high-dose aspirin $(>300 \mathrm{mg})$ orally or intravenously. Whether patient outcome would be improved with lower doses of aspirin remains to be elucidated, although recently COX-2 inhibition was found to increase mortality in patients post-MI (Olsen et al., 2012). Again, whether such increase in mortality relates to the attenuation of endogenous cardioprotective phenomena or is a consequence of reduced endothelial prostacyclin production on susceptibility to thrombosis remains unanswered.

\section{Conclusions and Future Perspectives}

The discovery of the remarkable cardioprotective effect of innate adaptive responses elicited by different conditioning strategies has fuelled intensive research in the last three decades to find key cellular mechanisms and drug targets for pharmacologic cardioprotection as well as clinically applicable protocols for mechanical cardioprotection. Given the global scale of ischemic heart disease, surprisingly little effort has been made to uncover the cellular mechanisms by which major cardiovascular risk factors (see section IV) and their medications (see section V) may interfere with cardioprotective mechanisms.

Most of the clinical trials with potential cardioprotective drugs have been unsuccessful so far. One of the reasons might be that target validation and preclinical efficacy and safety studies have been performed in juvenile, healthy animals subjected to ischemia/reperfusion injury. Here we have indicated data suggesting that validation of drug targets in comorbid animal models would be essential for successful clinical translation (see section IV.C.2). Furthermore, we have highlighted that routine medications for cardiovascular and other diseases may exert undesirable effects on the ischemic heart and cardioprotective signaling mechanisms, thereby possessing an as yet largely unexplored "hidden cardiotoxicity" (see section V).

Proof-of-principle studies using mechanical conditioning strategies in patients undergoing elective, emergency, or surgical coronary revascularization have successfully translated cardioprotection from animal experiments to human studies, although there are several negative studies (see section III). The reasons for the negative studies can be attributed to the presence of risk factors, comorbidities, and their medications in different patient cohorts. So far, such clinical studies have been completed in small cohorts of selected 
patients under tightly controlled conditions, usually with surrogate rather than clinical outcome endpoints. However, ongoing clinical outcome studies (e.g. CIRCUS, ERICCA, RIPHeart) will further determine whether ischemic conditioning can improve clinical outcomes in different patient cohorts.

The body of evidence we have reviewed here underscores the critical importance of preclinical models and study designs that address cardioprotection specifically in relation to complicating disease states and risk factors. This more sophisticated approach is now an urgent necessity in experimental cardioprotection research to maximize the likelihood of identifying translatable effective approaches to therapeutic protection of the aged or diseased ischemic heart.

\section{Authorship Contributions}

Participated in research design: Ferdinandy, Schulz.

Wrote or contributed to the writing of the manuscript: Ferdinandy, Hausenloy, Heusch, Baxter, Schulz.

\section{References}

Adam T, Sharp S, Opie LH, and Lecour S (2013) Loss of cardioprotection with ischemic preconditioning in aging hearts: role of sirtuin 1 ? J Cardiovasc Pharmacol Ther 18:46-53.

Ahmed RM, Mohamed HA, Ashraf M, Maithili S, Nabil F, Rami R, and Mohamed TI (2013) Effect of remote ischemic preconditioning on serum troponin T level following elective percutaneous coronary intervention. Catheter Cardiovasc Interv $\mathbf{8 2}$ E647-E653.

Ajmani P, Yadav HN, Singh M, and Sharma PL (2011) Possible involvement of caveolin in attenuation of cardioprotective effect of ischemic preconditioning in diabetic rat heart. BMC Cardiovasc Disord 11:43.

Albrecht M, Zitta K, Bein B, Wennemuth G, Broch O, Renner J, Schuett T, Lauer F, Maahs D, Hummitzsch L, et al. (2013) Remote ischemic preconditioning regulates HIF-1 $\alpha$ levels, apoptosis and inflammation in heart tissue of cardiosurgical patients: a pilot experimental study. Basic Res Cardiol 108:314.

Alcindor D, Krolikowski JG, Pagel PS, Warltier DC, and Kersten JR (2004) Cyclooxygenase-2 mediates ischemic, anesthetic, and pharmacologic preconditioning in vivo. Anesthesiology 100:547-554.

Alegria JR, Miller TD, Gibbons RJ, Yi QL, and Yusuf S; Collaborative Organization of RheothRx Evaluation (CORE) Trial Investigators (2007) Infarct size, ejection fraction, and mortality in diabetic patients with acute myocardial infarction treated with thrombolytic therapy. Am Heart $J$ 154:743-750.

Ali N, Rizwi F, Iqbal A, and Rashid A (2010) Induced remote ischemic preconditioning on ischemia-reperfusion injury in patients undergoing coronary artery bypass. J Coll Physicians Surg Pak 20:427-431.

Alkhulaifi AM, Yellon DM, and Pugsley WB (1994) Preconditioning the human heart during aorto-coronary bypass surgery. Eur J Cardiothorac Surg 8:270-275, discussion 276.

Altschuld RA, Hohl CM, Castillo LC, Garleb AA, Starling RC, and Brierley GP (1992) Cyclosporin inhibits mitochondrial calcium efflux in isolated adult rat ventricular cardiomyocytes. Am J Physiol 262:H1699-H1704.

Amr YM and Yassin IM (2010) Cardiac protection during on-pump coronary artery bypass grafting: ischemic versus isoflurane preconditioning. Semin Cardiothorac Vasc Anesth 14:205-211.

Andreadou I, Farmakis D, Prokovas E, Sigala F, Zoga A, Spyridaki K, Papalois A Papapetropoulos A, Anastasiou-Nana M, Kremastinos DT, et al. (2012) Short-term statin administration in hypercholesterolaemic rabbits resistant to postconditioning: effects on infarct size, endothelial nitric oxide synthase, and nitro-oxidative stress. Cardiovasc Res 94:501-509.

Andreadou I, Iliodromitis EK, Rassaf T, Schulz R, Papapetropoulos A, and Ferdinandy $\mathrm{P}$ (2014) The role of gasotransmitters $\mathrm{NO}, \mathrm{H} 2 \mathrm{~S}, \mathrm{CO}$ in myocardial ischemia/ reperfusion injury and cardioprotection by preconditioning, postconditioning, and remote conditioning. $\mathrm{Br} J$ Pharmacol, in press.

Andreka G, Vertesaljai M, Szantho G, Font G, Piroth Z, Fontos G, Juhasz ED, Szekely L, Szelid Z, Turner MS, et al. (2007) Remote ischaemic postconditioning protects the heart during acute myocardial infarction in pigs. Heart 93:749-752.

Argaud L, Gateau-Roesch O, Raisky O, Loufouat J, Robert D, and Ovize M (2005) Postconditioning inhibits mitochondrial permeability transition. Circulation 111:194-197.

Baars T, Konorza T, Kahlert P, Möhlenkamp S, Erbel R, Heusch G, and Kleinbongard $\mathrm{P}$ (2013) Coronary aspirate $\mathrm{TNF} \alpha$ reflects saphenous vein bypass graft restenosis risk in diabetic patients. Cardiovasc Diabetol 12:12.

Babbar L, Mahadevan N, and Balakumar P (2013) Fenofibrate attenuates impaired ischemic preconditioning-mediated cardioprotection in the fructose-fed hypertriglyceridemic rat heart. Naunyn Schmiedebergs Arch Pharmacol 386:319-329.

Babiker FA, van Golde J, Vanagt WY, and Prinzen FW (2012) Pacing postconditioning: impact of pacing algorithm, gender, and diabetes on its myocardial protective effects. J Cardiovasc Transl Res 5:727-734.
Badalzadeh R, Mohammadi M, Najafi M, Ahmadiasl N, Farajnia S, and Ebrahimi H (2012) The additive effects of ischemic postconditioning and cyclosporine-A on nitric oxide activity and functions of diabetic myocardium injured by ischemia/ reperfusion. J Cardiovasc Pharmacol Ther 17:181-189.

Barua A, Standen NB, and Galiñanes M (2011) Modulation of the nitric oxide metabolism overcomes the unresponsiveness of the diabetic human myocardium to protection against ischemic injury. J Surg Res 171:452-456.

Basalay M, Barsukevich V, Mastitskaya S, Mrochek A, Pernow J, Sjöquist PO, Ackland GL, Gourine AV, and Gourine A (2012) Remote ischaemic pre- and delayed postconditioning - similar degree of cardioprotection but distinct mechanisms. Exp Physiol 97:908-917.

Baughman JM, Perocchi F, Girgis HS, Plovanich M, Belcher-Timme CA, Sancak Y, Bao XR, Strittmatter L, Goldberger O, Bogorad RL, et al. (2011) Integrative genomics identifies MCU as an essential component of the mitochondrial calcium uniporter. Nature 476:341-345.

Bautin A, Datsenko S, Tashkhanov D, Gordeev M, Rubinchik V, Kurapeev D, and Galagudza M (2013) Influence of the anesthesia technique on the cardioprotective effects of the remote ischemic preconditioning in the patients undergoing the aortic valve replacement. Heart 99:A40-A41.

Bell R, Beeuwkens R, Botker HE, Davidson S, Downey J, Garcia-Dorado D, Hausenloy DJ, Heusch G, Ibanez B, Kitakaze M, et al. (2012) Trials, tribulations and speculation! Report from the 7th Hatter Cardiovascular Institute Workshop. Basic Res Cardiol 107: 300

Bell RM, Kunuthur SP, Hendry C, Bruce-Hickman D, Davidson S, and Yellon DM (2013) Matrix metalloproteinase inhibition protects CyPD knockout mice independently of RISK/mPTP signalling: a parallel pathway to protection. Basic Res Cardiol 108:331.

Bencsik P, Pálóczi J, Kocsis GF, Pipis J, Belecz I, Varga ZV, Csonka C, Görbe A Csont T, and Ferdinandy P (2014) Moderate inhibition of myocardial matrix metalloproteinase-2 by ilomastat is cardioprotective. Pharmacol Res 80:36-42.

Benjo AM, El-Hayek GE, Messerli F, Dinicolantonio JJ, Hong MK, Aziz EF, Herzog E, and Tamis-Holland JE (2014) High dose statin loading prior to percutaneous coronary intervention decreases cardiovascular events: a meta-analysis of randomized controlled trials. Catheter Cardiovasc Interv, in press.

Bhamra GS, Hausenloy DJ, Davidson SM, Carr RD, Paiva M, Wynne AM, Mocanu MM, and Yellon DM (2008) Metformin protects the ischemic heart by the Aktmediated inhibition of mitochondrial permeability transition pore opening. Basic Res Cardiol 103:274-284.

Bice JS, Burley DS, and Baxter GF (2014) Novel approaches and opportunities for cardioprotective signaling through $3^{\prime}, 5^{\prime}$-cyclic guanosine monophosphate manipulation. J Cardiovasc Pharmacol Ther 19:269-282.

Birincioglu M, Yang X-M, Critz SD, Cohen MV, and Downey JM (1999) S-T segment voltage during sequential coronary occlusions is an unreliable marker of preconditioning. Am J Physiol 277:H2435-H2441.

Birnbaum Y, Hale SL, and Kloner RA (1997) Ischemic preconditioning at a distance: reduction of myocardial infarct size by partial reduction of blood supply combined with rapid stimulation of the gastrocnemius muscle in the rabbit. Circulation 96 $1641-1646$

Boengler K, Buechert A, Heinen Y, Roeskes C, Hilfiker-Kleiner D, Heusch G, and Schulz R (2008a) Cardioprotection by ischemic postconditioning is lost in aged and STAT3-deficient mice. Circ Res 102:131-135.

Boengler K, Heusch G, and Schulz R (2011a) Mitochondria in postconditioning. Antioxid Redox Signal 14:863-880.

Boengler K, Heusch G, and Schulz R (2011b) Nuclear-encoded mitochondrial proteins and their role in cardioprotection. Biochim Biophys Acta 1813:1286-1294.

Boengler K, Hilfiker-Kleiner D, Drexler H, Heusch G, and Schulz R (2008b) The myocardial JAK/STAT pathway: from protection to failure. Pharmacol Ther 120:172-185.

Boengler K, Hilfiker-Kleiner D, Heusch G, and Schulz R (2010) Inhibition of permeability transition pore opening by mitochondrial STAT3 and its role in myocardial ischemia/reperfusion. Basic Res Cardiol 105:771-785.

Boengler K, Ruiz-Meana M, Gent S, Ungefug E, Soetkamp D, Miro-Casas E, Cabestrero A, Fernandez-Sanz C, Semenzato M, Di Lisa F, et al. (2012) Mitochondrial connexin 43 impacts on respiratory complex I activity and mitochondrial oxygen consumption. J Cell Mol Med 16:1649-1655.

Boengler K, Schulz R, and Heusch G (2009) Loss of cardioprotection with ageing. Cardiovasc Res 83:247-261.

Boengler K, Ungefug E, Heusch G, Leybaert L, and Schulz R (2013a) Connexin 43 impacts on mitochondrial potassium uptake. Front Pharmacol 4:73.

Boengler K, Ungefug E, Heusch G, and Schulz R (2013b) The STAT3 inhibitor stattic impairs cardiomyocyte mitochondrial function through increased reactive oxygen species formation. Curr Pharm Des 19:6890-6895.

Bonora M, Bononi A, De Marchi E, Giorgi C, Lebiedzinska M, Marchi S, Patergnani S, Rimessi A, Suski JM, Wojtala A, et al. (2013) Role of the c subunit of the $F_{O}$ ATP synthase in mitochondrial permeability transition. Cell Cycle 12:674-683.

Bopassa JC, Ferrera R, Gateau-Roesch O, Couture-Lepetit E, and Ovize M (2006) PI 3 -kinase regulates the mitochondrial transition pore in controlled reperfusion and postconditioning. Cardiovasc Res 69:178-185.

Bøtker HE, Kharbanda R, Schmidt MR, Bøttcher M, Kaltoft AK, Terkelsen CJ, Munk K, Andersen NH, Hansen TM, Trautner S, et al. (2010) Remote ischaemic conditioning before hospital admission, as a complement to angioplasty, and effect on myocardial salvage in patients with acute myocardial infarction: a randomised trial. Lancet 375:727-734.

Bouhidel O, Pons S, Souktani R, Zini R, Berdeaux A, and Ghaleh B (2008) Myocardial ischemic postconditioning against ischemia-reperfusion is impaired in ob/ob mice. Am J Physiol Heart Circ Physiol 295:H1580-H1586.

Burley DS and Baxter GF (2009) Pharmacological targets revealed by myocardial postconditioning. Curr Opin Pharmacol 9:177-188.

Burley DS, Ferdinandy P, and Baxter GF (2007) Cyclic GMP and protein kinase-G in myocardial ischaemia-reperfusion: opportunities and obstacles for survival signaling. Br J Pharmacol 152:855-869. 
Buyukates M, Kalaycioglu S, Oz E, and Soncul H (2005) Effects of ischemic preconditioning in human heart. J Card Surg 20:241-245.

Byrne CJ, McCafferty K, Kieswich J, Harwood S, Andrikopoulos P, Raftery M, Thiemermann C, and Yaqoob MM (2012) Ischemic conditioning protects the uremic heart in a rodent model of myocardial infarction. Circulation 125:1256-1265.

Canali E, Masci P, Bogaert J, Bucciarelli Ducci C, Francone M, McAlindon E, Carbone I, Lombardi M, Desmet W, Janssens S, et al. (2012) Impact of gender differences on myocardial salvage and post-ischaemic left ventricular remodelling after primary coronary angioplasty: new insights from cardiovascular magnetic resonance. Eur Heart $J$ Cardiovasc Imaging 13:948-953.

Candilio L, Hausenloy DJ, and Yellon DM (2011) Remote ischemic conditioning: a clinical trial's update. J Cardiovasc Pharmacol Ther 16:304-312.

Chang DJ, Chang CH, Kim JS, Hong YW, Lee WK, and Shim YH (2012) Isofluraneinduced post-conditioning in senescent hearts is attenuated by failure to activate reperfusion injury salvage kinase pathway. Acta Anaesthesiol Scand 56:896-903.

Chen Q, Ross T, Hu Y, and Lesnefsky EJ (2012) Blockade of electron transport at the onset of reperfusion decreases cardiac injury in aged hearts by protecting the inner mitochondrial membrane. J Aging Res 2012:753949.

Chen Y and Dorn GW 2nd (2013) PINK1-phosphorylated mitofusin 2 is a Parkin receptor for culling damaged mitochondria. Science 340:471-475.

Cheung MMH, Kharbanda RK, Konstantinov IE, Shimizu M, Frndova H, Li J, Holtby HM, Cox PN, Smallhorn JF, Van Arsdell GS, et al.; First Clinical Application in Humans (2006) Randomized controlled trial of the effects of remote ischemic preconditioning on children undergoing cardiac surgery: first clinical application in humans. J Am Coll Cardiol 47:2277-2282.

Choi YS, Shim JK, Kim JC, Kang KS, Seo YH, Ahn KR, and Kwak YL (2011) Effect of remote ischemic preconditioning on renal dysfunction after complex valvular heart surgery: a randomized controlled trial. J Thorac Cardiovasc Surg 142:148-154.

Clarke SJ, Khaliulin I, Das M, Parker JE, Heesom KJ, and Halestrap AP (2008) Inhibition of mitochondrial permeability transition pore opening by ischemic preconditioning is probably mediated by reduction of oxidative stress rather than mitochondrial protein phosphorylation. Circ Res 102:1082-1090.

Codispoti M, Sundaramoorthi T, Saad RA, Reid A, Sinclair C, and Mankad P (2006) Optimal myocardial protection strategy for coronary artery bypass grafting without cardioplegia: prospective randomised trial. Interact Cardiovasc Thorac Surg $\mathbf{5}$ $217-221$.

Cohen MV, Yang XM, and Downey JM (2007) The $\mathrm{pH}$ hypothesis of postconditioning: staccato reperfusion reintroduces oxygen and perpetuates myocardial acidosis. Circulation 115:1895-1903.

Costa $\mathrm{AD}$ and Garlid $\mathrm{KD}$ (2008) Intramitochondrial signaling: interactions among mitoK $_{\mathrm{ATP}}$, PKCepsilon, ROS, and MPT. Am J Physiol Heart Circ Physiol 295 H874-H882.

Cremer J, Steinhoff G, Karck M, Ahnsell T, Brandt M, Teebken OE, Hollander D, and Haverich A (1997) Ischemic preconditioning prior to myocardial protection with cold blood cardioplegia in coronary surgery. Eur $J$ Cardiothorac Surg 12 $753-758$

Crimi G, Pica S, Raineri C, Bramucci E, De Ferrari GM, Klersy C, Ferlini M, Marinoni B, Repetto A, Romeo M, et al. (2013) Remote ischemic post-conditioning of the lower limb during primary percutaneous coronary intervention safely reduces enzymatic infarct size in anterior myocardial infarction: a randomized controlled trial. JACC Cardiovasc Interv 6:1055-1063.

Csonka C, Kupai K, Bencsik P, Görbe A, Pálóczi J, Zvara A, Puskás LG, Csont T, and Ferdinandy P (2014) Cholesterol-enriched diet inhibits cardioprotection by ATP-sensitive $\mathrm{K}+$ channel activators cromakalim and diazoxide. Am J Physiol Heart Circ Physiol 306:H405-H413.

Csont T (2010) Nitroglycerin-induced preconditioning: interaction with nitrate tolerance. Am J Physiol Heart Circ Physiol 298:H308-H309.

Csont T and Ferdinandy P (2005) Cardioprotective effects of glyceryl trinitrate: beyond vascular nitrate tolerance. Pharmacol Ther 105:57-68.

Dai W, Simkhovich BZ, and Kloner RA (2009) Ischemic preconditioning maintains cardioprotection in aging normotensive and spontaneously hypertensive rats. Exp Gerontol 44:344-349.

Darling CE, Solari PB, Smith CS, Furman MI, and Przyklenk K (2007) 'Postconditioning' the human heart: multiple balloon inflations during primary angioplasty may confer cardioprotection. Basic Res Cardiol 102:274-278.

Davidson SM, Selvaraj P, He D, Boi-Doku C, Yellon RL, Vicencio JM, and Yellon DM (2013) Remote ischaemic preconditioning involves signalling through the SDF-1 $\alpha$ CXCR4 signalling axis. Basic Res Cardiol 108:377.

Davies WR, Brown AJ, Watson W, McCormick LM, West NE, Dutka DP, and Hoole SP (2013) Remote ischemic preconditioning improves outcome at 6 years after elective percutaneous coronary intervention: the CRISP stent trial long-term follow-up. Circ Cardiovasc Interv 6:246-251.

de Brito OM and Scorrano L (2008a) Mitofusin 2 tethers endoplasmic reticulum to mitochondria. Nature 456:605-610.

de Brito OM and Scorrano L (2008b) Mitofusin 2: a mitochondria-shaping protein with signaling roles beyond fusion. Antioxid Redox Signal 10:621-633.

De Paulis D, Chiari P, Teixeira G, Couture-Lepetit E, Abrial M, Argaud L, Gharib A, and Ovize M (2013) Cyclosporine A at reperfusion fails to reduce infarct size in the in vivo rat heart. Basic Res Cardiol 108:379.

De Stefani D, Raffaello A, Teardo E, Szabò I, and Rizzuto R (2011) A forty-kilodalton protein of the inner membrane is the mitochondrial calcium uniporter. Nature $\mathbf{4 7 6}$ $336-340$

Disatnik MH, Ferreira JC, Campos JC, Gomes KS, Dourado PM, Qi X, and MochlyRosen D (2013) Acute inhibition of excessive mitochondrial fission after myocardial infarction prevents long-term cardiac dysfunction. J Am Heart Assoc 2:e000461.

Donato M, D’Annunzio V, Berg G, Gonzalez G, Schreier L, Morales C, Wikinski RL, and Gelpi RJ (2007) Ischemic postconditioning reduces infarct size by activation of $\mathrm{A}_{1}$ receptors and $\left.\mathrm{K}_{\text {ATP }}^{+(}\right)$channels in both normal and hypercholesterolemic rabbits. J Cardiovasc Pharmacol 49:287-292.
Dormán G, Cseh S, Hajdú I, Barna L, Kónya D, Kupai K, Kovács L, and Ferdinandy P (2010) Matrix metalloproteinase inhibitors: a critical appraisal of design principles and proposed therapeutic utility. Drugs 70:949-964.

Drenger B, Ostrovsky IA, Barak M, Nechemia-Arbely Y, Ziv E, and Axelrod JH (2011) Diabetes blockade of sevoflurane postconditioning is not restored by insulin in the rat heart: phosphorylated signal transducer and activator of transcription 3- and phosphatidylinositol 3-kinase-mediated inhibition. Anesthesiology 114:1364-1372. Durdu S, Sirlak M, Cetintas D, Inan MB, Eryılmaz S, Ozcinar E, Yazicioglu L, Elhan AH, Akar AR, and Uysalel A (2012) The efficacies of modified mechanical post conditioning on myocardial protection for patients undergoing coronary artery bypass grafting. J Cardiothorac Surg 7:73.

Dwyer NB, Mikami Y, Hilland D, Aljizeeri A, Friedrich MG, Traboulsi M, and Anderson TJ (2013) No cardioprotective benefit of ischemic postconditioning in patients with ST-segment elevation myocardial infarction. J Interv Cardiol 26 $482-490$

Ebrahim Z, Yellon DM, and Baxter GF (2007) Ischemic preconditioning is lost in aging hypertensive rat heart: independent effects of aging and longstanding hypertension. Exp Gerontol 42:807-814

Elrod JW, Wong R, Mishra S, Vagnozzi RJ, Sakthievel B, Goonasekera SA, Karch J, Gabel S, Farber J, Force T, et al. (2010) Cyclophilin D controls mitochondrial poredependent $\mathrm{Ca}^{2+}$ ) exchange, metabolic flexibility, and propensity for heart failure in mice. J Clin Invest 120:3680-3687.

Elżbieciak M, Wita K, Grabka M, Chmurawa J, Doruchowska A, Turski M, Filipecki A, Wybraniec M, and Mizia-Stec K (2013) Effect of postconditioning on infarction size, adverse left ventricular remodeling, and improvement in left ventricular systolic function in patients with first anterior ST-segment elevation myocardia infarction. Pol Arch Med Wewn 123:268-276.

Engbersen R, Riksen NP, Mol MJ, Bravenboer B, Boerman OC, Meijer P, Oyen WJ, Tack C, Rongen GA, and Smits P (2012) Improved resistance to ischemia and reperfusion, but impaired protection by ischemic preconditioning in patients with type 1 diabetes mellitus: a pilot study. Cardiovasc Diabetol 11:124.

Evgenov OV, Pacher P, Schmidt PM, Haskó G, Schmidt HH, and Stasch JP (2006) NO-independent stimulators and activators of soluble guanylate cyclase: discovery and therapeutic potential. Nat Rev Drug Discov 5:755-768.

Fan Y, Yang S, Zhang X, Cao Y, and Huang Y (2012) Comparison of cardioprotective efficacy resulting from a combination of atorvastatin and ischaemic postconditioning in diabetic and non-diabetic rats. Clin Exp Pharmacol Physiol 39: 938-943.

Fekete V, Murlasits Z, Aypar E, Bencsik P, Sárközy M, Szénási G, Ferdinandy P, and Csont T (2013) Myocardial postconditioning is lost in vascular nitrate tolerance. J Cardiovasc Pharmacol 62:298-303.

Ferdinandy P (2003) Myocardial ischaemia/reperfusion injury and preconditioning: effects of hypercholesterolaemia/hyperlipidaemia. Br J Pharmacol 138:283-285.

Ferdinandy P, Schulz R, and Baxter GF (2007) Interaction of cardiovascular risk factors with myocardial ischemia/reperfusion injury, preconditioning, and postconditioning. Pharmacol Rev 59:418-458.

Ferdinandy P, Szilvassy Z, and Baxter GF (1998) Adaptation to myocardial stress in disease states: is preconditioning a healthy heart phenomenon? Trends Pharmacol Sci 19:223-229.

Ferdinandy P, Szilvássy Z, Horváth LI, Csont T, Csonka C, Nagy E, Szentgyörgyi R, Nagy I, Koltai M, and Dux L (1997) Loss of pacing-induced preconditioning in rat hearts: role of nitric oxide and cholesterol-enriched diet. J Mol Cell Cardiol 29: 3321-3333.

Freixa X, Bellera N, Ortiz-Pérez JT, Jiménez M, Paré C, Bosch X, De Caralt TM Betriu A, and Masotti M (2012) Ischaemic postconditioning revisited: lack of effects on infarct size following primary percutaneous coronary intervention. Eur Heart $J$ 33:103-112.

Fujita M, Asanuma H, Hirata A, Wakeno M, Takahama H, Sasaki H, Kim J, Takashima S, Tsukamoto O, Minamino T, et al. (2007) Prolonged transient acidosis during early reperfusion contributes to the cardioprotective effects of postconditioning. Am J Physiol Heart Circ Physiol 292:H2004-H2008.

Fullmer TM, Pei S, Zhu Y, Sloan C, Manzanares R, Henrie B, Pires KM, Cox JE, Abel $\mathrm{ED}$, and Boudina S (2013) Insulin suppresses ischemic preconditioning-mediated cardioprotection through Akt-dependent mechanisms. J Mol Cell Cardiol 64: $20-29$

Garcia S, Henry TD, Wang YL, Chavez IJ, Pedersen WR, Lesser JR, Shroff GR, Moore L, and Traverse JH (2011) Long-term follow-up of patients undergoing postconditioning during ST-elevation myocardial infarction. J Cardiovasc Transl Res 4:92-98.

Garcia-Dorado D, Agulló L, Sartorio CL, and Ruiz-Meana M (2009) Myocardial protection against reperfusion injury: the cGMP pathway. Thromb Haemost 101: $635-642$

Ghosh S and Galiñanes M (2003) Protection of the human heart with ischemic preconditioning during cardiac surgery: role of cardiopulmonary bypass. $J$ Thorac Cardiovasc Surg 126:133-142.

Giorgio V, von Stockum S, Antoniel M, Fabbro A, Fogolari F, Forte M, Glick GD, Petronilli V, Zoratti M, Szabó I, et al. (2013) Dimers of mitochondrial ATP synthase form the permeability transition pore. Proc Natl Acad Sci USA 110:5887-5892.

Giricz Z, Görbe A, Pipis J, Burley DS, Ferdinandy P, and Baxter GF (2009) Hyperlipidaemia induced by a high-cholesterol diet leads to the deterioration of guanosine$3^{\prime}, 5^{\prime}$-cyclic monophosphate/protein kinase G-dependent cardioprotection in rats. $\mathrm{Br}$ J Pharmacol 158:1495-1502.

Giricz Z, Lalu MM, Csonka C, Bencsik P, Schulz R, and Ferdinandy P (2006) Hyperlipidemia attenuates the infarct size-limiting effect of ischemic preconditioning: role of matrix metalloproteinase-2 inhibition. J Pharmacol Exp Ther 316:154-161. Giricz Z, Mentzer RM Jr, and Gottlieb RA (2012) Autophagy, myocardial protection, and the metabolic syndrome. J Cardiovasc Pharmacol 60:125-132.

Giricz Z, Varga ZV, Baranyai T, Sipos P, Pálóczi K, Kittel Á, Buzás EI, and Ferdinandy P (2014) Cardioprotection by remote ischemic preconditioning of the rat heart is mediated by extracellular vesicles. J Mol Cell Cardiol 68:75-78. 
Goetzenich A, Roehl AB, Moza A, Srecec D, Beyer C, Arnold S, and Hein M (2011) The effects of metoprolol on hypoxia- and isoflurane-induced cardiac late-phase preconditioning. Acta Anaesthesiol Scand 55:862-869.

Golomb E, Nyska A, and Schwalb H (2009) Occult cardiotoxicity-toxic effects on cardiac ischemic tolerance. Toxicol Pathol 37:572-593.

Görbe A, Giricz Z, Szunyog A, Csont T, Burley DS, Baxter GF, and Ferdinandy P (2010) Role of cGMP-PKG signaling in the protection of neonatal rat cardiac myocytes subjected to simulated ischemia/reoxygenation. Basic Res Cardiol 105: $643-650$

Görbe A, Varga ZV, Kupai K, Bencsik P, Kocsis GF, Csont T, Boengler K, Schulz R, and Ferdinandy P (2011) Cholesterol diet leads to attenuation of ischemic preconditioning-induced cardiac protection: the role of connexin 43. Am J Physiol Heart Circ Physiol 300:H1907-H1913.

Gori T, Dragoni S, Di Stolfo G, Sicuro S, Liuni A, Luca MC, Thomas G, Oelze M, Daiber A, and Parker JD (2010) Tolerance to nitroglycerin-induced preconditioning of the endothelium: a human in vivo study. Am J Physiol Heart Circ Physiol 298: H340-H345.

Gu W, Kehl F, Krolikowski JG, Pagel PS, Warltier DC, and Kersten JR (2008) Simvastatin restores ischemic preconditioning in the presence of hyperglycemia through a nitric oxide-mediated mechanism. Anesthesiology 108:634-642.

Günaydin B, Cakici I, Soncul H, Kalaycioglu S, Cevik C, Sancak B, Kanzik I, and Karadenizli Y (2000) Does remote organ ischaemia trigger cardiac preconditioning during coronary artery surgery? Pharmacol Res 41:493-496.

Guo Y, Tukaye DN, Wu WJ, Zhu X, Book M, Tan W, Jones SP, Rokosh G, Narumiya $\mathrm{S}$, Li Q, et al. (2012) The COX-2/PGI2 receptor axis plays an obligatory role in mediating the cardioprotection conferred by the late phase of ischemic preconditioning. PLoS ONE 7:e41178.

Gurel E, Ustunova S, Kapucu A, Yilmazer N, Eerbeek O, Nederlof R, Hollmann MW, Demirci-Tansel C, and Zuurbier CJ (2013) Hexokinase cellular trafficking in ischemia-reperfusion and ischemic preconditioning is altered in type I diabetic heart. Mol Biol Rep 40:4153-4160.

Gutierrez J, Ramirez G, Rundek T, and Sacco RL (2012) Statin therapy in the prevention of recurrent cardiovascular events: a sex-based meta-analysis. Arch Intern Med 172:909-919.

Hahn JY, Song YB, Kim EK, Yu CW, Bae JW, Chung WY, Choi SH, Choi JH, Bae JH, An KJ, et al. (2013) Ischemic postconditioning during primary percutaneous coronary intervention: the effects of postconditioning on myocardial reperfusion in patients with ST-segment elevation myocardial infarction (POST) randomized trial Circulation 128:1889-1896.

Halestrap AP, Clarke SJ, and Javadov SA (2004) Ischemic postconditioning during primary percutaneous coronary intervention: the effects of postconditioning on myocardial reperfusion in patients with ST-segment elevation myocardial infarction (POST) randomized trial. Cardiovasc Res 61:372-385.

Hausenloy DJ (2013) Cardioprotection techniques: preconditioning, postconditioning and remote conditioning (basic science). Curr Pharm Des 19:4544-4563.

Hausenloy DJ and Yellon DM (2007) Reperfusion injury salvage kinase signalling: taking a RISK for cardioprotection. Heart Fail Rev 12.217-234

Hausenloy DJ and Yellon DM (2008) Remote ischaemic preconditioning: underlying mechanisms and clinical application. Cardiovasc Res 79:377-386.

Hausenloy DJ, Baxter G, Bell R, Bøtker HE, Davidson SM, Downey J, Heusch G, Kitakaze M, Lecour S, Mentzer R, et al. (2010) Translating novel strategies for cardioprotection: the Hatter Workshop Recommendations. Basic Res Cardiol 105 $677-686$.

Hausenloy DJ, Candilio L, Laing C, Kunst G, Pepper J, Kolvekar S, Evans R, Robertson S, Knight R, Ariti C, et al ; ERICCA Trial Investigators (2012) Effect of remote ischemic preconditioning on clinical outcomes in patients undergoing coronary artery bypass graft surgery (ERICCA): rationale and study design of a multicentre randomized double-blinded controlled clinical trial. Clin Res Cardiol 101: 339-348.

Hausenloy DJ, Bøtker HE, Condorelli G, Ferdinandy P, Garcia-Dorado D, Heusch G, Lecour S, van Laake LW, Madonna R, and Ruiz-Meana M, et al. (2013a) Translating cardioprotection for patient benefit: position paper from the Working Group of Cellular Biology of the Heart of the European Society of Cardiology. Cardiovasc Res 98:7-27.

Hausenloy DJ, Lecour S, and Yellon DM (2011) Reperfusion injury salvage kinase and survivor activating factor enhancement prosurvival signaling pathways in ischemic postconditioning: two sides of the same coin. Antioxid Redox Signal 14: 893-907.

Hausenloy DJ, Mwamure PK, Venugopal V, Harris J, Barnard M, Grundy E, Ashley E, Vichare S, Di Salvo C, Kolvekar S, et al. (2007) Effect of remote ischaemic preconditioning on myocardial injury in patients undergoing coronary artery bypass graft surgery: a randomised controlled trial. Lancet 370:575-579.

Hausenloy DJ, Ong SB, and Yellon DM (2009) The mitochondrial permeability transition pore as a target for preconditioning and postconditioning. Basic Res Cardiol 104:189-202.

Hausenloy DJ, Tsang A, and Yellon DM (2005) The reperfusion injury salvage kinase pathway: a common target for both ischemic preconditioning and postconditioning. Trends Cardiovasc Med 15:69-75.

Hausenloy DJ, Whittington HJ, Wynne AM, Begum SS, Theodorou L, Riksen N, Mocanu MM, and Yellon DM (2013b) Dipeptidyl peptidase-4 inhibitors and GLP-1 reduce myocardial infarct size in a glucose-dependent manner. Cardiovasc Diabetol 12:154

Hausenloy DJ, Wynne AM, Mocanu MM, and Yellon DM (2013c) Glimepiride treatment facilitates ischemic preconditioning in the diabetic heart. $J$ Cardiovasc Pharmacol Ther 18:263-269.

Heinen A, Huhn R, Smeele KM, Zuurbier CJ, Schlack W, Preckel B, Weber NC, and Hollmann MW (2008) Helium-induced preconditioning in young and old rat heart: impact of mitochondrial $\left.\mathrm{Ca}^{2+}\right)$-sensitive potassium channel activation. $\mathrm{An}$ esthesiology 109:830-836.
Heinzel FR, Luo Y, Li X, Boengler K, Buechert A, García-Dorado D, Di Lisa F, Schulz $\mathrm{R}$, and Heusch $\mathrm{G}$ (2005) Impairment of diazoxide-induced formation of reactive oxygen species and loss of cardioprotection in connexin 43 deficient mice. Circ Res 97:583-586.

Heusch G (2001) Nitroglycerin and delayed preconditioning in humans: yet another new mechanism for an old drug? Circulation 103:2876-2878.

Heusch G (2004) Postconditioning: old wine in a new bottle? J Am Coll Cardiol 44: 1111-1112.

Heusch G (2012) Reduction of infarct size by ischaemic post-conditioning in humans: fact or fiction? Eur Heart $J$ 33:13-15.

Heusch G (2013) Cardioprotection: chances and challenges of its translation to the clinic. Lancet 381:166-175.

Heusch G, Boengler K, and Schulz R (2008) Cardioprotection: nitric oxide, protein kinases, and mitochondria. Circulation 118:1915-1919.

Heusch G, Buechert A, Feldhaus S, and Schulz R (2006) No loss of cardioprotection by postconditioning in connexin 43-deficient mice. Basic Res Cardiol 101:354-356.

Heusch G, Kleinbongard P, and Skyschally A (2013) Myocardial infarction and coronary microvascular obstruction: an intimate, but complicated relationship. Basic Res Cardiol 108:380.

Heusch G, Kleinbongard P, Böse D, Levkau B, Haude M, Schulz R, and Erbel R (2009) Coronary microembolization: from bedside to bench and back to bedside. Circulation 120:1822-1836.

Heusch G, Kleinbongard P, Skyschally A, Levkau B, Schulz R, and Erbel R (2012a) The coronary circulation in cardioprotection: more than just one confounder. Cardiovasc Res 94:237-245.

Heusch G, Libby P, Gersh B, Yellon D, Böhm M, Lopaschuk G, and Opie L (2014) Cardiovascular remodelling in coronary artery disease and heart failure. Lancet 383:1933-1943.

Heusch G, Musiolik J, Gedik N, and Skyschally A (2011) Mitochondrial STAT3 activation and cardioprotection by ischemic postconditioning in pigs with regional myocardial ischemia/reperfusion. Circ Res 109:1302-1308.

Heusch G, Musiolik J, Kottenberg E, Peters J, Jakob H, and Thielmann M (2012b) STAT5 activation and cardioprotection by remote ischemic preconditioning in humans: short communication. Circ Res 110:111-115.

Heusch P, Skyschally A, Leineweber K, Haude M, Erbel R, and Heusch G (2007) The interaction of coronary microembolization and ischemic preconditioning: a third window of cardioprotection through TNF-alpha. Arch Med Sci 3:83-92.

Hochhauser E, Leshem D, Kaminski O, Cheporko Y, Vidne BA, and Shainberg A (2007) The protective effect of prior ischemia reperfusion adenosine A1 or A3 receptor activation in the normal and hypertrophied heart. Interact Cardiovasc Thorac Surg 6:363-368.

Hong DM, Jeon Y, Lee CS, Kim HJ, Lee JM, Bahk JH, Kim KB, and Hwang HY (2012) Effects of remote ischemic preconditioning with postconditioning in patients undergoing off-pump coronary artery bypass surgery—randomized controlled trial. Circ J 76:884-890.

Hong DM, Lee EH, Kim HJ, Min JJ, Chin JH, Choi DK, Bahk JH, Sim JY, Choi IC, and Jeon Y (2014) Does remote ischaemic preconditioning with postconditioning improve clinical outcomes of patients undergoing cardiac surgery? Remote Ischaemic Preconditioning with Postconditioning Outcome Trial. Eur Heart J 35: 176-183.

Hong DM, Mint JJ, Kim JH, Sohn IS, Lim TW, Lim YJ, Bahk JH, and Jeon Y (2010) The effect of remote ischaemic preconditioning on myocardial injury in patients undergoing off-pump coronary artery bypass graft surgery. Anaesth Intensive Care 38:924-929.

Hoole SP, Heck PM, Sharples L, Khan SN, Duehmke R, Densem CG, Clarke SC, Shapiro LM, Schofield PM, O'Sullivan M, et al. (2009) Cardiac Remote Ischemic Preconditioning in Coronary Stenting (CRISP Stent) Study: a prospective, randomized control trial. Circulation 119:820-827.

Hotta H, Miura T, Miki T, Togashi N, Maeda T, Kim SJ, Tanno M, Yano T, Kuno A, Itoh T, et al. (2010) Angiotensin II type 1 receptor-mediated upregulation of calcineurin activity underlies impairment of cardioprotective signaling in diabetic hearts. Circ Res 106:129-132.

Huang C, Andres AM, Ratliff EP, Hernandez G, Lee P, and Gottlieb RA (2011) Preconditioning involves selective mitophagy mediated by Parkin and p62/ SQSTM1. PLoS ONE 6:e20975.

Hueb W, Uchida AH, Gersh BJ, Betti RT, Lopes N, Moffa PJ, Ferreira BM, Ramires JA, and Wajchenberg BL (2007) Effect of a hypoglycemic agent on ischemic preconditioning in patients with type 2 diabetes and stable angina pectoris. Coron Artery Dis 18:55-59.

Huhn R, Heinen A, Hollmann MW, Schlack W, Preckel B, and Weber NC (2010) Cyclosporine A administered during reperfusion fails to restore cardioprotection in prediabetic Zucker obese rats in vivo. Nutr Metab Cardiovasc Dis 20:706-712.

Huhn R, Heinen A, Weber NC, Hieber S, Hollmann MW, Schlack W, and Preckel B (2009a) Helium-induced late preconditioning in the rat heart in vivo. $\mathrm{Br} J$ Anaesth 102:614-619.

Huhn R, Heinen A, Weber NC, Kerindongo RP, Oei GT, Hollmann MW, Schlack W, and Preckel B (2009b) Helium-induced early preconditioning and postconditioning are abolished in obese Zucker rats in vivo. J Pharmacol Exp Ther 329:600-607.

Huhn R, Weber NC, Preckel B, Schlack W, Bauer I, Hollmann MW, and Heinen A (2012) Age-related loss of cardiac preconditioning: impact of protein kinase A. Exp Gerontol 47:116-121

Hunter JC and Korzick DH (2005) Age- and sex-dependent alterations in protein kinase $\mathrm{C}(\mathrm{PKC})$ and extracellular regulated kinase 1/2 (ERK1/2) in rat myocardium. Mech Ageing Dev 126:535-550.

Ibanez B, Macaya C, Sánchez-Brunete V, Pizarro G, Fernández-Friera L, Mateos A, Fernández-Ortiz A, García-Ruiz JM, García-Álvarez A, Iñiguez A, et al. (2013) Effect of early metoprolol on infarct size in ST-segment-elevation myocardial infarction patients undergoing primary percutaneous coronary intervention: the Effect of Metoprolol in Cardioprotection During an Acute Myocardial Infarction (METOCARD-CNIC) trial. Circulation 128:1495-1503. 
Iliodromitis EK, Andreadou I, Prokovas E, Zoga A, Farmakis D, Fotopoulou T, Ioannidis K, Paraskevaidis IA, and Kremastinos DT (2010) Simvastatin in contrast to postconditioning reduces infarct size in hyperlipidemic rabbits: possible role of oxidative/nitrosative stress attenuation. Basic Res Cardiol 105:193-203.

Iliodromitis EK, Kyrzopoulos S, Paraskevaidis IA, Kolocassides KG, Adamopoulos S, Karavolias G, and Kremastinos DT (2006) Increased C reactive protein and cardiac enzyme levels after coronary stent implantation. Is there protection by remote ischaemic preconditioning? Heart 92:1821-1826.

Iliodromitis EK, Tasouli A, Andreadou I, Bofilis E, Zoga A, Cokkinos P, and Kremastinos DT (2004) Intravenous atenolol and esmolol maintain the protective effect of ischemic preconditioning in vivo. Eur J Pharmacol 499:163-169.

Illes RW and Swoyer KD (1998) Prospective, randomized clinical study of ischemic preconditioning as an adjunct to intermittent cold blood cardioplegia. Ann Thorac Surg 65:748-752, discussion 752-753.

Jaberansari MT, Baxter GF, Muller CA, Latouf SE, Röth E, Opie LH, and Yellon DM (2001) Angiotensin-converting enzyme inhibition enhances a subthreshold stimulus to elicit delayed preconditioning in pig myocardium. J Am Coll Cardiol 37: 1996-2001.

Jalowy A, Schulz R, Dörge H, Behrends M, and Heusch G (1998) Infarct size reduction by AT1-receptor blockade through a signal cascade of AT2-receptor activation, bradykinin and prostaglandins in pigs. J Am Coll Cardiol 32:1787-1796.

Jancso G, Jaberansari M, Gasz B, Szanto Z, Cserepes B, and Röth E (2004) Bradykinin and angiotensin-converting enzyme inhibition in cardioprotection. Exp Clin Cardiol 9:21-25.

Jang Y, Xi J, Wang H, Mueller RA, Norfleet EA, and Xu Z (2008) Postconditioning prevents reperfusion injury by activating delta-opioid receptors. Anesthesiology 108:243-250

Jebeli M, Esmaili HR, Mandegar MH, Rasouli MR, Eghtesadi-Araghi P, Mohammadzadeh R, Darehzereshki A, and Dianat S (2010) Evaluation of the effects of ischemic preconditioning with a short reperfusion phase on patients undergoing a coronary artery bypass graft. Ann Thorac Cardiovasc Surg 16:248-252.

Jenkins DP, Pugsley WB, Alkhulaifi AM, Kemp M, Hooper J, and Yellon DM (1997) Ischaemic preconditioning reduces troponin $\mathrm{T}$ release in patients undergoing coronary artery bypass surgery. Heart 77:314-318.

Jensen RV, Støttrup NB, Kristiansen SB, and Bøtker HE (2012) Release of a humoral circulating cardioprotective factor by remote ischemic preconditioning is dependent on preserved neural pathways in diabetic patients. Basic Res Cardiol 107:285

Jensen RV, Zachara NE, Nielsen PH, Kimose HH, Kristiansen SB, and Bøtker HE (2013) Impact of O-GlcNAc on cardioprotection by remote ischaemic preconditioning in non-diabetic and diabetic patients. Cardiovasc Res 97:369-378.

Ji B, Liu M, Liu J, Wang G, Feng W, Lu F, and Shengshou H (2007) Evaluation by cardiac troponin I: the effect of ischemic preconditioning as an adjunct to intermittent blood cardioplegia on coronary artery bypass grafting. J Card Surg 22 394-400.

Ji Q, Mei Y, Wang X, Feng J, Wusha D, Cai J, and Zhou Y (2011) Effect of ischemic postconditioning in correction of tetralogy of Fallot. Int Heart $J$ 52:312-317.

Jin ZQ, Karliner JS, and Vessey DA (2008) Ischaemic postconditioning protects isolated mouse hearts against ischaemia/reperfusion injury via sphingosine kinase isoform-1 activation. Cardiovasc Res 79:134-140.

Jones BO, Pepe S, Sheeran FL, Donath S, Hardy P, Shekerdemian L, Penny DJ, McKenzie I, Horton S, Brizard CP, et al. (2013) Remote ischemic preconditioning in cyanosed neonates undergoing cardiopulmonary bypass: a randomized controlled trial. J Thorac Cardiovasc Surg 146:1334-1340.

Juhaszova M, Zorov DB, Kim SH, Pepe S, Fu Q, Fishbein KW, Ziman BD, Wang S, Ytrehus K, Antos CL, et al. (2004) Glycogen synthase kinase-3beta mediates convergence of protection signaling to inhibit the mitochondrial permeability transition pore. J Clin Invest 113:1535-1549.

Juurlink DN, Gomes T, Shah BR, and Mamdani MM (2012) Adverse cardiovascular events during treatment with glyburide (glibenclamide) or gliclazide in a high-risk population. Diabet Med 29:1524-1528.

Kaczmarek A, Vandenabeele P, and Krysko DV (2013) Necroptosis: the release of damage-associated molecular patterns and its physiological relevance. Immunity 38:209-223.

Kamga Pride C, Mo L, Quesnelle K, Dagda RK, Murillo D, Geary L, Corey C, Portella R, Zharikov S, St Croix C, et al. (2014) Nitrite activates protein kinase A in normoxia to mediate mitochondrial fusion and tolerance to ischaemia/reperfusion. Cardiovasc Res 101:57-68.

Karuppasamy P, Chaubey S, Dew T, Musto R, Sherwood R, Desai J, John L, Shah AM, Marber MS, and Kunst G (2011) Remote intermittent ischemia before coronary artery bypass graft surgery: a strategy to reduce injury and inflammation? Basic Res Cardiol 106:511-519.

Kaukoranta PK, Lepojärvi MP, Ylitalo KV, Kiviluoma KT, and Peuhkurinen KJ (1997) Normothermic retrograde blood cardioplegia with or without preceding ischemic preconditioning. Ann Thorac Surg 63:1268-1274.

Kharbanda RK, Mortensen UM, White PA, Kristiansen SB, Schmidt MR, Hoschtitzky JA, Vogel M, Sorensen K, Redington AN, and MacAllister R (2002) Transient limb ischemia induces remote ischemic preconditioning in vivo. Circulation 106: 2881-2883.

Kim HS, Cho JE, Hwang KC, Shim YH, Lee JH, and Kwak YL (2010) Diabetes mellitus mitigates cardioprotective effects of remifentanil preconditioning in ischemia-reperfused rat heart in association with anti-apoptotic pathways of survival. Eur J Pharmacol 628:132-139.

Kin H, Zatta AJ, Lofye MT, Amerson BS, Halkos ME, Kerendi F, Zhao ZQ, Guyton RA, Headrick JP, and Vinten-Johansen J (2005) Postconditioning reduces infarct size via adenosine receptor activation by endogenous adenosine. Cardiovasc Res 67:124-133.

King N, Al Shaama M, and Suleiman MS (2012) Propofol improves recovery of the isolated working hypertrophic heart from ischaemia-reperfusion. Pflugers Arch 464:513-522.
Kitakaze M, Asakura M, Kim J, Shintani Y, Asanuma H, Hamasaki T, Seguchi O, Myoishi M, Minamino T, Ohara T, et al.; J-WIND investigators (2007) Human atrial natriuretic peptide and nicorandil as adjuncts to reperfusion treatment for acute myocardial infarction (J-WIND): two randomised trials. Lancet 370: 1483-1493.

Kleinbongard P, Baars T, and Heusch G (2012) Calcium antagonists in myocardial ischemia/reperfusion-update 2012. Wien Med Wochenschr 162:302-310.

Kleinbongard P, Baars T, Möhlenkamp S, Kahlert P, Erbel R, and Heusch G (2013a) Aspirate from human stented native coronary arteries vs. saphenous vein grafts: more endothelin but less particulate debris. Am J Physiol Heart Circ Physiol 305: H1222-H1229.

Kleinbongard P, Böse D, Baars T, Möhlenkamp S, Konorza T, Schöner S, ElterSchulz M, Eggebrecht H, Degen H, Haude M, et al. (2011) Vasoconstrictor potential of coronary aspirate from patients undergoing stenting of saphenous vein aortocoronary bypass grafts and its pharmacological attenuation. Circ Res 108:344-352.

Kleinbongard P, Heusch G, and Schulz R (2010) TNFalpha in atherosclerosis, myocardial ischemia/reperfusion and heart failure. Pharmacol Ther 127:295-314.

Kleinbongard P, Thielmann M, Jakob H, Peters J, Heusch G, and Kottenberg E (2013b) Nitroglycerin does not interfere with protection by remote ischemic preconditioning in patients with surgical coronary revascularization under isoflurane anesthesia. Cardiovasc Drugs Ther 27:359-361.

Kocsis GF, Pipis J, Fekete V, Kovács-Simon A, Odendaal L, Molnár E, Giricz Z, Janáky T, van Rooyen J, Csont T, et al. (2008) Lovastatin interferes with the infarct size-limiting effect of ischemic preconditioning and postconditioning in rat hearts. Am J Physiol Heart Circ Physiol 294:H2406-H2409.

Kocsis GF, Sárközy M, Bencsik P, Pipicz M, Varga ZV, Pálóczi J, Csonka C, Ferdinandy $\mathrm{P}$, and Csont T (2012) Preconditioning protects the heart in a prolonged uremic condition. Am J Physiol Heart Circ Physiol 303:H1229-H1236.

Kottenberg E, Musiolik J, Thielmann M, Jakob H, Peters J, and Heusch G (2014a) Interference of propofol with signal transducer and activator of transcription 5 activation and cardioprotection by remote ischemic preconditioning during coronary artery bypass grafting. $J$ Thorac Cardiovasc Surg 147:376-382.

Kottenberg E, Thielmann M, Bergmann L, Heine T, Jakob H, Heusch G, and Peters J (2012) Protection by remote ischemic preconditioning during coronary artery bypass graft surgery with isoflurane but not propofol - a clinical trial. Acta Anaes thesiol Scand 56:30-38.

Kottenberg E, Thielmann M, Kleinbongard P, Frey UH, Heine T, Jakob H, Heusch G, and Peters $J(2014 \mathrm{~b})$ Myocardial protection by remote ischaemic pre-conditioning is abolished in sulphonylurea-treated diabetics undergoing coronary revascularisation. Acta Anaesthesiol Scand 58:453-462.

Kristensen J, Mortensen UM, Schmidt M, Nielsen PH, Nielsen TT, and Maeng M (2009) Lack of cardioprotection from subcutaneously and preischemic administered liraglutide in a closed chest porcine ischemia reperfusion model. BMC Cardiovasc Disord 9:31.

Kubli DA and Gustafsson AB (2012) Mitochondria and mitophagy: the yin and yang of cell death control. Circ Res 111:1208-1221.

Kunuthur SP, Mocanu MM, Hemmings BA, Hausenloy DJ, and Yellon DM (2012) The Akt1 isoform is an essential mediator of ischaemic preconditioning. J Cell Mol Med 16:1739-1749.

Kupai K, Csonka C, Fekete V, Odendaal L, van Rooyen J, Marais W, Csont T, and Ferdinandy P (2009) Cholesterol diet-induced hyperlipidemia impairs the cardioprotective effect of postconditioning: role of peroxynitrite. Am J Physiol Heart Circ Physiol 297:H1729-H1735.

Lacerda L, Opie LH, and Lecour S (2012) Influence of tumour necrosis factor alpha on the outcome of ischaemic postconditioning in the presence of obesity and diabetes. Exp Diabetes Res 2012:502654

Lacerda L, Somers S, Opie LH, and Lecour S (2009) Ischaemic postconditioning protects against reperfusion injury via the SAFE pathway. Cardiovasc Res 84: 201-208.

Lalu MM, Csonka C, Giricz Z, Csont T, Schulz R, and Ferdinandy P (2002) Preconditioning decreases ischemia/reperfusion-induced release and activation of matrix metalloproteinase-2. Biochem Biophys Res Commun 296:937-941.

Lancaster TS, Jefferson SJ, and Korzick DH (2011) Local delivery of a PKC $\varepsilon$ activating peptide limits ischemia reperfusion injury in the aged female rat heart. Am J Physiol Regul Integr Comp Physiol 301:R1242-R1249.

Landim MB, Dourado PM, Casella-Filho A, Chagas AC, and da-Luz PL (2013) High plasma concentrations of asymmetric dimethylarginine inhibit ischemic cardioprotection in hypercholesterolemic rats. Braz J Med Biol Res 46:454-459.

Lange M, Redel A, Lotz C, Smul TM, Blomeyer C, Frank A, Stumpner J, Roewer N, and Kehl F (2009) Desflurane-induced postconditioning is mediated by betaadrenergic signaling: role of beta 1 - and beta 2 -adrenergic receptors, protein kinase $\mathrm{A}$, and calcium/calmodulin-dependent protein kinase II. Anesthesiology 110: 516-528.

Lange M, Smul TM, Blomeyer CA, Redel A, Klotz KN, Roewer N, and Kehl F (2006) Role of the beta1-adrenergic pathway in anesthetic and ischemic preconditioning against myocardial infarction in the rabbit heart in vivo. Anesthesiology 105: 503-510.

Laskey WK, Yoon S, Calzada N, and Ricciardi MJ (2008) Concordant improvements in coronary flow reserve and ST-segment resolution during percutaneous coronary intervention for acute myocardial infarction: a benefit of postconditioning. Catheter Cardiovasc Interv 72:212-220.

Lecour S (2009) Activation of the protective Survivor Activating Factor Enhancement (SAFE) pathway against reperfusion injury: Does it go beyond the RISK pathway? $J$ Mol Cell Cardiol 47:32-40.

Lee JH, Park YH, Byon HJ, Kim HS, Kim CS, and Kim JT (2012) Effect of remote ischaemic preconditioning on ischaemic-reperfusion injury in pulmonary hypertensive infants receiving ventricular septal defect repair. $\mathrm{Br} J$ Anaesth 108: $223-228$.

Lemoine S, Beauchef G, Zhu L, Renard E, Lepage O, Massetti M, Khayat A, Galera P, Gérard JL, and Hanouz JL (2008) Signaling pathways involved in desflurane-induced 
postconditioning in human atrial myocardium in vitro. Anesthesiology 109 1036-1044.

Lemoine S, Durand C, Zhu L, Ivasceau C, Lepage O, Babatasi G, Massetti M, Gérard JL, and Hanouz JL (2010) Desflurane-induced postconditioning of diabetic human right atrial myocardium in vitro. Diabetes Metab 36:21-28.

Lemoine S, Zhu L, Buléon C, Massetti M, Gérard JL, Galera P, and Hanouz JL (2011) Mechanisms involved in the desflurane-induced post-conditioning of isolated human right atria from patients with type 2 diabetes. $\mathrm{Br}$ J Anaesth 107:510-518.

Levey AS and Coresh J (2012) Chronic kidney disease. Lancet 379:165-180.

Li B, Chen R, Huang R, and Luo W (2009) Clinical benefit of cardiac ischemic postconditioning in corrections of tetralogy of Fallot. Interact Cardiovasc Thorac Surg 8:17-21.

$\mathrm{Li} \mathrm{G}$, Chen S, Lu E, and Li Y (1999) Ischemic preconditioning improves preservation with cold blood cardioplegia in valve replacement patients. Eur J Cardiothorac Surg 15:653-657.

Li L, Luo W, Huang L, Zhang W, Gao Y, Jiang H, Zhang C, Long L, and Chen S (2010) Remote perconditioning reduces myocardial injury in adult valve replacement: a randomized controlled trial. J Surg Res 164:e21-e26.

Liem DA, Verdouw PD, Ploeg H, Kazim S, and Duncker DJ (2002) Sites of action of adenosine in interorgan preconditioning of the heart. Am J Physiol Heart Circ Physiol 283:H29-H37.

Lim SY, Davidson SM, Mocanu MM, Yellon DM, and Smith CC (2007) The cardioprotective effect of necrostatin requires the cyclophilin-D component of the mitochondrial permeability transition pore Cardiovasc Drugs Ther 21:467-469.

Ling S, Birnbaum Y, Nanhwan MK, Thomas B, Bajaj M, Li Y, Li Y, and Ye Y (2013) Dickkopf-1 (DKK1) phosphatase and tensin homolog on chromosome 10 (PTEN) crosstalk via microRNA interference in the diabetic heart. Basic Res Cardiol 108: 352.

Liu $\mathrm{H}$ and Moore PG (2010) $\mathrm{K}_{\mathrm{ATP}}$ channel blocker does not abolish the protective effect of $\mathrm{Na} / \mathrm{H}+$ exchange 1 inhibition against ischaemia/reperfusion in aged myocardium. Eur J Anaesthesiol 27:740-746.

Liu TK, Mishra AK, and Ding FX (2011a) [Protective effect of ischemia postconditioning on reperfusion injury in patients with ST-segment elevation acute myocardial infarction]. Zhonghua Xin Xue Guan Bing Za Zhi 39:35-39.

Liu SH, Huo YE, Yin BY, Li XH, and Wang YF (2013) Ischemic postconditioning may increase serum fetuin-A level in patients with acute ST-segment elevation myocardial infarction undergoing percutaneous intervention. Clin Lab 59:59-64

Liu L, Zhu J, Brink PR, Glass PS, and Rebecchi MJ (2011b) Age-associated differences in the inhibition of mitochondrial permeability transition pore opening by cyclosporine A. Acta Anaesthesiol Scand 55:622-630.

Liuni A, Luca MC, Gori T, and Parker JD (2012) Loss of the preconditioning effect of rosuvastatin during sustained therapy: a human in vivo study. Am J Physiol Heart Circ Physiol 302:H153-H158.

Lomivorotov VV, Shmyrev VA, Nepomnyaschih VA, Ponomarev DN, Knyazkova LG, Lomivorotov VN, and Karaskov AM (2012) Remote ischaemic preconditioning does not protect the heart in patients undergoing coronary artery bypass grafting Interact Cardiovasc Thorac Surg 15:18-22.

Lønborg J, Kelbaek H, Vejlstrup N, Jørgensen E, Helqvist S, Saunamäki K, Clemmensen P, Holmvang L, Treiman M, Jensen JS, et al. (2010) Cardioprotective effects of ischemic postconditioning in patients treated with primary percutaneous coronary intervention, evaluated by magnetic resonance. Circ Cardiovasc Interv $\mathbf{3}$ $34-41$

Lønborg J, Vejlstrup N, Kelbæk H, Bøtker HE, Kim WY, Mathiasen AB, Jørgensen E, Helqvist S, Saunamäki K, Clemmensen P, et al. (2012) Exenatide reduces reperfusion injury in patients with ST-segment elevation myocardial infarction. Eur Heart $J$ 33:1491-1499.

Loubeyre C, Morice MC, Lefèvre T, Piéchaud JF, Louvard Y, and Dumas P (2002) A randomized comparison of direct stenting with conventional stent implantation in selected patients with acute myocardial infarction. J Am Coll Cardiol 39:15-21.

Lu E-X, Chen S-X, Yuan M-D, Hu T-H, Zhou H-C, Luo W-J, Li G-H, and Xu L-M (1997) Preconditioning improves myocardial preservation in patients undergoing open heart operations. Ann Thorac Surg 64:1320-1324.

Lucchinetti E, Bestmann L, Feng J, Freidank H, Clanachan AS, Finegan BA, and Zaugg M (2012) Remote ischemic preconditioning applied during isoflurane inhalation provides no benefit to the myocardium of patients undergoing on-pump coronary artery bypass graft surgery: lack of synergy or evidence of antagonism in cardioprotection? Anesthesiology 116:296-310.

Lucchinetti E, Jamnicki M, Fischer G, and Zaugg M (2008) Preconditioning by isoflurane retains its protection against ischemia-reperfusion injury in postinfarct remodeled rat hearts. Anesth Analg 106:17-23.

Ludman A, Venugopal V, Yellon DM, and Hausenloy DJ (2009) Statins and cardioprotection-more than just lipid lowering? Pharmacol Ther 122:30-43.

Ludman AJ, Hausenloy DJ, Babu G, Hasleton J, Venugopal V, Boston-Griffiths E, Yap J, Lawrence D, Hayward M, Kolvekar S, et al. (2011) Failure to recapture cardioprotection with high-dose atorvastatin in coronary artery bypass surgery: a randomised controlled trial. Basic Res Cardiol 106:1387-1395.

Ludman AJ, Yellon DM, and Hausenloy DJ (2010) Cardiac preconditioning for ischaemia: lost in translation. Dis Model Mech 3:35-38.

Luo SJ, Zhou YJ, Shi DM, Ge HL, Wang JL, and Liu RF (2013) Remote ischemic preconditioning reduces myocardial injury in patients undergoing coronary stent implantation. Can J Cardiol 29:1084-1089.

Luo W, Li B, Chen R, Huang R, and Lin G (2008a) Effect of ischemic postconditioning in adult valve replacement. Eur J Cardiothorac Surg 33:203-208.

Luo W, Li B, Lin G, Chen R, and Huang R (2008b) Does cardioplegia leave room for postconditioning in paediatric cardiac surgery? Cardiol Young 18:282-287.

Luo W, Li B, Lin G, and Huang R (2007) Postconditioning in cardiac surgery for tetralogy of Fallot. J Thorac Cardiovasc Surg 133:1373-1374.

Luo W, Zhu M, Huang R, and Zhang Y (2011) A comparison of cardiac postconditioning and remote pre-conditioning in paediatric cardiac surgery. Cardiol Young 21:266-270.
Ma XJ, Zhang XH, Li CM, and Luo M (2006) Effect of postconditioning on coronary blood flow velocity and endothelial function in patients with acute myocardial infarction. Scand Cardiovasc J 40:327-333.

Mahalwar R and Khanna D (2013) Pleiotropic antioxidant potential of rosuvastatin in preventing cardiovascular disorders. Eur $J$ Pharmacol 711:57-62.

Marso SP, Miller T, Rutherford BD, Gibbons RJ, Qureshi M, Kalynych A, Turco M, Schultheiss HP, Mehran R, Krucoff MW, et al. (2007) Comparison of myocardial reperfusion in patients undergoing percutaneous coronary intervention in STsegment elevation acute myocardial infarction with versus without diabetes mellitus (from the EMERALD Trial). Am J Cardiol 100:206-210.

Matsumoto S, Cho S, Tosaka S, Ureshino H, Maekawa T, Hara T, and Sumikawa K (2009) Pharmacological preconditioning in type 2 diabetic rat hearts: the roles of mitochondrial ATP-sensitive potassium channels and the phosphatidylinositol 3kinase-Akt pathway. Cardiovasc Drugs Ther 23:263-270.

Messadi E, Vincent MP, Griol-Charhbili V, Mandet C, Colucci J, Krege JH, Bruneval P, Bouby N, Smithies O, Alhenc-Gelas F, et al. (2010) Genetically determined angiotensin converting enzyme level and myocardial tolerance to ischemia. FASEB $J$ 24:4691-4700.

Mewton N, Croisille P, Gahide G, Rioufol G, Bonnefoy E, Sanchez I, Cung TT, Sportouch C, Angoulvant D, Finet G, et al. (2010) Effect of cyclosporine on left ventricular remodeling after reperfused myocardial infarction. J Am Coll Cardio 55:1200-1205.

Mewton N, Thibault H, Roubille F, Lairez O, Rioufol G, Sportouch C, Sanchez I, Bergerot C, Cung TT, Finet G, et al. (2013) Postconditioning attenuates no-reflow in STEMI patients. Basic Res Cardiol 108:383.

Meybohm P, Renner J, Broch O, Caliebe D, Albrecht M, Cremer J, Haake N, Scholz J, Zacharowski K, and Bein B (2013) Postoperative neurocognitive dysfunction in patients undergoing cardiac surgery after remote ischemic preconditioning: a double-blind randomized controlled pilot study. PLoS ONE 8:e64743.

Mihaylova B, Emberson J, Blackwell L, Keech A, Simes J, Barnes EH, Voysey M, Gray A, Collins R, and Baigent C; Cholesterol Treatment Trialists' (CTT) Collaborators (2012) The effects of lowering LDL cholesterol with statin therapy in people at low risk of vascular disease: meta-analysis of individual data from 27 randomised trials. Lancet 380:581-590.

Miki T, Itoh T, Sunaga D, and Miura T (2012) Effects of diabetes on myocardial infarct size and cardioprotection by preconditioning and postconditioning. Cardiovasc Diabetol 11:67.

Miki T, Miura T, Hotta H, Tanno M, Yano T, Sato T, Terashima Y, Takada A Ishikawa S, and Shimamoto K (2009) Endoplasmic reticulum stress in diabetic hearts abolishes erythropoietin-induced myocardial protection by impairment of phospho-glycogen synthase kinase-3beta-mediated suppression of mitochondrial permeability transition. Diabetes 58:2863-2872.

Mills EJ, O'Regan C, Eyawo O, Wu P, Mills F, Berwanger O, and Briel M (2011) Intensive statin therapy compared with moderate dosing for prevention of cardiovascular events: a meta-analysis of $>40000$ patients. Eur Heart J 32 1409-1415.

Moro L, Pedone C, Mondì A, Nunziata E, and Antonelli Incalzi R (2011) Effect of local and remote ischemic preconditioning on endothelial function in young people and healthy or hypertensive elderly people. Atherosclerosis 219:750-752.

Morrison A, Yan X, Tong C, and Li J (2011) Acute rosiglitazone treatment is cardioprotective against ischemia-reperfusion injury by modulating AMPK, Akt, and JNK signaling in nondiabetic mice. Am J Physiol Heart Circ Physiol 301: H895-H902.

Morrison RR, Tan XL, Ledent C, Mustafa SJ, and Hofmann PA (2007) Targeted deletion of A2A adenosine receptors attenuates the protective effects of myocardial postconditioning. Am J Physiol Heart Circ Physiol 293:H2523-H2529.

Munk K, Andersen NH, Schmidt MR, Nielsen SS, Terkelsen CJ, Sloth E, Bøtker HE Nielsen TT, and Poulsen SH (2010) Remote ischemic conditioning in patients with myocardial infarction treated with primary angioplasty: Impact on left ventricular function assessed by comprehensive echocardiography and gated single-photon emission CT. Circ Cardiovasc Imaging 3:656-662.

Musiolik J, van Caster P, Skyschally A, Boengler K, Gres P, Schulz R, and Heusch G (2010) Reduction of infarct size by gentle reperfusion without activation of reperfusion injury salvage kinases in pigs. Cardiovasc Res 85:110-117.

Nadtochiy SM, Redman E, Rahman I, and Brookes PS (2011a) Lysine deacetylation in ischaemic preconditioning: the role of SIRT1. Cardiovasc Res 89:643-649.

Nadtochiy SM, Yao H, McBurney MW, Gu W, Guarente L, Rahman I, and Brookes PS (2011b) SIRT1-mediated acute cardioprotection. Am J Physiol Heart Circ Physiol 301:H1506-H1512.

Nemlin C, Benhabbouche S, Bopassa JC, Sebbag L, Ovize M, and Ferrera R (2009) Optimal pressure for low pressure controlled reperfusion to efficiently protect ischemic heart: an experimental study in rats. Transplant Proc 41:703-704.

Nissen SE and Wolski K (2007) Effect of rosiglitazone on the risk of myocardial infarction and death from cardiovascular causes. N Engl J Med 356:2457-2471.

Noyan-Ashraf MH, Momen MA, Ban K, Sadi AM, Zhou YQ, Riazi AM, Baggio LL, Henkelman RM, Husain M, and Drucker DJ (2009) GLP-1R agonist liraglutide activates cytoprotective pathways and improves outcomes after experimental myocardial infarction in mice. Diabetes 58:975-983.

Oerlemans MI, Liu J, Arslan F, den Ouden K, van Middelaar BJ, Doevendans PA, and Sluijter JP (2012) Inhibition of RIP1-dependent necrosis prevents adverse cardiac remodeling after myocardial ischemia-reperfusion in vivo. Basic Res Car diol 107:270.

Ogawa K, Ikewaki K, Taniguchi I, Takatsuka H, Mori C, Sasaki H, Okazaki F, Shimizu M, and Mochizuki S (2007) Mitiglinide, a novel oral hypoglycemic agent, preserves the cardioprotective effect of ischemic preconditioning in isolated perfused rat hearts. Int Heart $J$ 48:337-345.

Oikawa M, Yaoita H, Watanabe K, and Maruyama Y (2008) Attenuation of cardioprotective effect by postconditioning in coronary stenosed rat heart and its restoration by carvedilol. Circ J 72:2081-2086. 
Olsen AM, Fosbøl EL, Lindhardsen J, Folke F, Charlot M, Selmer C, Bjerring Olesen J, Lamberts M, Ruwald MH, Køber L, et al. (2012) Long-term cardiovascular risk of nonsteroidal anti-inflammatory drug use according to time passed after first-time myocardial infarction: a nationwide cohort study. Circulation 126:1955-1963.

Ong SB and Hausenloy DJ (2010) Mitochondrial morphology and cardiovascular disease. Cardiovasc Res 88:16-29.

Ong SB, Hall AR, and Hausenloy DJ (2013) Mitochondrial dynamics in cardiovascular health and disease. Antioxid Redox Signal 19:400-414.

Ong SB, Subrayan S, Lim SY, Yellon DM, Davidson SM, and Hausenloy DJ (2010) Inhibiting mitochondrial fission protects the heart against ischemia/reperfusion injury. Circulation 121:2012-2022.

Oosterlinck W, Dresselaers T, Geldhof V, Nevelsteen I, Janssens S, Himmelreich U, and Herijgers P (2013) Diabetes mellitus and the metabolic syndrome do not abolish, but might reduce, the cardioprotective effect of ischemic postconditioning. $J$ Thorac Cardiovasc Surg 145:1595-1602.

Opie LH, Yusuf S, and Kübler W (2000) Current status of safety and efficacy of calcium channel blockers in cardiovascular diseases: a critical analysis based on 100 studies. Prog Cardiovasc Dis 43:171-196.

Osipov RM, Bianchi C, Feng J, Clements RT, Liu Y, Robich MP, Glazer HP, Sodha NR and Sellke FW (2009) Effect of hypercholesterolemia on myocardial necrosis and apoptosis in the setting of ischemia-reperfusion. Circulation 120(11, Suppl)S22-S30.

Ostadal B, Netuka I, Maly J, Besik J, and Ostadalova I (2009) Gender differences in cardiac ischemic injury and protection-experimental aspects. Exp Biol Med (Maywood) 234:1011-1019.

Otto S, Seeber M, Fujita B, Kretzschmar D, Ferrari M, Goebel B, Figulla HR, and Poerner TC (2012) Microembolization and myonecrosis during elective percutaneous coronary interventions in diabetic patients: an intracoronary Doppler ultrasound study with 2-year clinical follow-up. Basic Res Cardiol 107.289.

Ovize M, Baxter GF, Di Lisa F, Ferdinandy P, Garcia-Dorado D, Hausenloy DJ, Heusch G, Vinten-Johansen J, Yellon DM, and Schulz R; Working Group of Cellular Biology of Heart of European Society of Cardiology (2010) Postconditioning and protection from reperfusion injury: where do we stand? Position paper from the Working Group of Cellular Biology of the Heart of the European Society of Cardiology. Cardiovasc Res 87:406-423.

Ovünç K (2000) Effects of glibenclamide, a $\left.\mathrm{K}_{(\mathrm{ATP}}\right)$ channel blocker, on warm-up phenomenon in type II diabetic patients with chronic stable angina pectoris. Clin Cardiol 23:535-539.

Oxman T, Arad M, Klein R, Avazov N, and Rabinowitz B (1997) Limb ischemia preconditions the heart against reperfusion tachyarrhythmia. Am J Physiol 273 H1707-H1712.

Palee S, Weerateerangkul P, Chinda K, Chattipakorn SC, and Chattipakorn N (2013) Mechanisms responsible for beneficial and adverse effects of rosiglitazone in a rat model of acute cardiac ischaemia-reperfusion. Exp Physiol 98:1028-1037.

Pan X, Liu J, Nguyen T, Liu C, Sun J, Teng Y, Fergusson MM, Rovira II, Allen M, Springer DA, et al. (2013) The physiological role of mitochondrial calcium revealed by mice lacking the mitochondrial calcium uniporter. Nat Cell Biol 15:1464-1472.

Papanicolaou KN, Khairallah RJ, Ngoh GA, Chikando A, Luptak I, O'Shea KM, Riley DD, Lugus JJ, Colucci WS, Lederer WJ, et al. (2011) Mitofusin-2 maintains mitochondrial structure and contributes to stress-induced permeability transition in cardiac myocytes. Mol Cell Biol 31:1309-1328.

Papanicolaou KN, Ngoh GA, Dabkowski ER, O'Connell KA, Ribeiro RF Jr, Stanley WC, and Walsh K (2012) Cardiomyocyte deletion of mitofusin-1 leads to mitochondrial fragmentation and improves tolerance to ROS-induced mitochondrial dysfunction and cell death. Am J Physiol Heart Circ Physiol 302:H167-H179.

Pateliya BB, Singh N, and Jaggi AS (2008) Possible role of opioids and KATP channels in neuroprotective effect of postconditioning in mice. Biol Pharm Bull 31:1755-1760.

Pavione MA, Carmona F, de Castro M, and Carlotti AP (2012) Late remote ischemic preconditioning in children undergoing cardiopulmonary bypass: a randomized controlled trial. J Thorac Cardiovasc Surg 144:178-183.

Pêgo-Fernandes PM, Jatene FB, Kwasnicka K, Hueb AC, Moreira LF, Gentil AF, Stolf NAG, and Oliveira SA (2000) Ischemic preconditioning in myocardial revascularization with intermittent aortic cross-clamping. J Card Surg 15:333-338, discussion 339-340.

Pei H, Qu Y, Lu X, Yu Q, Lian K, Liu P, Yan W, Liu J, Ma Y, Liu Y, et al. (2013) Cardiac-derived adiponectin induced by long-term insulin treatment ameliorates myocardial ischemia/reperfusion injury in type 1 diabetic mice via AMPK signaling. Basic Res Cardiol 108:322.

Penna C, Mancardi D, Tullio F, and Pagliaro P (2008) Postconditioning and intermittent bradykinin induced cardioprotection require cyclooxygenase activation and prostacyclin release during reperfusion. Basic Res Cardiol 103:368-377.

Penna C, Tullio F, Moro F, Folino A, Merlino A, and Pagliaro P (2010) Effects of a protocol of ischemic postconditioning and/or captopril in hearts of normotensive and hypertensive rats. Basic Res Cardiol 105:181-192.

Penna C, Tullio F, Perrelli MG, Moro F, Abbadessa G, Piccione F, Carriero V, Racca $\mathrm{S}$, and Pagliaro $\mathrm{P}$ (2011) Ischemia/reperfusion injury is increased and cardioprotection by a postconditioning protocol is lost as cardiac hypertrophy develops in nandrolone treated rats. Basic Res Cardiol 106:409-420.

Penna C, Mancardi D, Rastaldo R, Losano G, and Pagliaro P (2007) Intermittent activation of bradykinin B2 receptors and mitochondrial KATP channels trigger cardiac postconditioning through redox signaling. Cardiovasc Res 75:168-177.

Pepe S, Liaw NY, Hepponstall M, Sheeran FL, Yong MS, d'Udekem Y, Cheung MM and Konstantinov IE (2013) Effect of remote ischemic preconditioning on phosphorylated protein signaling in children undergoing tetralogy of Fallot repair: a randomized controlled trial. J Am Heart Assoc 2:e00095.

Perrault LP, Menasché P, Bel A, de Chaumaray T, Peynet J, Mondry A, Olivero P, Emanoil-Ravier R, and Moalic J-M (1996) Ischemic preconditioning in cardiac surgery: a word of caution. J Thorac Cardiovasc Surg 112:1378-1386.

Philipp S, Yang XM, Cui L, Davis AM, Downey JM, and Cohen MV (2006) Postconditioning protects rabbit hearts through a protein kinase C-adenosine A2b receptor cascade. Cardiovasc Res 70:308-314
Phung OJ, Schwartzman E, Allen RW, Engel SS, and Rajpathak SN (2013) Sulphonylureas and risk of cardiovascular disease: systematic review and metaanalysis. Diabet Med 30:1160-1171.

Piot C, Croisille P, Staat P, Thibault H, Rioufol G, Mewton N, Elbelghiti R, Cung TT, Bonnefoy E, Angoulvant D, et al. (2008) Effect of cyclosporine on reperfusion injury in acute myocardial infarction. $N$ Engl J Med 359:473-481.

Pons S, Martin V, Portal L, Zini R, Morin D, Berdeaux A, and Ghaleh B (2013) Regular treadmill exercise restores cardioprotective signaling pathways in obese mice independently from improvement in associated co-morbidities. $\mathrm{J} \mathrm{Mol} \mathrm{Cell}$ Cardiol 54:82-89.

Potier L, Waeckel L, Vincent MP, Chollet C, Gobeil F Jr, Marre M, Bruneval P, Richer C, Roussel R, Alhenc-Gelas F, et al. (2013) Selective kinin receptor agonists as cardioprotective agents in myocardial ischemia and diabetes. J Pharmacol Exp Ther 346:23-30.

Povlsen JA, Løfgren B, Rasmussen LE, Nielsen JM, Nørregaard R, Kristiansen SB, Bøtker HE, and Nielsen TT (2009) Cardioprotective effect of L-glutamate in obese type 2 diabetic Zucker fatty rats. Clin Exp Pharmacol Physiol 36:892-898.

Prasad A, Gössl M, Hoyt J, Lennon RJ, Polk L, Simari R, Holmes DR Jr, Rihal CS, and Lerman A (2013) Remote ischemic preconditioning immediately before percutaneous coronary intervention does not impact myocardial necrosis, in flammatory response, and circulating endothelial progenitor cell counts: a single center randomized sham controlled trial. Catheter Cardiovasc Interv 81:930-936.

Prunier F, Angoulvant D, Saint Etienne C, Vermes E, Gilard M, Piot C, Roubille F, Elbaz M, Ovize M, Bière L, et al. (2014) The RIPOST-MI study, assessing remote ischemic perconditioning alone or in combination with local ischemic postconditioning in ST-segment elevation myocardial infarction. Basic Res Cardiol 109: 400.

Przyklenk K (2011) Efficacy of cardioprotective 'conditioning' strategies in aging and diabetic cohorts: the co-morbidity conundrum. Drugs Aging 28:331-343.

Przyklenk K, Bauer B, Ovize M, Kloner RA, and Whittaker P (1993) Regional is chemic 'preconditioning' protects remote virgin myocardium from subsequent sustained coronary occlusion. Circulation 87:893-899.

Przyklenk K and Heusch G (2003) Late preconditioning against myocardial stunning. Does aspirin close the "second window" of endogenous cardioprotection? J Am Coll Cardiol 41:1195-1197.

Przyklenk K, Maynard M, Darling CE, and Whittaker P (2008) Aging mouse hearts are refractory to infarct size reduction with post-conditioning. J Am Coll Cardiol 51:1393-1398.

Przyklenk K, Maynard M, Greiner DL, and Whittaker P (2011) Cardioprotection with postconditioning: loss of efficacy in murine models of type-2 and type-1 diabetes. Antioxid Redox Signal 14:781-790.

Rahman IA, Mascaro JG, Steeds RP, Frenneaux MP, Nightingale P, Gosling P, Townsend P, Townend JN, Green D, and Bonser RS (2010) Remote ischemic preconditioning in human coronary artery bypass surgery: from promise to disappointment? Circulation 122(11, Suppl)S53-S59.

Rahmi RM, Uchida AH, Rezende PC, Lima EG, Garzillo CL, Favarato D, Strunz CM, Takiuti M, Girardi P, Hueb W, et al. (2013) Effect of hypoglycemic agents on ischemic preconditioning in patients with type 2 diabetes and symptomatic coronary artery disease. Diabetes Care 36:1654-1659.

Raphael J, Gozal Y, Navot N, and Zuo Z (2010) Hyperglycemia inhibits anestheticinduced postconditioning in the rabbit heart via modulation of phosphatidylinositol-3kinase/Akt and endothelial nitric oxide synthase signaling. J Cardiovasc Pharmacol 55:348-357.

Rassaf T, Ferdinandy P, and Schulz R (2014) Nitrite in organ protection. $\mathrm{Br} J$ Pharmacol 171:1-11.

Redington KL, Disenhouse T, Strantzas SC, Gladstone R, Wei C, Tropak MB, Dai X Manlhiot C, Li J, and Redington AN (2012) Remote cardioprotection by direct peripheral nerve stimulation and topical capsaicin is mediated by circulating humoral factors. Basic Res Cardiol 107:241.

Ren JY, Song JX, Lu MY, and Chen H (2011) Cardioprotection by ischemic postconditioning is lost in isolated perfused heart from diabetic rats: Involvement of transient receptor potential vanilloid 1 , calcitonin gene-related peptide and substance P. Regul Pept 169:49-57.

Rentoukas I, Giannopoulos G, Kaoukis A, Kossyvakis C, Raisakis K, Driva M, Panagopoulou V, Tsarouchas K, Vavetsi S, Pyrgakis V, et al. (2010) Cardioprotective role of remote ischemic periconditioning in primary percutaneous coronary intervention: enhancement by opioid action. JACC Cardiovasc Interv 3:49-55.

Rezkalla SH and Kloner RA (2004) Ischemic preconditioning and preinfarction an gina in the clinical arena. Nat Clin Pract Cardiovasc Med 1:96-102.

Roussel R, Travert F, Pasquet B, Wilson PW, Smith SC Jr, Goto S, Ravaud P, Marre M, Porath A, Bhatt DL, et al. Reduction of Atherothrombosis for Continued Health (REACH) Registry Investigators (2010) Metformin use and mortality among patients with diabetes and atherothrombosis. Arch Intern Med 170:1892-1899.

Sánchez JA, Rodríguez-Sinovas A, Barba I, Miró-Casas E, Fernández-Sanz C, RuizMeana M, Alburquerque-Béjar JJ, and García-Dorado D (2013) Activation of RISK and SAFE pathways is not involved in the effects of $\mathrm{Cx} 43$ deficiency on tolerance to ischemia-reperfusion injury and preconditioning protection. Basic Res Cardiol 108: 351.

Sárközy M, Zvara A, Gyémánt N, Fekete V, Kocsis GF, Pipis J, Szücs G, Csonka C, Puskás LG, Ferdinandy P, et al. (2013) Metabolic syndrome influences cardiac gene expression pattern at the transcript level in male ZDF rats. Cardiovasc Diabetol 12:16.

Sato H, Bolli R, Rokosh GD, Bi Q, Dai S, Shirk G, and Tang XL (2007) The cardioprotection of the late phase of ischemic preconditioning is enhanced by postconditioning via a COX-2-mediated mechanism in conscious rats. Am J Physiol Heart Circ Physiol 293:H2557-H2564.

Sauvé M, Ban K, Momen MA, Zhou YQ, Henkelman RM, Husain M, and Drucker DJ (2010) Genetic deletion or pharmacological inhibition of dipeptidyl peptidase-4 improves cardiovascular outcomes after myocardial infarction in mice. Diabetes 59: 1063-1073. 
Saxena P, Aggarwal S, Misso NL, Passage J, Newman MA, Thompson PJ, d'Udekem Y, Praporski S, and Konstantinov IE (2013) Remote ischaemic preconditioning down-regulates kinin receptor expression in neutrophils of patients undergoing heart surgery. Interact Cardiovasc Thorac Surg 17:653-658.

Schmidt MR, Smerup M, Konstantinov IE, Shimizu M, Li J, Cheung M, White PA, Kristiansen SB, Sorensen K, Dzavik V, et al. (2007) Intermittent peripheral tissue ischemia during coronary ischemia reduces myocardial infarction through a KATPdependent mechanism: first demonstration of remote ischemic perconditioning. Am J Physiol Heart Circ Physiol 292:H1883-H1890.

Schmidt MR, Støttrup NB, Michelsen MM, Contractor H, Sørensen KE, Kharbanda RK, Redington AN, and Bøtker HE (2014) Remote ischemic preconditioning impairs ventricular function and increases infarct size after prolonged ischemia in the isolated neonatal rabbit heart. J Thorac Cardiovasc Surg 147:1049-1055.

Schulz R (2005) Pleiotropic effects of statins: acutely good, but chronically bad? J Am Coll Cardiol 45:1292-1294.

Schulz R, Boengler K, Totzeck A, Luo Y, Garcia-Dorado D, and Heusch G (2007) Connexin 43 in ischemic pre- and postconditioning. Heart Fail Rev 12:261-266.

Schwartz Longacre L, Kloner RA, Arai AE, Baines CP, Bolli R, Braunwald E, Downey J, Gibbons RJ, Gottlieb RA, Heusch G, et al.; National Heart, Lung, and Blood Institute, National Institutes of Health (2011) New horizons in cardioprotection recommendations from the 2010 national heart, lung, and blood institute workshop. Circulation 124:1172-1179.

Scirica BM, Bhatt DL, Braunwald E, Steg PG, Davidson J, Hirshberg B, Ohman P, Frederich R, Wiviott SD, Hoffman EB, et al.; SAVOR-TIMI 53 Steering Committee and Investigators (2013) Saxagliptin and cardiovascular outcomes in patients with type 2 diabetes mellitus. N Engl J Med 369:1317-1326.

Selker HP, Beshansky JR, Sheehan PR, Massaro JM, Griffith JL, D'Agostino RB, Ruthazer R, Atkins JM, Sayah AJ, Levy MK, et al. (2012) Out-of-hospital administration of intravenous glucose-insulin-potassium in patients with suspected acute coronary syndromes: the IMMEDIATE randomized controlled trial. JAMA $\mathbf{3 0 7}$ $1925-1933$

Sharma V, Bell RM, and Yellon DM (2012) Targeting reperfusion injury in acute myocardial infarction: a review of reperfusion injury pharmacotherapy. Expert Opin Pharmacother 13:1153-1175.

Shi W and Vinten-Johansen J (2012) Endogenous cardioprotection by ischaemic postconditioning and remote conditioning. Cardiovasc Res 94:206-216.

Sivaraman V, Hausenloy DJ, Wynne AM, and Yellon DM (2010) Preconditioning the diabetic human myocardium. J Cell Mol Med 14 (6B):1740-1746.

Skyschally A, Gres P, Hoffmann S, Haude M, Erbel R, Schulz R, and Heusch G (2007) Bidirectional role of tumor necrosis factor-alpha in coronary microembolization: progressive contractile dysfunction versus delayed protection against infarction. Circ Res 100:140-146.

Skyschally A, van Caster P, Iliodromitis EK, Schulz R, Kremastinos DT, and Heusch G (2009) Ischemic postconditioning: experimental models and protocol algorithms. Basic Res Cardiol 104:469-483.

Skyschally A, Walter B, and Heusch G (2013) Coronary microembolization during early reperfusion: infarct extension, but protection by ischaemic postconditioning. Eur Heart J 34:3314-3321.

Sloth AD, Schmidt MR, Munk K, Kharbanda RK, Redington AN, Schmidt M, Pedersen L, Sørensen HT, and Bøtker HE; CONDI Investigators (2014) Improved longterm clinical outcomes in patients with ST-elevation myocardial infarction undergoing remote ischaemic conditioning as an adjunct to primary percutaneous coronary intervention. Eur Heart $J$ 35:168-175.

Soetkamp D, Nguyen TT, Menazza S, Hirschhäuser C, Hendgen-Cotta UB, Rassaf T, Schlüter KD, Boengler K, Murphy E, and Schulz R (2014) S-Nitrosation of mitochondrial connexin 43 regulates mitochondrial function. Basic Res Cardiol 109:433.

Somers SJ, Lacerda L, Opie L, and Lecour S (2011) Age, genetic characteristics and number of cycles are critical factors to consider for successful protection of the murine heart with postconditioning. Physiol Res 60:971-974.

Sörensson P, Saleh N, Bouvier F, Böhm F, Settergren M, Caidahl K, Tornvall P, Arheden H, Rydén L, and Pernow J (2010) Effect of postconditioning on infarct size in patients with ST elevation myocardial infarction. Heart 96:1710-1715.

Staat P, Rioufol G, Piot C, Cottin Y, Cung TT, L'Huillier I, Aupetit J-F, Bonnefoy E, Finet G, André-Fouët X, et al. (2005) Postconditioning the human heart. Circula tion 112:2143-2148.

Stasch JP, Pacher P, and Evgenov OV (2011) Soluble guanylate cyclase as an emerging therapeutic target in cardiopulmonary disease. Circulation 123: $2263-2273$

Su H, Yuan Y, Wang XM, Lau WB, Wang Y, Wang X, Gao E, Koch WJ, and Ma XL (2013) Inhibition of CTRP9, a novel and cardiac-abundantly expressed cell survival molecule, by $\mathrm{TNF} \alpha$-initiated oxidative signaling contributes to exacerbated cardiac injury in diabetic mice. Basic Res Cardiol 108:315.

Suematsu Y, Anttila V, Takamoto S, and del Nido P (2004) Cardioprotection afforded by ischemic preconditioning interferes with chronic beta-blocker treatment. Scand Cardiovasc $J$ 38.293-299.

Szilvassy Z, Ferdinandy P, Szilvassy J, Nagy I, Karcsu S, Lonovics J, Dux L, and Koltai M (1995) The loss of pacing-induced preconditioning in atherosclerotic rabbits: role of hypercholesterolaemia. J Mol Cell Cardiol 27:2559-2569.

Szmagala P, Morawski W, Krejca M, Gburek T, and Bochenek A (1998) Evaluation of perioperative myocardial tissue damage in ischemically preconditioned human heart during aorto coronary bypass surgery. J Cardiovasc Surg (Torino) 39 791-795.

Szucs G, Bester DJ, Kupai K, Csont T, Csonka C, Esterhuyse AJ, Ferdinandy P, and Van Rooyen J (2011) Dietary red palm oil supplementation decreases infarct size in cholesterol fed rats. Lipids Health Dis 10:103-110.

Szűcs G, Murlasits Z, Török S, Kocsis GF, Pálóczi J, Görbe A, Csont T, Csonka C, and Ferdinandy P (2013) Cardioprotection by farnesol: role of the mevalonate pathway. Cardiovasc Drugs Ther 27:269-277.

Tai W, Shi E, Yan L, Jiang X, Ma H, and Ai C (2012) Diabetes abolishes the cardioprotection induced by sevoflurane postconditioning in the rat heart in vivo: roles of glycogen synthase kinase-3 3 and its upstream pathways. J Surg Res 178: 96-104.

Tarantini G, Favaretto E, Marra MP, Frigo AC, Napodano M, Cacciavillani L, Giovagnoni A, Renda P, De Biasio V, Plebani M, et al. (2012) Postconditioning during coronary angioplasty in acute myocardial infarction: the POST-AMI trial. Int $J$ Cardiol 162:33-38.

Teoh LK, Grant R, Hulf JA, Pugsley WB, and Yellon DM (2002a) A comparison between ischemic preconditioning, intermittent cross-clamp fibrillation and cold crystalloid cardioplegia for myocardial protection during coronary artery bypass graft surgery. Cardiovasc Surg 10:251-255.

Teoh LKK, Grant R, Hulf JA, Pugsley WB, and Yellon DM (2002b) The effect of preconditioning (ischemic and pharmacological) on myocardial necrosis following coronary artery bypass graft surgery. Cardiovasc Res 53:175-180.

Thibault H, Piot C, Staat P, Bontemps L, Sportouch C, Rioufol G, Cung TT, Bonnefoy E, Angoulvant D, Aupetit JF, et al. (2008) Long-term benefit of postconditioning. Circulation 117:1037-1044.

Thielmann M, Kottenberg E, Boengler K, Raffelsieper C, Neuhaeuser M, Peters J, Jakob H, and Heusch G (2010) Remote ischemic preconditioning reduces myocardial injury after coronary artery bypass surgery with crystalloid cardioplegic arrest. Basic Res Cardiol 105:657-664.

Thielmann M, Kottenberg E, Kleinbongard P, Wendt D, Gedik N, Pasa S, Price V, Tsagakis K, Neuhäuser M, Peters J, et al. (2013) Cardioprotective and prognostic effects of remote ischaemic preconditioning in patients undergoing coronary artery bypass surgery: a single-centre randomised, double-blind, controlled trial. Lancet 382:597-604.

Thuny F, Lairez O, Roubille F, Mewton N, Rioufol G, Sportouch C, Sanchez I, Bergerot C, Korzic H, Cung TT, et al. (2012) Post-conditioning reduces infarct size and edema in patients with ST-segment elevation myocardial infarction. J Am Coll Cardiol 59: $2175-2181$

Timmers L, Henriques JP, de Kleijn DP, Devries JH, Kemperman H, Steendijk P, Verlaan CW, Kerver M, Piek JJ, Doevendans PA, et al. (2009) Exenatide reduces infarct size and improves cardiac function in a porcine model of ischemia and reperfusion injury. J Am Coll Cardiol 53:501-510.

Tosaka S, Tosaka R, Matsumoto S, Maekawa T, Cho S, and Sumikawa K (2011) Roles of cyclooxygenase 2 in sevoflurane- and olprinone-induced early phase of preconditioning and postconditioning against myocardial infarction in rat hearts. $J$ Cardiovasc Pharmacol Ther 16:72-78.

Ugata Y, Nakamura T, Taniguchi Y, Ako J, and Momomura S (2012) Effect of postconditioning in patients with ST-elevation acute myocardial infarction. Cardiovasc Interv Ther 27:14-18.

van den Munckhof I, Riksen N, Seeger JP, Schreuder TH, Borm GF, Eijsvogels TM, Hopman MT, Rongen GA, and Thijssen DH (2013) Aging attenuates the protective effect of ischemic preconditioning against endothelial ischemia-reperfusion injury in humans. Am J Physiol Heart Circ Physiol 304:H1727-H1732.

Varga ZV, Kupai K, Szücs G, Gáspár R, Pálóczi J, Faragó N, Zvara A, Puskás LG, Rázga Z, Tiszlavicz L, et al. (2013) MicroRNA-25-dependent up-regulation of NADPH oxidase 4 (NOX4) mediates hypercholesterolemia-induced oxidative/nitrative stress and subsequent dysfunction in the heart. J Mol Cell Cardiol 62:111-121.

Venugopal V, Hausenloy DJ, Ludman A, Di Salvo C, Kolvekar S, Yap J, Lawrence D, Bognolo J, and Yellon DM (2009) Remote ischaemic preconditioning reduces myocardial injury in patients undergoing cardiac surgery with cold-blood cardioplegia: a randomised controlled trial. Heart 95:1567-1571.

Vessey DA, Kelley M, Li L, and Huang Y (2009) Sphingosine protects aging hearts from ischemia/reperfusion injury: Superiority to sphingosine 1-phosphate and ischemic pre- and post-conditioning. Oxid Med Cell Longev 2:146-151.

Vessey DA, Li L, Kelley M, and Karliner JS (2008a) Combined sphingosine, S1P and ischemic postconditioning rescue the heart after protracted ischemia. Biochem Biophys Res Commun 375:425-429.

Vessey DA, Li L, Kelley M, Zhang J, and Karliner JS (2008b) Sphingosine can preand post-condition heart and utilizes a different mechanism from sphingosine 1 phosphate. J Biochem Mol Toxicol 22:113-118.

Vinokur V, Berenshtein E, Bulvik B, Grinberg L, Eliashar R, and Chevion M (2013) The bitter fate of the sweet heart: impairment of iron homeostasis in diabetic heart leads to failure in myocardial protection by preconditioning. PLOS ONE 8:e62948.

Vinten-Johansen J and Shi W (2013) The science and clinical translation of remote postconditioning. J Cardiovasc Med (Hagerstown) 14:206-213.

Wagner C, Ebner B, Tillack D, Strasser RH, and Weinbrenner C (2013) Cardioprotection by ischemic postconditioning is abrogated in hypertrophied myocardium of spontaneously hypertensive rats. J Cardiovasc Pharmacol 61:35-41.

Wagner C, Kloeting I, Strasser RH, and Weinbrenner C (2008) Cardioprotection by postconditioning is lost in WOKW rats with metabolic syndrome: role of glycogen synthase kinase 3beta. J Cardiovasc Pharmacol 52:430-437.

Wagner R, Piler P, Bedanova H, Adamek P, Grodecka L, and Freiberger T (2010) Myocardial injury is decreased by late remote ischaemic preconditioning and aggravated by tramadol in patients undergoing cardiac surgery: a randomised controlled trial. Interact Cardiovasc Thorac Surg 11:758-762.

Wallbridge DR, Schulz R, Braun C, Post H, and Heusch G (1996) No attenuation of ischaemic preconditioning by the calcium antagonist nisoldipine. $\mathrm{J} \mathrm{Mol} \mathrm{Cell} \mathrm{Car-}$ diol 28:1801-1810.

Walsh SR, Tang TY, Kullar P, Jenkins DP, Dutka DP, and Gaunt ME (2008) Ischaemic preconditioning during cardiac surgery: systematic review and metaanalysis of perioperative outcomes in randomised clinical trials. Eur J Cardiothorac Surg 34:985-994.

Wang B, Raedschelders K, Shravah J, Hui Y, Safaei HG, Chen DD, Cook RC, Frade G, Au CL, and Ansley DM (2011b) Differences in myocardial PTEN expression and Akt signalling in type 2 diabetic and nondiabetic patients undergoing coronary bypass surgery. Clin Endocrinol (Oxf) 74:705-713.

Wang JX, Jiao JQ, Li Q, Long B, Wang K, Liu JP, Li YR, and Li PF (2011a) miR-499 regulates mitochondrial dynamics by targeting calcineurin and dynamin-related protein-1. Nat Med 17:71-78. 
Wang Z, Jiang H, Chen S, Du F, and Wang X (2012) The mitochondrial phosphatase PGAM5 functions at the convergence point of multiple necrotic death pathways. Cell 148:228-243.

Watanabe K, Yaoita H, Ogawa K, Oikawa M, Maehara K, and Maruyama Y (2006) Attenuated cardioprotection by ischemic preconditioning in coronary stenosed heart and its restoration by carvedilol. Cardiovasc Res 71:537-547.

Wei M, Xin P, Li S, Tao J, Li Y, Li J, Liu M, Li J, Zhu W, and Redington AN (2011) Repeated remote ischemic postconditioning protects against adverse left ventricular remodeling and improves survival in a rat model of myocardial infarction. Circ Res 108:1220-1225.

White WB, Cannon CP, Heller SR, Nissen SE, Bergenstal RM, Bakris GL, Perez AT, Fleck PR, Mehta CR, Kupfer S, et al.; EXAMINE Investigators (2013) Alogliptin after acute coronary syndrome in patients with type 2 diabetes. $N$ Engl J Med $\mathbf{3 6 9}$ $1327-1335$

Whittington HJ, Babu GG, Mocanu MM, Yellon DM, and Hausenloy DJ (2012) The diabetic heart: too sweet for its own good? Cardiol Res Pract 2012:845698.

Whittington HJ, Hall AR, McLaughlin CP, Hausenloy DJ, Yellon DM, and Mocanu MM (2013a) Chronic metformin associated cardioprotection against infarction: not just a glucose lowering phenomenon. Cardiovasc Drugs Ther 27:5-16.

Whittington HJ, Harding I, Stephenson CI, Bell R, Hausenloy DJ, Mocanu MM, and Yellon DM (2013b) Cardioprotection in the aging, diabetic heart: the loss of protective Akt signalling. Cardiovasc Res 99:694-704.

Wojtovich AP, Nadtochiy SM, Brookes PS, and Nehrke K (2012) Ischemic preconditioning: the role of mitochondria and aging. Exp Gerontol 47:1-7.

Woo JS, Kim W, Ha S.J, Kim JB, Kim S.J, Kim WS, Seon HJ, and Kim KS (2013) Cardioprotective effects of exenatide in patients with ST-segment-elevation myocardial infarction undergoing primary percutaneous coronary intervention: results of exenatide myocardial protection in revascularization study. Arterioscler Thromb Vasc Biol 33:2252-2260.

Wu GT, Wang L, Li J, and Zhu WZ (2007) Effects of glibenclamide, glimepiride, and gliclazide on ischemic preconditioning in rat heart. Chin Med Sci J 22:162-168.

Wu N, Zhang X, Guan Y, Shu W, Jia P, and Jia D (2014) Hypercholesterolemia abrogates the cardioprotection of ischemic postconditioning in isolated rat heart: roles of glycogen synthase kinase- $3 \beta$ and the mitochondrial permeability transition pore. Cell Biochem Biophys 69:123-130.

Wu Q, Gui P, Wu J, Ding D, Purusram G, Dong N, and Yao S (2011a) Effect of limb ischemic preconditioning on myocardial injury in patients undergoing mitral valve replacement surgery. -A randomized controlled trial-. Circ $J$ 75:1885-1889.

Wu Y, Xia ZY, Dou J, Zhang L, Xu JJ, Zhao B, Lei S, and Liu HM (2011b) Protective effect of ginsenoside Rb1 against myocardial ischemia/reperfusion injury in streptozotocin-induced diabetic rats. Mol Biol Rep 38:4327-4335.

Wu ZK, Tarkka MR, Eloranta J, Pehkonen E, Kaukinen L, Honkonen EL, and Kaukinen S (2001) Effect of ischemic preconditioning on myocardial protection in coronary artery bypass graft patients : can the free radicals act as a trigger for ischemic preconditioning? Chest 119:1061-1068.

Xi L, Das A, Zhao ZQ, Merino VF, Bader M, and Kukreja RC (2008) Loss of myocardial ischemic postconditioning in adenosine $\mathrm{A}_{1}$ and bradykinin $\mathrm{B}_{2}$ receptors gene knockout mice. Circulation 118(14, Suppl)S32-S37.

Xie JJ, Liao XL, Chen WG, Huang DD, Chang FJ, Chen W, Luo ZL, Wang ZP, and Ou JS (2012) Remote ischaemic preconditioning reduces myocardial injury in patients undergoing heart valve surgery: randomised controlled trial. Heart 98:384-388.

Xu X, Zhou Y, Luo S, Zhang W, Zhao Y, Yu M, Ma Q, Gao F, Shen H, and Zhang J (2014) Effect of remote ischemic preconditioning in the elderly patients with coronary artery disease with diabetes mellitus undergoing elective drug-eluting stent implantation. Angiology 65:660-666.

Xu Y, Ma LL, Zhou C, Zhang FJ, Kong FJ, Wang WN, Qian LB, Wang CC, Liu XB, Yan M, et al. (2013) Hypercholesterolemic myocardium is vulnerable to ischemiareperfusion injury and refractory to sevoflurane-induced protection. PLOS ONE 8 e76652.

Xue F, Yang X, Zhang B, Zhao C, Song J, Jiang T, and Jiang W (2010) Postconditioning the human heart in percutaneous coronary intervention. Clin Cardiol 33:439-444.

Yadav HN, Singh M, and Sharma PL (2010a) Involvement of GSK-3 $\beta$ in attenuation of the cardioprotective effect of ischemic preconditioning in diabetic rat heart. Mol Cell Biochem 343:75-81.

Yadav HN, Singh M, and Sharma PL (2010b) Modulation of the cardioprotective effect of ischemic preconditioning in hyperlipidaemic rat heart. Eur J Pharmacol 643:78-83.

Yadav HN, Singh M, and Sharma PL (2012) Pharmacological inhibition of GSK-3 $\beta$ produces late phase of cardioprotection in hyperlipidemic rat: possible involvement of HSP 72. Mol Cell Biochem 369:227-233.

Yan W, Zhang H, Liu P, Wang H, Liu J, Gao C, Liu Y, Lian K, Yang L, Sun L, et al. (2013) Impaired mitochondrial biogenesis due to dysfunctional adiponectin-AMPK PGC-1 $\alpha$ signaling contributing to increased vulnerability in diabetic heart. Basic Res Cardiol 108:329.

Yang C, Talukder MA, Varadharaj S, Velayutham M, and Zweier JL (2013) Early ischaemic preconditioning requires Akt- and PKA-mediated activation of eNOS via serine1176 phosphorylation. Cardiovasc Res 97:33-43.

Yang XC, Liu Y, Wang LF, Cui L, Wang T, Ge YG, Wang HS, Li WM, Xu L, Ni ZH, et al. (2007) Reduction in myocardial infarct size by postconditioning in patients after percutaneous coronary intervention. J Invasive Cardiol 19:424-430.

Ye Y, Keyes KT, Zhang C, Perez-Polo JR, Lin Y, and Birnbaum Y (2010a) The myocardial infarct size-limiting effect of sitagliptin is PKA-dependent, whereas the protective effect of pioglitazone is partially dependent on PKA. Am J Physiol Heart Circ Physiol 298:H1454-H1465.

Ye Y, Lin Y, Manickavasagam S, Perez-Polo JR, Tieu BC, and Birnbaum Y (2008) Pioglitazone protects the myocardium against ischemia-reperfusion injury in eNOS and iNOS knockout mice. Am J Physiol Heart Circ Physiol 295 $\mathrm{H} 2436-\mathrm{H} 2446$.

Ye Y, Long B, Qian J, Perez-Polo JR, and Birnbaum Y (2010b) Dipyridamole with low-dose aspirin augments the infarct size-limiting effects of simvastatin. Cardiovasc Drugs Ther 24:391-399.

Ye Y, Perez-Polo JR, Aguilar D, and Birnbaum Y (2011) The potential effects of antidiabetic medications on myocardial ischemia-reperfusion injury. Basic Res Cardiol 106:925-952.

Yellon DM and Hausenloy DJ (2007) Myocardial reperfusion injury. $N$ Engl J Med 357:1121-1135.

Yin M, Silljé HH, Meissner M, van Gilst WH, and de Boer RA (2011) Early and late effects of the DPP-4 inhibitor vildagliptin in a rat model of post-myocardial infarction heart failure. Cardiovasc Diabetol 10:85.

Yin Z, Gao H, Wang H, Li L, Di C, Luan R, and Tao L (2009) Ischaemic postconditioning protects both adult and aged Sprague-Dawley rat heart from ischaemia-reperfusion injury through the phosphatidylinositol 3-kinase-AKT and glycogen synthase kinase-3beta pathways. Clin Exp Pharmacol Physiol 36: $756-763$.

Young PJ, Dalley P, Garden A, Horrocks C, La Flamme A, Mahon B, Miller J, Pilcher J, Weatherall M, Williams J, et al. (2012) A pilot study investigating the effects of remote ischemic preconditioning in high-risk cardiac surgery using a randomised controlled double-blind protocol. Basic Res Cardiol 107:256.

Yu S, Katoh T, Okada H, Makino H, Mimuro S, and Sato S (2010) Landiolol does not enhance the effect of ischemic preconditioning in isolated rat hearts. J Anesth 24 $208-214$.

Zatta AJ, Kin H, Yoshishige D, Jiang R, Wang N, Reeves JG, Mykytenko J, Guyton RA, Zhao ZQ, Caffrey JL, et al. (2008) Evidence that cardioprotection by postconditioning involves preservation of myocardial opioid content and selective opioid receptor activation. Am J Physiol Heart Circ Physiol 294:H1444-H1451.

Zhang FJ, Ma LL, Wang WN, Qian LB, Yang MJ, Yu J, Chen G, Yu LN, and Yan M (2012) Hypercholesterolemia abrogates sevoflurane-induced delayed preconditioning against myocardial infarct in rats by alteration of nitric oxide synthase signaling. Shock 37:485-491.

Zhao H, Wang Y, Wu Y, Li X, Yang G, Ma X, Zhao R, and Liu H (2009a) Hyperlipidemia does not prevent the cardioprotection by postconditioning against myocardial ischemia/reperfusion injury and the involvement of hypoxia inducible factor-1alpha upregulation. Acta Biochim Biophys Sin (Shanghai) 41:745-753.

Zhao WS, Xu L, Wang LF, Zhang L, Zhang ZY, Liu Y, Liu XL, Yang XC, Cui L, and Zhang L (2009b) A 60-s postconditioning protocol by percutaneous coronary intervention inhibits myocardial apoptosis in patients with acute myocardial infarction. Apoptosis 14:1204-1211.

Zhao ZQ, Corvera JS, Halkos ME, Kerendi F, Wang NP, Guyton RA, and VintenJohansen J (2003) Inhibition of myocardial injury by ischemic postconditioning during reperfusion: comparison with ischemic preconditioning. Am J Physiol Heart Circ Physiol 285:H579-H588.

Zhong C, Fleming N, Lu X, Moore P, and Liu H (2012) Age-associated differences in gene expression in response to delayed anesthetic preconditioning. Age (Dordr) 34: $1459-1472$

Zhou C, Liu Y, Yao Y, Zhou S, Fang N, Wang W, and Li L (2013) $\beta$-blockers and volatile anesthetics may attenuate cardioprotection by remote preconditioning in adult cardiac surgery: a meta-analysis of 15 randomized trials. J Cardiothorac Vasc Anesth 27:305-311.

Zhou C, Yao Y, Zheng Z, Gong J, Wang W, Hu S, and Li L (2012) Stenting technique, gender, and age are associated with cardioprotection by ischaemic postconditioning in primary coronary intervention: a systematic review of 10 randomized trials. Eur Heart $J$ 33:3070-3077.

Zhou W, Zeng D, Chen R, Liu J, Yang G, Liu P, and Zhou X (2010) Limb ischemic preconditioning reduces heart and lung injury after an open heart operation in infants. Pediatr Cardiol 31:22-29.

Zhu J, Rebecchi MJ, Glass PS, Brink PR, and Liu L (2011a) Cardioprotection of the aged rat heart by GSK-3beta inhibitor is attenuated: age-related changes in mitochondrial permeability transition pore modulation. Am J Physiol Heart Circ Physiol 300:H922-H930.

Zhu J, Rebecchi MJ, Glass PS, Brink PR, and Liu L (2013a) Interactions of GSK-3 $\beta$ with mitochondrial permeability transition pore modulators during preconditioning: age-associated differences. J Gerontol A Biol Sci Med Sci 68:395-403.

Zhu J, Rebecchi MJ, Tan M, Glass PS, Brink PR, and Liu L (2010) Age-associated differences in activation of Akt/GSK-3beta signaling pathways and inhibition of mitochondrial permeability transition pore opening in the rat heart. $J$ Gerontol $A$ Biol Sci Med Sci 65:611-619.

Zhu J, Rebecchi MJ, Wang Q, Glass PS, Brink PR, and Liu L (2013b) Chronic Tempol treatment restores pharmacological preconditioning in the senescent rat heart. Am J Physiol Heart Circ Physiol 304:H649-H659.

Zhu XH, Yuan HJ, Wu YN, Kang Y, Jiao JJ, Gao WZ, Liu YX, Lou JS, and Xia Z (2011b) Non-invasive limb ischemic pre-conditioning reduces oxidative stress and attenuates myocardium ischemia-reperfusion injury in diabetic rats. Free Radic Res 45:201-210. 